\title{
SECURITY CONSTRAINED STOCHASTIC POWER SYSTEM SCHEDULING ALGORITHMS - WITH WIND ELECTRIC GENERATORS
}

\author{
by
}

\section{Peng $\mathrm{Yu}$}

Master of Science in Engineering, University of New Brunswick, Canada, 2008

Bachelor of Engineering, Shandong University of Technology, China, 2000

\author{
A dissertation \\ presented to Ryerson University \\ in partial fulfillment of the \\ requirements for the degree of \\ Doctor of Philosophy \\ in the Program of
}

Electrical and Computer Engineering

Toronto, Ontario, Canada, 2013

(C) Peng Yu, 2013 


\section{AUTHOR'S DECLARATION}

I hereby declare that I am the sole author of this dissertation. This is a true copy of the dissertation, including any required final revisions, as accepted by my examiners.

I authorize Ryerson University to lend this dissertation to other institutions or individuals for the purpose of scholarly research.

I further authorize Ryerson University to reproduce this dissertation by photocopying or by other means, in total or in part, at the request of other institutions or individuals for the purpose of scholarly research.

I understand that my dissertation may be made electronically available to the public. 


\title{
Abstract
}

\section{Security Constrained Stochastic Power System Scheduling Algorithms - with Wind Electric Generators}

\author{
Peng Yu \\ Doctor of Philosophy \\ Electrical and Computer Engineering \\ Ryerson University, 2013
}

Power systems worldwide are embracing diverse supply mixes that incorporate a significant portion from renewables such as wind and solar energy. Wind energy is characterised by reliable equipment, but with an output that is uncertain and intermittent. In addition to equipment unreliability (system N-1 criterion), output uncertainties of wind electric generators (WEGs) introduce risk into daily power system schedules. This risk from the uncertainty of output from WEGs can be quantified as expected energy not served (EENS). Furthermore, the introduction of new forms of generation changes the methods of operating transmission systems, further necessitating the use of transmission security constraints in power systems optimization algorithms.

This dissertation explores new approaches to stochastically model the real power output of WEGs and to efficiently tackle AC transmission system security constraints for power system 
optimization algorithms such as optimal power flow (OPF) and day-ahead unit commitment (UC).

Usually, normal probabilistic distribution is used to model uncertainty in short-term wind power output forecast and compute EENS. In this dissertation, a new triangular approximate distribution (TAD) model is proposed which is a linear approximation of normal probabilistic distribution to model short-term wind power output forecast and compute EENS. This TAD model is used to formulate a practical risk-constrained fast OPF for transmission systems to simultaneously minimize: 1) risk due to uncertainties in power output from WEGs, and 2) the total operating cost.

The integration of new energy resources causes transmission systems to operate in new, challenging, and often unforeseen operating states. Thus, it is imperative that UC algorithms incorporate AC transmission system security constraints and stochastically model output of WEGs to ensure reliable operation of transmission systems. As a first step, a successive mixed integer linear programming (MILP) method is proposed for AC transmission system security constrained unit commitment (SCUC) challenge. Fuzzy sets theory is used to model infeasible constraints in this MILP formulation.

As a next step, the TAD model of WEGs is integrated into the MILP formulation of SCUC to create a fast security and risk constrained probabilistic UC algorithm. The two UC algorithms are tested on large systems. 


\section{List of Publications}

\section{Publications arising from the PhD work:}

\section{$\underline{\text { Journal Publications }}$}

1. Yu, P., \& Venkatesh, B. (2013). Fuzzy security constraints for unit commitment with outages. IET Generation, Transmission \& Distribution, Accepted for printing.

2. Yu, P., \& Venkatesh, B. (2012). A practical real-time OPF method using new triangular approximate model of wind electric generators. IEEE Transaction on Power Systems, 27(4), 2036-2046.

\section{Conference Publications and Presentations}

1. Yu, P., \& Venkatesh, B. (2012). Triangular approximate distribution model of wind electric generators. The 10th International Power and Energy Conference, Dec. 2012.

2. Yu, P., \& Venkatesh, B. (2012). Unit commitment challenges. Modeling and Optimization: Theory and Applications 2012, Jul. 2012.

3. Yu, P., \& Venkatesh, B. (2012). Successive fuzzy MILP method for AC securityconstrained unit commitment. CORS-MOPGP 2012 International Joint Conference, Jun. 2012. 


\section{$\underline{\text { Articles Submitted to refereed journals }}$}

1. Yu, P., \& Venkatesh, B. (2012). Fast security and risk constrained unit commitment method using TAD model of wind generators. IET Generation, Transmission \& Distribution, under review.

Previous publications arising from the MASc work:

1. Venkatesh, B., Yu, P., \& Gooi, H. B., et al. (2008). Fuzzy MILP unit commitment incorporating wind generators. IEEE Transaction on Power Systems, 23(4), 1738-1746.

2. Choling, D., Yu, P., \& Venkatesh, B. (2009). Effects of security constraints on unit commitment with wind generators. 2009 Power \& Energy Society General Meeting, IEEE, pp. 1-6, Jul. 2009. 


\section{ACKNOWLEDGMENTS}

First and foremost, I would like to express my sincere gratitude to my supervisor, Dr. Bala Venkatesh, for being a mentor, a friend, and a source of inspiration throughout my studies. Without his guidance and direction, I may not have been able to complete this dissertation and achieve a high standard of academic excellence.

I am very grateful to Dr. Daniel Cheng for his valuable comments and suggestions. I would also like to thank Reza Ghaffari, Dr. Chandrabhanu Opathella, and all present and past colleagues in the Power \& Energy Analysis Research (PEAR) laboratory at Ryerson University for their support and encouragement.

I would also like to acknowledge the support that I received from the faculty and staff in the Department of Electrical and Computer Engineering at Ryerson University in Toronto.

Lastly, I would like to thank my parents, wife, and son for their patience, endless love, unwavering support, and encouragement. 


\section{DEDICATION}

To my wife, Fei Yu, and son, James Binglin Yu 


\section{TABLE OF CONTENTS}

AUTHOR'S DECLARATION ..................................................................................................... ii

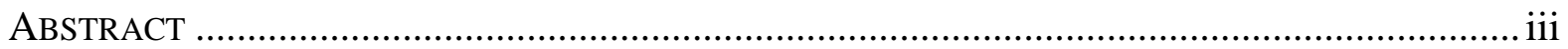

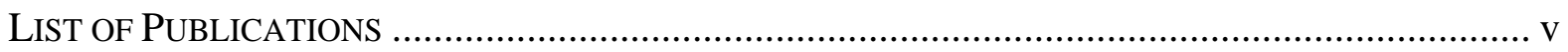

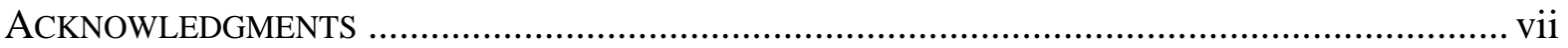

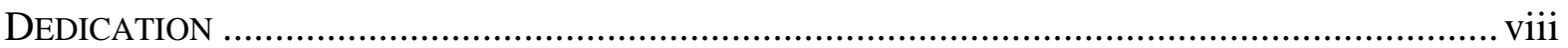

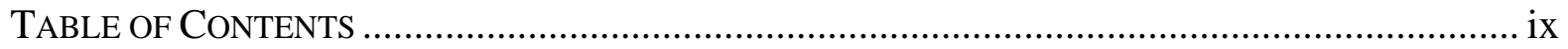

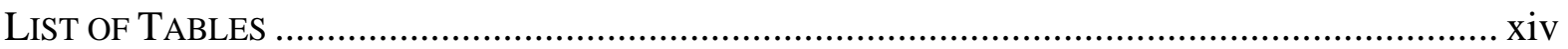

LIST OF FIGURES ..................................................................................................

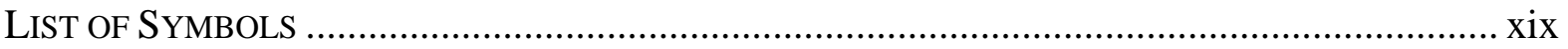

CHAPTER 1 INTRODUCTION....................................................................................... 1

1.1 Probabilistic Modeling of Power Output of Wind Electric Generators .................... 4

1.1.1 Normal Probabilistic Distribution.............................................................. 5

1.1.2 Expected Energy Not Served ............................................................... 6

1.2 Challenges in probabilistic modeling of wind electric generators ........................ 8

1.3 Review of Probabilistic Optimal Power Flow ................................................... 9

1.4 Review of Probabilistic Unit Commitment ...................................................... 12 
1.5 Review of Security Constrained Unit Commitment

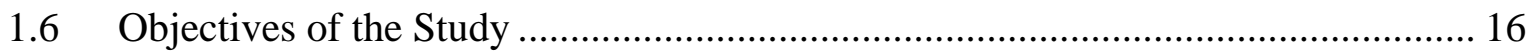

1.7 Chapter-wise Outline of the Dissertation ........................................................... 17

1.8 Chapter Summary.................................................................................. 18

Chapter 2 Triangular ApProximate Distribution Model OF WEGs .......................... 20

2.1 EENS of WEGs using Normal Distribution............................................... 21

2.2 Triangular Approximate Distribution versus Normal Distribution........................ 22

2.3 Probability calculation using the TAD model ................................................... 24

$2.4 \quad$ Estimating EENS using the TAD model ....................................................... 28

2.5 Influence of Wind Uncertainty on EENS value ................................................ 29

2.6 Case study on effects of standard deviation ..................................................... 30

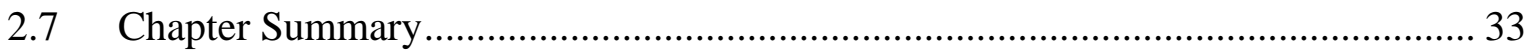

Chapter 3 A Practical FAst Probabilistic OPF MEthod USing the TAD MODEL ......... 34

3.1 Problem formulation of fast Optimal Power Flow............................................ 35

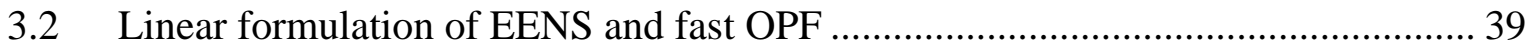

3.2.1 Linearized function of EENS using the TAD model ................................... 39

3.2.2 Complete formulation of fast probabilistic OPF with the TAD model ........... 40

3.3 Algorithm of fast OPF with the TAD model of WEGs...................................... 43

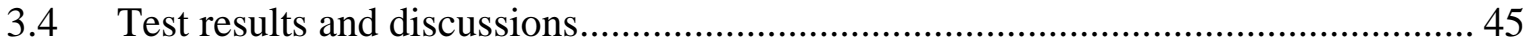

3.4.1 Modified IEEE 30-bus System .............................................................. 45 
3.4.2 IEEE 118-bus System ................................................................................. 51

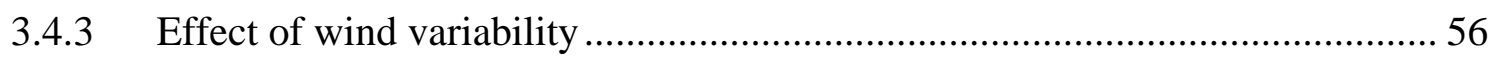

3.5 Chapter Summary ………………………………………………………….... 57

CHAPTER 4 SUCCESSIVE FUZZY MILP METHOD FOR AC SECURITY CONSTRAINED UNIT

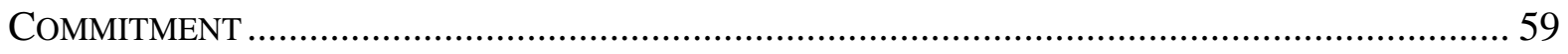

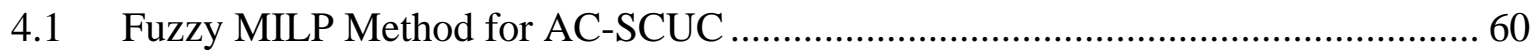

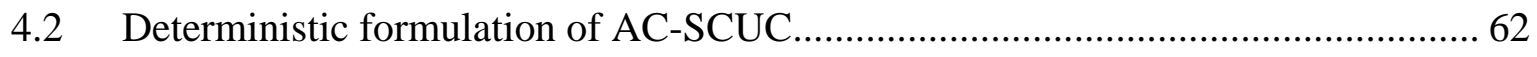

4.3 Linearization (MILP formulation) of AC-SCUC ....................................................... 67

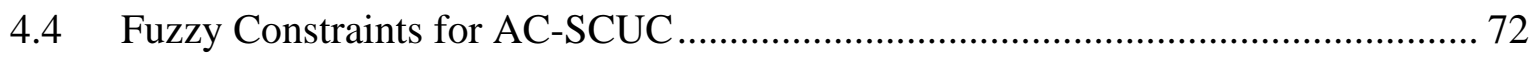

4.4.1 Fuzzy set of objective function ..................................................................... 72

4.4.2 Fuzzy set of Line Flow Limit Constraint ........................................................ 73

4.4.3 Fuzzy sets of Voltage Limit Constraint ............................................................ 74

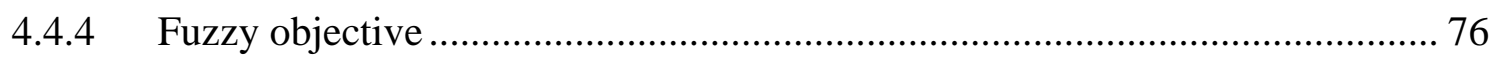

4.5 Algorithm of Successive Fuzzy MILP SCUC..................................................... 77

4.6 Test Results and Discussions ……………………............................................. 79

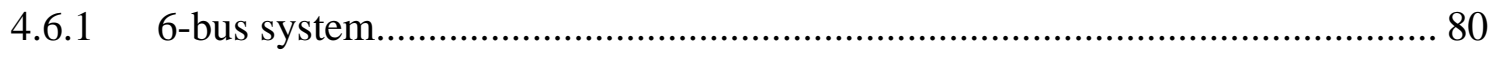

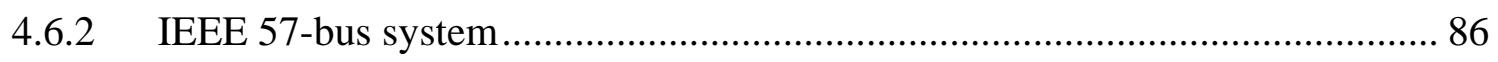

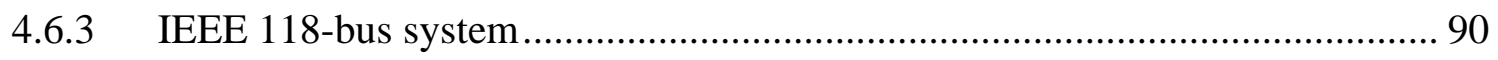

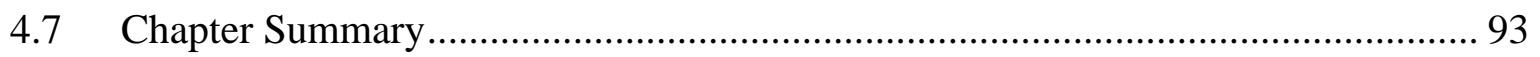


CHAPTER 5 FAst SECURITY AND Risk CONSTRAined PROBABILISTIC UNIT COMMITMENT

MEthod USING THE TAD MODEL OF WIND GENERATORS .................................................. 95

5.1 Security and Risk Constrained Probabilistic UC Algorithm............................... 96

5.1.1 Deterministic MILP Formulation of SCUC with WEGs ................................ 96

5.1.2 Formulation of SRCPUC with TAD model............................................ 100

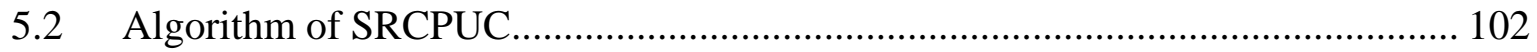

5.3 Test Results and discussions ................................................................. 104

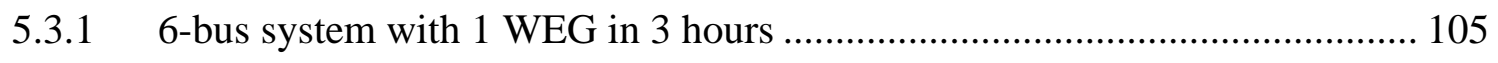

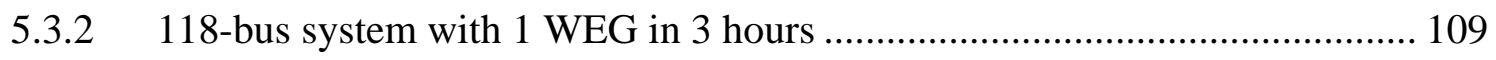

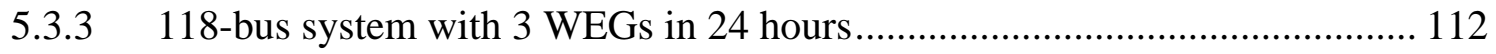

$5.4 \quad$ Chapter Summary ................................................................................. 116

CHAPTER 6 CONCLUSION AND FUTURE WORK............................................................. 118

6.1 Conclusion and Major Contributions .......................................................... 118

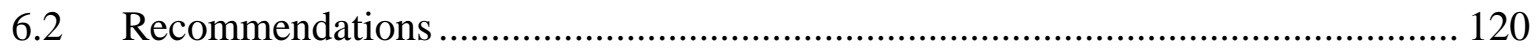

APPENDIX A FUNDAMENTALS AND TECHNIQUES....................................................... 122

A.1 Classical Enumeration Method ............................................................... 122

A.2 Power Balance Equations ...................................................................... 126

A.2.1 AC Power Balance Equations................................................................ 126

A.2.2 DC Power Balance Equations............................................................... 128

A.3 Formulations of Transmission Security Constraints ...................................... 129 
A.3.1 AC Transmission Security Constraints .................................................... 129

A.3.2 DC Transmission Security Constraints ..................................................... 131

A.4 Successive Linear Programming .................................................................. 132

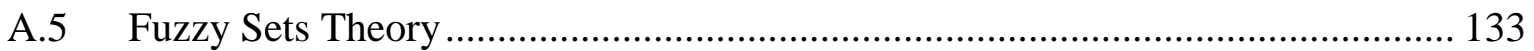

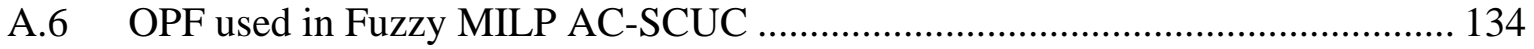

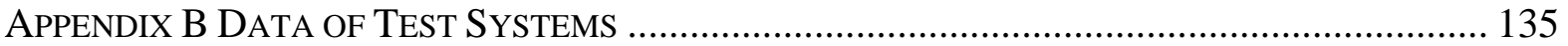

B.1 Six-Bus System with One WEG …............................................................. 135

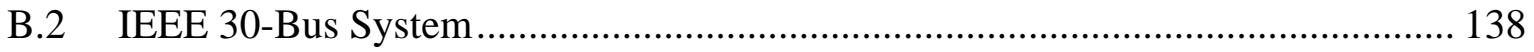

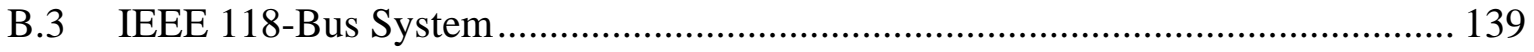

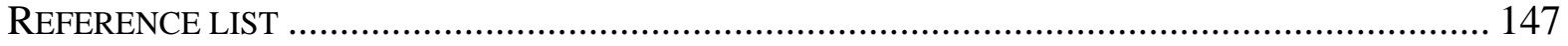




\section{LIST OF TABLES}

Table 3.1 Total Costs and Execution Times of Two Methods for the 30-Bus System........... 48

Table 3.2 Total Costs and Execution Times of Two Methods for the 118-Bus System......... 53

Table 3.3 Total cost with different $\sigma$ for 30 and 118-bus systems .................................. 57

Table 4.1 Details of 6-bus System with transmission outage in 3 hours ............................ 84

Table 4.2 Generation Schedules of 6-bus system in the final LPMOVE of 3 cases.............. 85

Table 4.3 Details of 57-bus System with transmission outage in 24 hours ......................... 88

Table 4.4 Transmission line MVA flows of 57-bus system for two states........................... 89

Table 4.5 Details of 118-bus System with transmission outage in 24 hours ....................... 93

Table 5.1 Generation scheduling and total cost in the proposed SRCPUC Algorithm ........ 106

Table 5.2 Results of proposed SRCPUC method with TAD model of WEGs compared to the

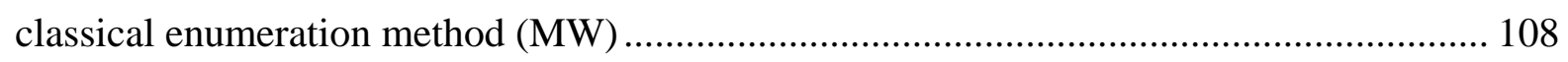

Table 5.3 Costs, EENS, reserve, and execution times of the 6-bus system........................ 108

Table 5.4 Costs and execution times of the 118-bus system with one WEG ..................... 111

Table 5.5 Result of 118-bus System with 3 WEGs in 24 hours ...................................... 114

Table B.1 CEG data of the 6-bus system .................................................................. 136

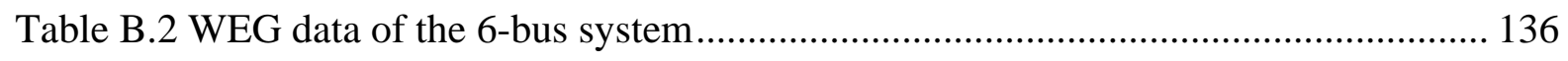

Table B.3 Load data of 6-bus system.................................................................... 137

Table B.4 Network data of the 6 -bus system ............................................................ 137

Table B.5 CEG data of IEEE 30-Bus System........................................................... 138 
Table B.6 WEG Data of IEEE 30-Bus System........................................................... 139

Table B.7 WEG Data of IEEE 118-Bus System in 3 hours........................................... 139

Table B.8 Forecasted output of WEGs in the 118-bus system (MW) in 24 hours .............. 140

Table B.9 CEG data of IEEE 118-Bus System......................................................... 141 


\section{LIST OF FIGURES}

Fig. 1.1 World total wind energy installed capacity in 11 years........................................ 2

Fig. 1.2 Wind power installed capacity (MW) of leading nations in 2011........................... 2

Fig. 1.3 Probability density function curve of wind power ........................................... 6

Fig. 1.4 Risk quantity using normal probabilistic distribution of wind power ...................... 7

Fig. 1.5 Generation scheduling process used by one ISO ............................................. 10

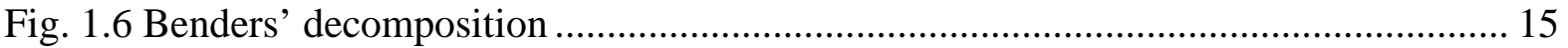

Fig. 2.1 TAD Model and the PDF curve of normal distribution........................................ 23

Fig. 2.2 Probability calculation of the TAD model ......................................................... 24

Fig. 2.3 Probability of wind power computed by normal distribution and the TAD model... 27

Fig. 2.4 EENS of wind power around the mean value (50MW) ....................................... 29

Fig. 2.5 Total cost changes with different $\sigma$ for the 2 -unit system.................................... 32

Fig. 3.1 Flowchart of proposed OPF method with TAD model ......................................... 44

Fig. 3.2 Costs of 1000 scenarios of 30 -bus system..................................................... 47

Fig. 3.3 Comparison of real power LMPs at all buses of the 30 -bus system........................ 49

Fig. 3.4 Total cost and output of WEGs with different $\alpha$ for the 30 -bus system ................. 50

Fig. 3.5 Reserve cost and EENS with different $\alpha$ for the 30-bus system ........................... 50

Fig. 3.6 Costs of 1000 scenarios of 118 -bus system ...................................................... 52

Fig. 3.7 Comparison of real power LMPs at all buses of 118-bus system .......................... 54

Fig. 3.8 Total cost and WEGs’ output with different $\alpha$ values of the 118-bus system .......... 55 
Fig. 3.9 Reserve cost and EENS with different $\alpha$ values of the 118-bus system 55

Fig. 4.1 Fuzzy set of Total Cost increment ................................................................. 73

Fig. 4.2 Fuzzy set for Line Flow Limit Constraint .................................................... 74

Fig. 4.3 Fuzzy set for Load Bus Voltage Upper Limit Constraint...................................... 75

Fig. 4.4 Fuzzy set for Load Bus Voltage Lower Limit Constraint .................................... 76

Fig. 4.5 Flowchart of the proposed AC-SCUC algorithm ............................................ 78

Fig. 4.6 Convergence characteristic of the 6-bus with no transmission outage .................... 81

Fig. 4.7 Convergence characteristic of the 6-bus with one connection out ......................... 82

Fig. 4.8 Convergence characteristic of the 6-bus with two connections out ........................ 84

Fig. 4.9 Convergence characteristic of the 57-bus without outage ................................... 87

Fig. 4.10 Convergence characteristic of the 57-bus with one line out................................ 87

Fig. 4.11 Convergence characteristic of the IEEE 118-bus without outage ......................... 91

Fig. 4.12 Convergence characteristic of the IEEE 118-bus with one line out ...................... 92

Fig. 5.1 Flowchart of the proposed SRCPUC algorithm with TAD model ........................ 103

Fig. 5.2 Total cost and cost of CEGs of 118-bus with 1-WEG in 3 hours ......................... 110

Fig. 5.3 Reserve cost and cost of the WEG of 118-bus with 1-WEG in 3 hours................. 110

Fig. 5.4 Total cost and cost of CEGs of 118-bus with 3 WEGs in 24 hours ...................... 113

Fig. 5.5 Reserve cost and cost of the 3 WEGs of 118 -bus in 24 hours.............................. 113

Fig. 5.6 Total cost and wind energy with various standard deviation (118-bus, 3-WEG, 24-

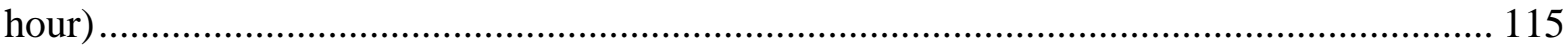

Fig. A.1 Probability density and cumulative distribution functions in classical enumeration

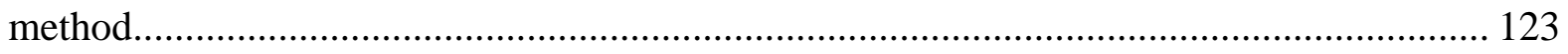

Fig. A.2 Scenarios production of power outputs of WEGs for OPF/SCUC process............ 124 
Fig. A.3 Flowchart of the classical enumeration ......................................................... 125

Fig. A.4 Net power injected into the transmission system by the $i^{\text {th }}$ bus.......................... 127

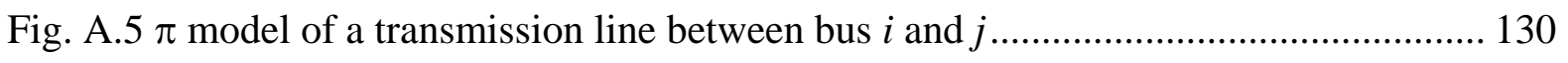

Fig. B.1 Six-bus Power System with one WEG …................................................... 135 


\section{LIST OF SYMBOLS}

\section{Definition of Convention:}

$\overline{\mathrm{Var}}$, Var Upper and lower bars indicate upper and lower limits of the generic variable ' $V a r '$

$\operatorname{Var}^{0} \quad$ ' 0 ' indicates initial or current state of the generic variable 'Var'

$\Delta \operatorname{Var} \quad$ ' $\Delta$ 'indicates incremental change in the generic variable 'Var'

$\mu \operatorname{Var}(U / L) \quad$ Fuzzy membership variable respect to variable 'Var' (refer to upper/lower limit)

Variables of wind electric generators:

$\alpha \quad$ Fraction of expected energy not served (EENS) to quantify uncertainty of wind electric generators (WEG)

cp Vector (matrix) of probability of output of WEGs calculated by normal probabilistic distribution

$c p^{T} \quad$ Vector (matrix) of probability of output of WEGs calculated by triangular approximate distribution model

E $\quad$ Vector (matrix) of EENS

pd Vector (matrix) of probability density of output of WEGs

$\sigma \quad$ Vector (matrix) of standard deviation of forecasted of output of WEGs

$\overline{\overline{P G}} \quad$ Vector (matrix) of mean values of forecasted output of WEGs 
WG $\quad$ Number of WEGs

\section{Variables of OPF and day-ahead UC:}

$a c, b c \quad$ Vectors of fixed unit costs $(\$)$, energy incremental costs (\$/MWh) of conventional electric generators (CEG)

$a w, b w \quad$ Vectors of fixed unit costs (\$), energy incremental costs (\$/MWh) of WEGs

$\beta \quad$ Fraction of total hourly demand to quantify generation failure of CEGs

$C G, M \quad$ Numbers of CEGs and cost segments in the cost versus power output of a CEG

$\gamma \quad$ Fraction of total hourly reserve to be scheduled by spinning reserve

G10, P10 Index and capacities of quick start (10 min) units

IC Vector of initial conditions of units

MR Vector of hourly required reserve capacity for the system

$N B, \quad N T \quad$ Numbers of buses and transmission lines

NH, Number of hours

$P G, P D \quad$ Vectors (matrices) of real power generation and demand

$P G^{\text {Sch }} \quad$ Vectors (matrices) of scheduled real power generation

$P G^{\text {Act }} \quad$ Vectors (matrices) of actual real power generation

PM Matrix of real power output of cost segments

$R 10, R 60 \quad$ Vectors of 10 -minute and 60 -minute ramp rates

rc, sc Vectors of reserve costs (\$/MWh) and start-up costs (\$/start)

SR Vector (matrix) of online spinning reserves

$t, n, m, i \quad$ Indices for hour, generator, segment of a generator, and bus numbers

$U, S U \quad$ Matrix of binary integers representing unit and start-up status 
UT, DT Vectors of minimum up time and minimum down time of units

\section{Variables of AC/DC transmission security constraints:}

BI Inverse of bus susceptance matrix

GI Set of indices of generators connected on buses

$j, k \quad$ Indices for bus numbers

J1, J2, J3, J4 3-dimensional sub-matrices of Jacobian, each layer corresponds to an hour $J D L, J V L \quad$ Sensitivity matrices relating incremental bus phase angles and voltage magnitudes to changes in MVA line flows

$l \quad$ Index for transmission lines

$\lambda \quad$ Fuzzy objective function

PL Vectors (matrices) of real power line flows of DC transmission constraints

$P T, Q T \quad$ Vectors (matrices) of net real and reactive powers flowing from buses into connected transmission system

$\Theta \quad$ Matrix relating the net real power injection to real power line flow

$Q G, Q D \quad$ Vectors (matrices) of reactive power generation and demand

SF $\quad$ Vector (matrix) of MVA line flows

$S L, S B \quad$ Vectors (matrices) of MVA branch line flows in $\pi$ model

$V, \delta \quad$ Vectors (matrices) of bus voltage magnitudes and phase angles

$V G, V L \quad$ Vectors (matrices) of bus voltage magnitudes of generators and loads: $\mathrm{V}=$ [VG VL]

$Y, B^{\prime} \quad$ Matrices of bus admittance and bus susceptance

$y_{l}, y_{s} \quad$ Vectors of transmission line admittance and shunt admittance in $\pi$ model 


\section{ChAPTER 1}

\section{INTRODUCTION}

Modern power systems are changing to integrate increasing amounts of renewable energy sources. Many jurisdictions, such as the European Union, have aggressive targets to generate $20 \%$ of their supplies from renewable energy sources by the year of 2020 [1]. Renewable energy sources with the most mature technologies are that of wind and solar energy. Hence, there is a huge focus on ensuring that the current power system analysis and optimization algorithms accommodate these technologies [2].

Wind energy is widely utilized in the world because it is one of the most safe, environmentally friendly, and renewable energy resources. By the end of the year 2011, international wind power capacity had reached 239 GW [3], as shown in Figure 1.1. Wind generator installations are growing rapidly, especially in China, USA, Germany, Spain, and India, which cumulatively represent $74 \%$ of the global installed wind capacity, as shown in Figure 1.2. Although these nations enjoy environmental and economic benefits from green energy, independent system operators (ISO) encounter challenges while managing daily power system operations with significant amounts of renewable energy due to their uncertainty, variability and such other characteristics. 


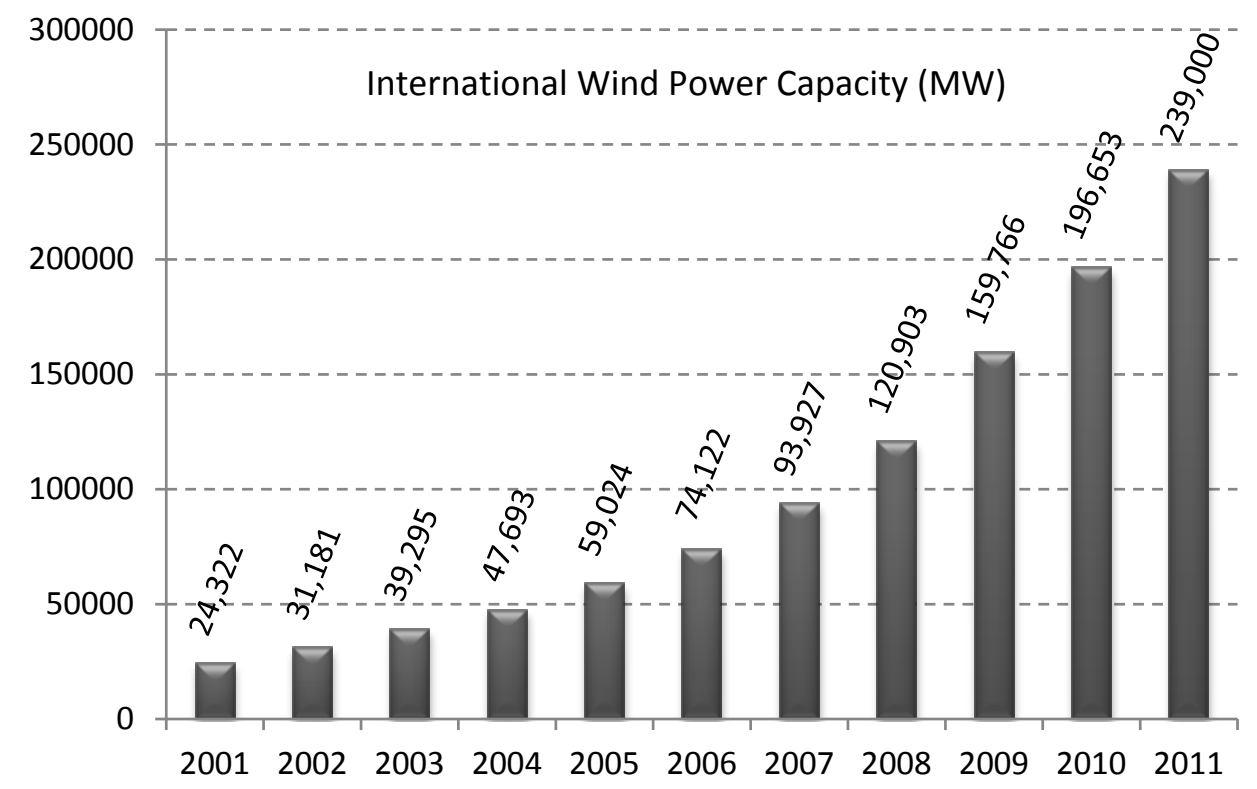

Fig. 1.1 World total wind energy installed capacity in 11 years

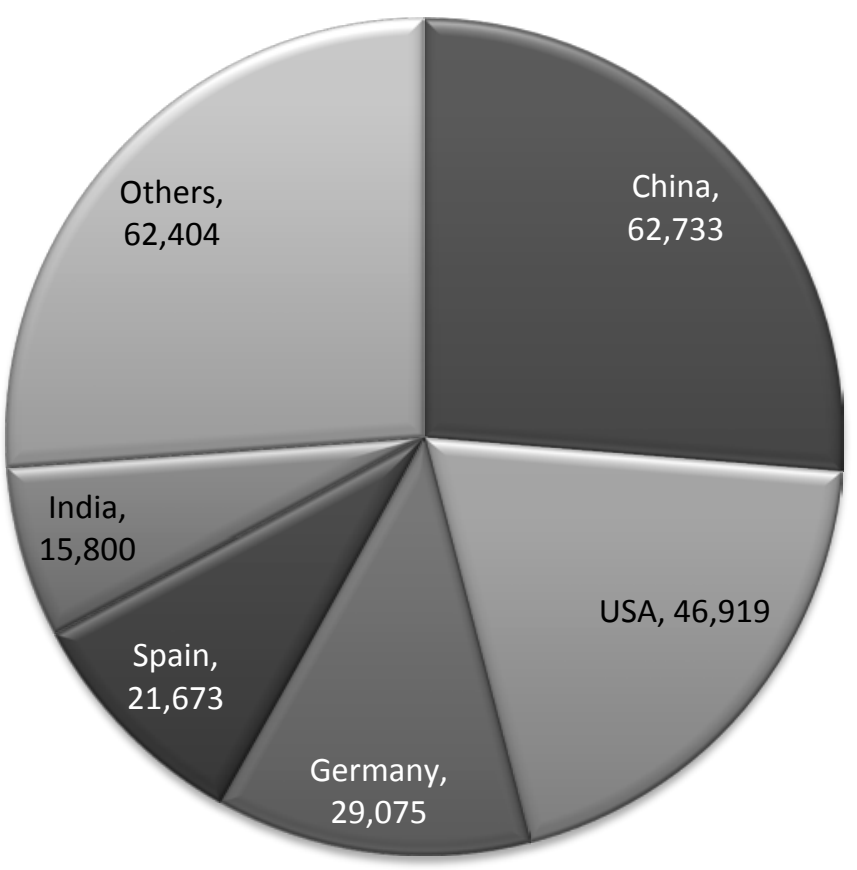

Fig. 1.2 Wind power installed capacity (MW) of leading nations in 2011 
As more wind electric generators (WEG) are being installed and used in power systems with complex transmission systems, unpredictable nature of wind energy increases the risk of possible power shortages that will consequently result in failure to supply contracted loads. The uncertainties in power output of WEGs create additional risks in the near-term optimal generation schedule when incorporating WEGs into electric power systems. ISOs require flexible and accurate scheduling techniques when using WEGs alongside all other kinds of generators in the short-term time frame (from a few hours up to 24 hours) to contend with the risk introduced by the uncertainties involved in this renewable energy source.

In addition, most power systems are faced with adapting their energy supply mix to accommodate an increasing amount of wind energy. Transmission systems, which were planned in the past to operate with conventional power generation, must now connect and operate with renewable energy sources as well on a significant scale. This change causes transmission systems to operate in new states that were not seen before. Thus, it is very important that ISOs take into account AC transmission network constraints in their daily power system scheduling algorithms.

Many techniques and methodologies are used to model wind power output in power system optimization algorithms. For example, the risk of failing to supply the contracted load can be quantified as expected energy not served (EENS). In this dissertation, the probabilistic aspect of wind power will be explored, modeled, and incorporated in power system optimization algorithms such as optimal power flow (OPF) and day-ahead unit commitment (UC). The probabilistic models of power output of WEGs and the associated EENS formulae will be discussed in the next section. 


\subsection{Probabilistic Modeling of Power Output of Wind Electric Generators}

Since the late 1980s, the process of incorporating WEGs into optimization algorithms to manage the daily operation of power systems has been developed extensively in academic research [4]. Reference [5] reviews many wind power forecasting techniques, which can generally be divided into two main categories: 1) physical prediction methods or 2) traditional statistic methods (or called learning approaches).

While physical prediction models are designed using weather and geographic data at the location of the WEGs, traditional statistic methods are used to probabilistically represent wind power output by linking historical data of power output with weather parameters to forecast future power output, performing a one-step conversion usually described as a 'black box' [5]. These probabilistic models of WEG power output based on traditional statistical methods have been more widely used in power system optimization algorithms than the physical prediction models.

Probabilistic models of wind energy such as the Weibull distribution [6]-[9] and normal probabilistic distribution [10]-[12] are commonly used to represent forecasts of wind power. The Weibull distribution is used for long-term probabilistic wind speed representation. The normal probabilistic distribution is used for short-term, one hour to a day ahead, to represent wind power forecasting error. Furthermore, probabilistic models are used to quantify possible EENS values that arise from probable shortfall of power output of WEGs from forecasts. Such models are very important to understand the influence of the variability of wind power on all aspects of optimally operating power systems. 
This dissertation focuses on short-term power system optimization algorithms. This research has used a probabilistic model of wind power based on normal probabilistic distribution to represent the power output of WEGs in power system optimization algorithms, as it is the best method to effectively describe the energy forecast in this time horizon. The details of probabilistic modelling of power output of WEGs and the formula used to compute the EENS value are presented below.

\subsubsection{Normal Probabilistic Distribution}

The normal probabilistic distribution is characterized by two parameters: mean value and standard deviation $(\sigma)$ [13]. The mean value represents the expected value of wind power output in this forecast from a WEG. The standard deviation value indicates the possible variation from the expected power output. Figure 1.3 shows a typical probability density function (PDF) curve for a normal probabilistic distribution with possible wind power output from a WEG.

The PDF is a nonlinear exponential function of wind power (1.1).

$p d=\frac{1}{\sigma \sqrt{2 \pi}} e^{\frac{-(P G-\overline{\overline{P G}})^{2}}{2 \sigma^{2}}}$

This formula represents the probability density of wind power at a value of $P G$. The area under the curve of the PDF is equal to 1, denoting all possible wind power values that can be supplied by the WEG in that hour. The risk of failure for a WEG to supply a certain value of power output can be computed using the PDF of the normal probabilistic distribution associated with wind power output forecast, and the risk can be quantified as an EENS value. 


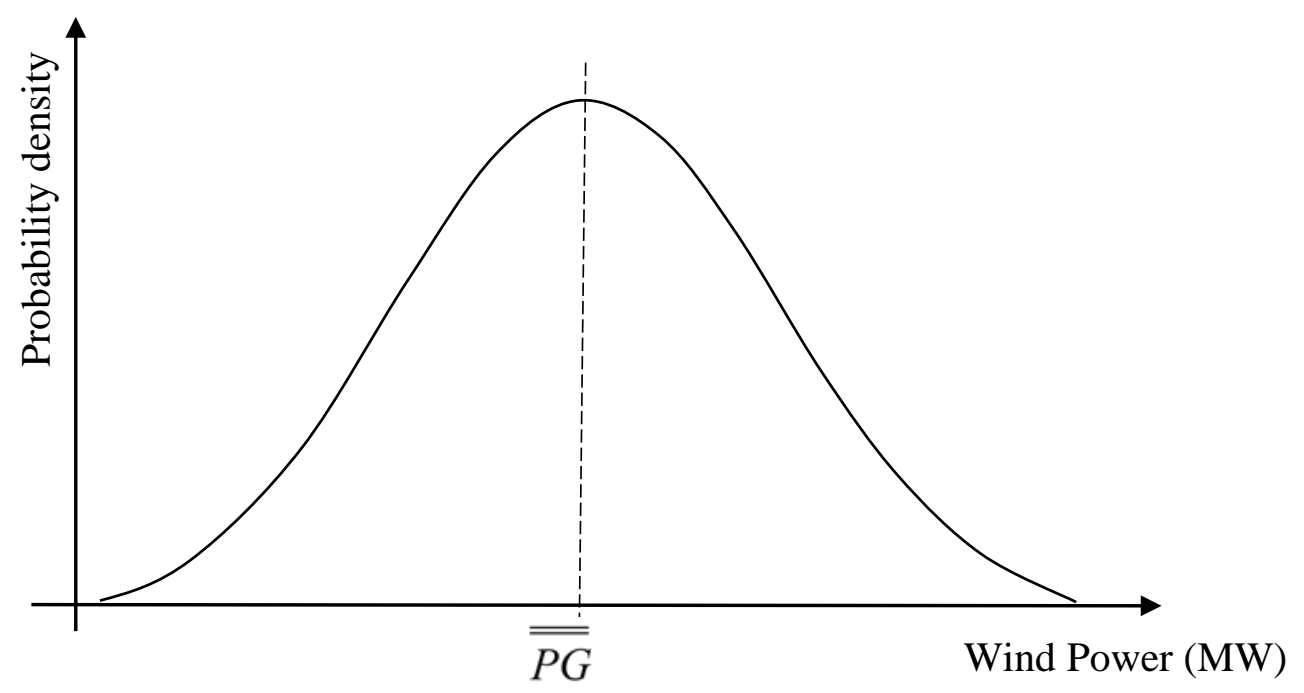

Fig. 1.3 Probability density function curve of wind power

\subsubsection{Expected Energy Not Served}

If the PDF of power output from a WEG is known, then an EENS value can be computed for a given power output. During the generation scheduling procedure, consider that an amount of power $(P G)$ is scheduled from the WEG for a particular hour, shown in Figure 1.4.

Under the PDF curve, the shaded area to the right of $P G$ represents the probability that wind power output will be greater than or equal to the amount of $P G$. The hatched area to the left of PG quantifies the probability that the WEG's output would be less than $P G$. This hatched area is a function of $P G$ and it is the upper bound of the probability for the power output to be less than $P G$. It may be computed as below: 
$c p=\int_{0}^{P G} \frac{1}{\sigma \sqrt{2 \pi}} e^{-\frac{(x-\overline{\overline{P G}})^{2}}{2 \sigma^{2}}} d x$

In the hatched area, the WEG cannot supply the amount of power $(P G)$. The EENS value that quantifies risk of failure to supply a power output of $P G$ can be computed by multiplying the hatched area in Figure 1.4 with its corresponding PG [6]. After computing the EENS value, sufficient reserves could be scheduled for the system to overcome such a risk.

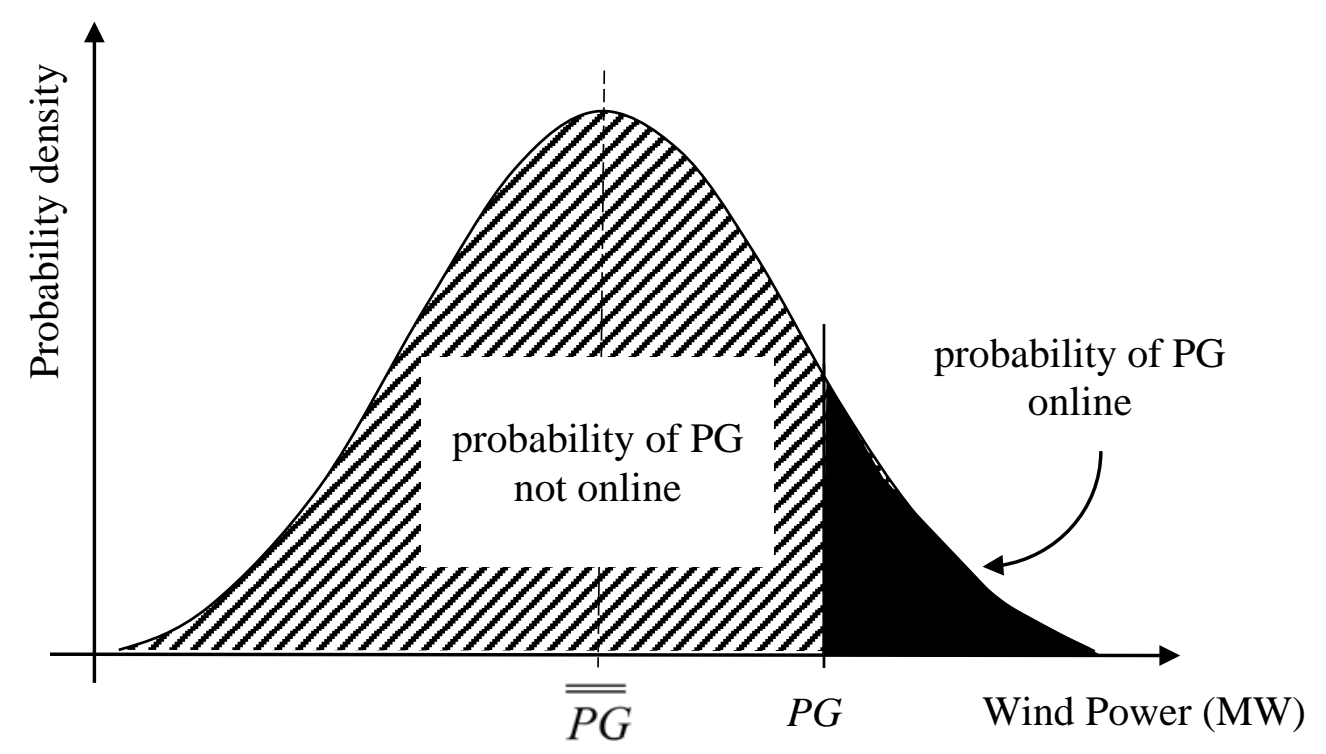

Fig. 1.4 Risk quantity using normal probabilistic distribution of wind power

However, the computation of EENS requires integrating the PDF of normal probabilistic distribution, which is a nonlinear exponential function. Incorporating such a complex function 
into large-scale optimization formulations of OPF or day-ahead UC is very challenging. Furthermore, this challenge is compounded when the number of WEGs is large.

\subsection{Challenges in probabilistic modeling of wind electric generators}

As presented in last section of this chapter, the PDF of power output from WEGs and the EENS function are formulated as an exponential function and an integration function of wind power respectively. Due to the nonlinear and stochastic characteristics of the probabilistic models of power output of WEGs based on normal probabilistic distribution function, it is difficult to incorporate them into OPF and day-ahead UC algorithms.

Sampling methods, the Monte Carlo simulation [8][11], and the Latin Hypercube simulation techniques [10][12] can be utilized to produce wind power output scenarios. Using mean values of forecasts and standard deviations, a large set of random numbers is generated to represent possible power output values. The probability of each possible power output can be computed by using these random outputs. The optimum system output is determined and the system security is analyzed using this large set of possible WEG power outputs. However, these methods are besieged with a huge volume of data sets and are computationally challenged. Further, this discrete approach cannot fully characterize the stochastic distribution pattern of wind power output.

In order to probabilistically represent output of WEGs in OPF and UC formulations, researchers can resort to the classical enumeration technique [14], which is not a stochastic sampling method. Consider a power system with ' $n$ ' WEGs. Power output of each WEG can be represented by a normal PDF that is divided into ' $m$ ' segments. The number of executions 
in a classical enumeration technique required to compute the probabilistic output is equal to $m^{n}$ (as detailed in Appendix A). Through this expression, it can be clearly seen that as the number of WEGs increases, the number of executions will increase exponentially and become computationally cumbersome.

The use of this classical enumeration technique for computing probabilistic output is difficult for offline applications, while probabilistic OPF and probabilistic UC algorithms are computationally even more demanding as they are meant for real-time and near real-time applications that incorporate stochastic information of power output of WEGs.

Hence, in view of these shortcomings of the existing methods reported in the literature, an accurate probabilistic model of power output of WEG needs to be developed to effectively minimize the EENS value of WEGs and its associated costs in daily power system optimization algorithms, such as probabilistic OPF and probabilistic UC.

\subsection{Review of Probabilistic Optimal Power Flow}

The OPF algorithm [15] is an important optimization tool for short-term generation scheduling and determination of locational marginal prices (LMP). This topic has been extensively researched over the last 50 years, with researchers developing formulations to determine optimal solutions by minimizing total operation costs and/or total system real power losses. The OPF algorithm computes the optimal real power generation schedule to ensure that generation costs are kept to the minimum while maintaining bus-wise power balance and enforcing transmission network limits and other operational constraints [15]-[25].

Figure 1.5 shows a typical generation scheduling process utilized by one of the ISOs in North 
America [6].

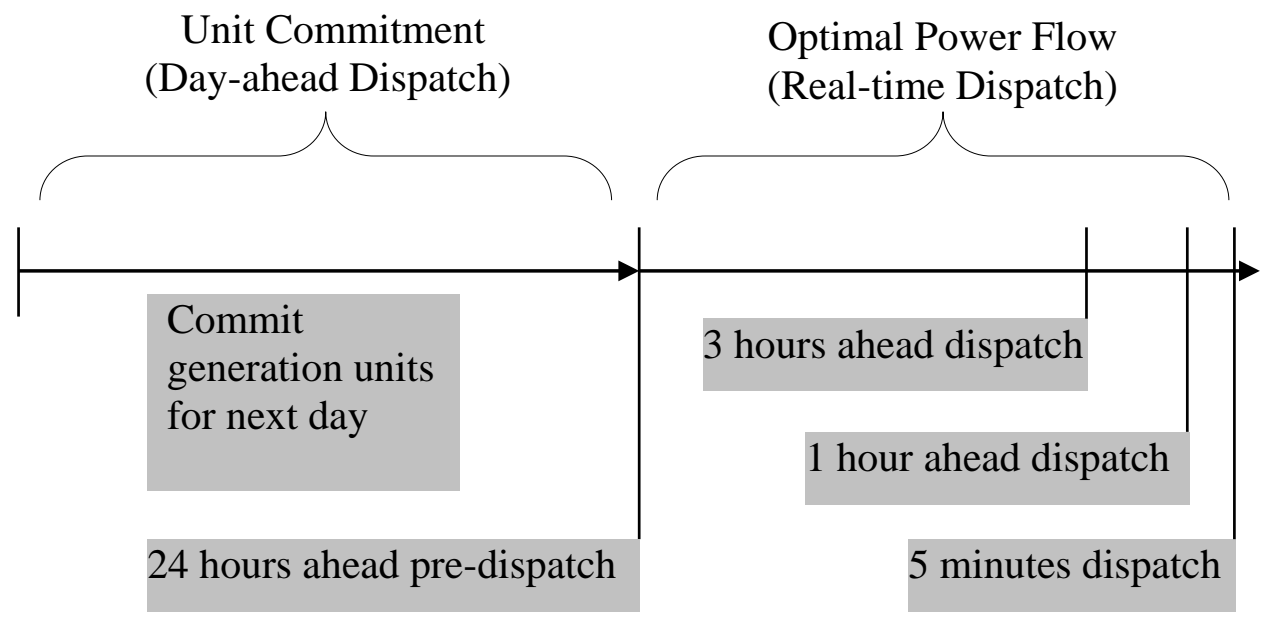

Fig. 1.5 Generation scheduling process used by one ISO

The OPF algorithm is mainly used for near-real-time and real-time dispatch (5 minutes to a few hours ahead), shown as the right part of the generation dispatch procedure in Figure 1.5. Data of system demands and intermittent generations are updated between each OPF solutions to increase accuracy. Available generation units are dispatched using the OPF algorithm to supply the actual demand in order to satisfy immediate system needs.

Traditionally, the OPF algorithm has been developed using nonlinear programming (NLP) technique [26]-[29] and successive linear programming (SLP) technique [30], [31]. However, methods based on SLP technique have become more widely adopted in commercial grade implementation due to their robustness in finding optimal solutions. 
The probabilistic OPF is an important extension of the OPF algorithm that accounts for uncertainties in power systems [32]. Two different research directions have emerged in the area of probabilistic OPF while modeling uncertainty [33]:

1) The first approach solves OPF formulations using probabilistic analysis methods such as the First-Order Second-Moment method [34], the Cumulant method [35], and the Two-Point Estimate method [36], etc. These methods consider median values of uncertain variables to solve a chosen deterministic OPF formulation. The optimal state determined for the median case along with other probabilistic information such as variance, cumulants, etc. are then used to estimate uncertainty in the optimal solution.

2) The other approach is to directly include probabilistic information in the objective function and constraints and solving them using conventional methods [37] [38].

Currently, both research directions model the uncertainties of inputs through random-based techniques, such as the Monte Carlo simulation [32], but these techniques are either time consuming or lose accuracy when applied in practical situations. Hence, there is an urgent need in the field for a practical fast probabilistic OPF method.

As an important consideration in parallel to OPF, the UC algorithm must also incorporate the probabilistic model of WEGs to improve accuracy and efficiency in daily operation. A review of the probabilistic UC algorithm is presented in the following section. 


\subsection{Review of Probabilistic Unit Commitment}

Day-ahead UC is performed to generate pre-dispatch schedule for the next block of time, such as 24 hours. In the UC process, generation units are committed to ensure that the system has an adequate amount of energy sources for the next 24 hours and that the optimal generation schedule is feasible with respect to inter-temporal constraints such as minimum up times, minimum down times and ramp rate limits. These inter-temporal constraints bind all the 24 hourly economic dispatch solutions. On the dispatch day, the committed generation units are re-dispatched every hour using a real-time OPF algorithm to meet the real-time demand.

The UC process is usually formulated as a quadratic or a mixed integer programming (MIP) problem with linear and nonlinear constraints. A large number of techniques and methods have been applied to solve this problem [39]-[42]. As progress has been made on computational methods, optimization solvers, and hardware availability, the industry standard has progressed towards the use of MIP to obtain a robust and efficient solution method [43].

In 1999, Pennsylvania, Jersey and Maryland (PJM) — an ISO operating in the USA — field-proved the advantage of using MIP to solve its day-ahead UC problem compared with older optimization engines such as Lagrangian relaxation method, demonstrating the significant economic benefit of using MIP. Since 2004, PJM has implemented this MIP-based method to schedule its day-ahead market. Subsequently, most other major ISOs in USA such as California ISO, Midwest ISO, and ISO-New England use this method. Others such as New York ISO and Southwest Power Pool are also planning to update their day-ahead UC algorithm to use a MIP-based optimization engine. 
An important variant of the MIP model is the mixed integer linear programming (MILP) model that only addresses linear objectives and constraints. It has recently drawn the attention of researchers [44] and is currently being utilized by some ISOs in their optimization software [43]. In order to align the efforts of this research with the current state-of-the-art industrial practice and to effectively manage the demanding requirements of modern power systems, the MILP formulation is used in this dissertation to model day-ahead UC problems.

In power systems with high wind energy penetration, ISOs require accurate and computationally efficient techniques and tools, which probabilistically model power output of WEGs to solve the day-ahead UC problem. Similar to probabilistic OPF algorithms, a probabilistic UC formulation must account for wind power and its uncertainties.

The problem of incorporating WEGs into probabilistic UC has been investigated by many researchers. Typically, Monte Carlo simulation and Latin Hypercube simulation methods are used to produce wind power output scenarios for probabilistic UC [8], [10]-[12]. However, these methods consider WEGs as non-dispatchable units and multiple deterministic problems are solved for different scenarios of wind power output. These methods are computationally burdensome and are not practical for real-time or near real-time power system operations.

In order to maximize profit, WEGs must meet their forecasted power outputs. As an operational safeguard, reserves are simultaneously scheduled to counter the risks caused by uncertainties in wind power. This approach requires that a practical probabilistic UC algorithm has the capacity to minimize both costs and risks, but such a requirement cannot be explicitly performed by scenario-based methods. Hence, a probabilistic UC algorithm is needed that 
incorporates advanced probabilistic wind power models to minimize the total generation cost and minimize the total risk of failure to provide pre-arranged power loads.

In addition, in order to provide a valid starting state to a near real-time OPF algorithm, a dayahead UC algorithm must have the capability to produce a solution that is transmission network feasible. Therefore, a UC algorithm must produce an optimal solution that is feasible with respect to transmission network constraints. Accordingly, security constrained unit commitment (SCUC) methods that incorporate AC transmission security constraints are reviewed in the next section.

\subsection{Review of Security Constrained Unit Commitment}

The complex numerical challenge of the SCUC problem with AC transmission security constraints (AC-SCUC) has been studied extensively over the past four decades [45]. In recent years, advances in computational hardware and software tools have enabled more powerful algorithmic development in solving this challenge. Researchers have published a few works to solve the AC-SCUC problem using techniques such as Benders' decomposition, which decompose the problem into master and slave sub-problems [46]-[53].

The AC-SCUC algorithm using Benders' decomposition technique is shown in Figure 1.6. The master problem is usually decision-making, which produces unconstrained generation schedules. The security constraints are solved in sub-problems using a power flow algorithm or an OPF algorithm. Benders' cuts are created from violated constraints to be incorporated into the master problem if one or more sub-problems are not feasible. The master problem is 
resolved with these additional constraints. This process continues until the optimal solution of the master problem satisfies all constraints.

In Benders' decomposition methods, the master and slave problems are solved back and forth to obtain a feasible solution. The UC problem and security constraints are independent of each other.

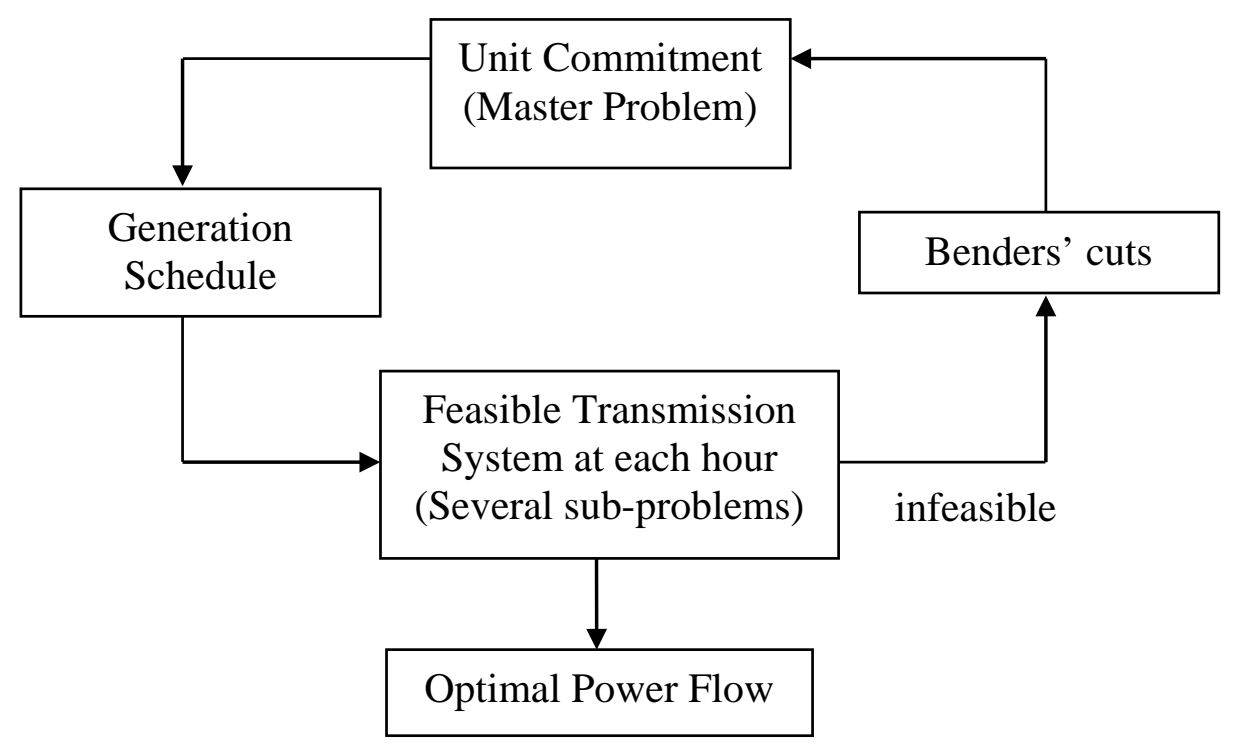

Fig. 1.6 Benders’ decomposition

A few other works have incorporated power flow equations and transmission security constraints directly into the SCUC formulation. In these cases, the authors used DC power flow equations to constrain UC in a mixed integer linear programming (MILP) formulation [6] [54] and solved the SCUC problem using MILP engines. However, the DC power flow equations 
are an approximation of AC power flow equations, as they are dependent upon a large set of assumptions, as detailed in Appendix A. Other authors transformed the MIP-based UC problem into a nonlinear convex problem by relaxing integer variables [55]; however, this method encounters difficulties due to the infeasibility caused by status variables being converted back to integers in the final stage of finding a practical solution. Thus, some other method must be used after solving the relaxed SCUC problem, or researchers risk more computational burden and loss of system optimality.

Furthermore, transmission contingency and outage planning problems in SCUC were studied by researchers using Benders’ decomposition technique [56]-[58]. Due to the limitation of such techniques, these authors considered scenarios in which feasible transmission solutions could be obtained, but did not achieve results capable of effectively scheduling generation in weak transmission systems, where there is currently no feasible solution to alleviate all transmission constraint violations.

Hence, a robust and flexible AC-SCUC formulation that directly includes AC transmission system is needed for day-ahead UC algorithm, to find the optimal solution with the least operating cost and that is transmission system feasible. This method must also have the capacity to handle infeasibility caused by severe conditions resulting from transmission outages.

\subsection{Objectives of the Study}

In order to stochastically model power output of WEGs and integrate those models into power systems optimization algorithms such as OPF and UC, and to solve practical 
optimization problems with AC transmission system constraints while minimizing costs and risks, the following objectives have been chosen for this dissertation:

1) Create a new triangular approximate distribution (TAD) model to stochastically represent wind power output and use this TAD model of WEGs to develop a simple method to compute forecast errors to quantify expected energy not served (EENS) by WEGs.

2) Develop a fast probabilistic OPF algorithm using the TAD model of WEGs and solve using the successive linear programming (SLP) technique.

3) Model the AC transmission system and related constraints in a mixed integer linear programming (MILP) security constrained unit commitment (SCUC) formulation. Solve this formulation using the SLP technique and use elements of fuzzy set optimization technique to improve computational efficiency and hasten convergence.

4) Integrate the TAD model of WEGs into an MILP formulation of SCUC algorithm to create a fast security and risk constrained probabilistic unit commitment method.

The outline of this dissertation is given as below.

\subsection{Chapter-wise Outline of the Dissertation}

This dissertation is organized in the following chapters:

In Chapter 2, the proposed TAD model of WEGs is presented and compared with normal probabilistic distribution function. An expression of EENS using the TAD model of WEGs is

derived and presented. Effects of standard deviation on EENS value are also studied and presented. 
In Chapter 3, a linear programming (LP) formulation of the fast probabilistic OPF algorithm with the TAD model of WEGs is developed and reported. The SLP technique used to solve this formulation is presented. Several sizes of power systems are tested with the proposed fast probabilistic OPF method with the TAD model of WEGs and reported.

In Chapter 4, a security-constrained MILP formulation of the UC problem with full AC transmission system representation is presented. Fuzzy set optimization and SLP techniques are used to solve the formulation. A complete solution method for this proposed formulation is presented in this chapter. Tests on various power systems and test results are presented and discussed.

In Chapter 5, a fast probabilistic unit commitment algorithm with transmission security constraints to minimize costs and risk is proposed. The TAD model of WEGs is integrated into a MILP formulation of the UC problem to create this probabilistic optimization formulation. An algorithm for solving this proposed probabilistic, security constrained UC formulation to minimize operation costs and risk from uncertainty is reported. Tests on various systems and their results are reported.

In Chapter 6, conclusions and possible future research directions are presented.

\subsection{Chapter Summary}

The major motivations and methodologies of this $\mathrm{PhD}$ research have been presented in this chapter. Certain key publications reported in literature for OPF and UC were reviewed in this chapter. Probabilistic aspect of wind energy, their importance in power systems optimization 
and basic probabilistic models were presented. The detailed procedures and accomplishments of this research will be presented and discussed in the chapters that follow. 


\section{CHAPTER 2}

\section{Triangular ApProximate Distribution MODEL}

\section{OF WEGS}

The triangular approximate distribution (TAD) model of wind electric generators is proposed in this chapter. The TAD model approximates the probability density function (PDF) of normal probabilistic distribution that is most often used to model short-term forecast of wind power. The time horizon is typically from one hour to 24 hours into the near future. Thereafter, this TAD model is used to develop an expression for a cumulative distribution function (CDF). The CDF expression developed using the TAD model quantifies the probability of uncertainty in the forecast and it is extended to develop an expression for the expected energy not served (EENS) resulting from the uncertainty of real power output of WEGs. The EENS function using normal probabilistic distribution is given in the first section of this chapter, and is then compared to the expression of EENS that is developed from the TAD model. 


\subsection{EENS of WEGs using Normal Distribution}

The PDF of wind power forecast is a normal distribution. It can be expressed as an

exponential function of the best forecasts $\left(\overline{\overline{P G_{n}}}\right)$ of the $n^{\text {th }}$ WEG and corresponding standard deviation value $\left(\sigma_{n}\right)$ as shown below:

$p d_{n}=\frac{1}{\sigma_{n} \sqrt{2 \pi}} e^{\frac{-\left(P G_{n}-\overline{\overline{P G_{n}}}\right)^{2}}{2 \sigma_{n}^{2}}} \forall n \in W G$

Integrating from zero to a particular power output of the WEG $\left(P G_{n}\right)$, the CDF of wind power may be defined as below.

$c p_{n}=\int_{0}^{P G_{n}} \frac{1}{\sigma_{n} \sqrt{2 \pi}} e^{\frac{-\left(x-\overline{\overline{-P G_{n}}}\right)^{2}}{2 \sigma_{n}^{2}}} d x \forall n \in W G$

The value of $c p_{n}$ in equation (2.2) represents the probability that the power output of the $n^{\text {th }}$ WEG is lower than the particular power output value $\left(P G_{n}\right)$. It may also be considered as the probability that the particular power output $\left(P G_{n}\right)$ cannot be supplied by this WEG.

In the real-time operation, actual power $\left(P G^{A c t}\right)$ supplied by the WEG may not same as the power scheduled an hour ahead $\left(P G^{S c h}\right)$. EENS can be estimated as the following (2.3):

$E_{n}=\int_{P G_{n}^{A c t}}^{P G_{n}^{S c h}}\left(P G_{n}^{S c h}-P G_{n}^{A c t}\right) \frac{1}{\sigma_{n} \sqrt{2 \pi}} e^{\frac{-\left(x-\overline{\overline{P G_{n}}}\right)^{2}}{2 \sigma_{n}^{2}}} d x \forall n \in W G$ 
However, as the view from the scheduling point, the actual power supplied in the real-time is not known and it is set to zero for simplicity. Therefore, the expression of EENS can be modified as (2.3.1).

$$
E_{n}=\int_{0}^{P G_{n}} P G_{n} \frac{1}{\sigma_{n} \sqrt{2 \pi}} e^{\frac{-\left(x-\overline{\overline{P G_{n}}}\right)^{2}}{2 \sigma_{n}^{2}}} d x
$$

As an approximation, that is an upper bound of above, the power generation, $P G_{n}$ is moved out of the integral to give:

$E_{n}=P G_{n} \cdot \int_{0}^{P G_{n}} \frac{1}{\sigma_{n} \sqrt{2 \pi}} e^{\frac{-\left(x-\overline{\overline{P G_{n}}}\right)^{2}}{2 \sigma_{n}^{2}}} d x=P G_{n} \cdot c p_{n} \forall n \in W G$

The modified EENS can be computed by multiplying power output $\left(P G_{n}\right)$ with the probability $\left(c p_{n}\right)$ that cannot supply the amount of power online.

It is observed that the modified EENS is a nonlinear integration function of wind power. Owing to its nonlinearity, such a function is not suitable for LP and MILP optimization formulations. Hence, the TAD model of WEGs is proposed to replace this complex nonlinear function so that it is amendable to linear optimization techniques. Details of the TAD model are given in the following sections.

\subsection{Triangular Approximate Distribution versus Normal Distribution}

The same best forecast value $\left(\overline{\overline{P G_{n}}}\right)$ and its corresponding standard deviation $\left(\sigma_{n}\right)$ are taken for the $n^{\text {th }}$ WEG. Considering the interval from $\left(\overline{\overline{P G_{n}}}-2.5 \sigma_{n}\right)$ to $\left(\overline{\overline{P G_{n}}}+2.5 \sigma_{n}\right)$ on the 
PDF curve of a normal probabilistic distribution, the area under the curve is nearly equal to 1.0 (0.988), which means that almost all the possible wind output values for a particular hour are captured within this interval of the PDF curve. This same interval in the TAD model also has an area close to $1.0(0.997)$. The value of 2.5 was determined by rounding $\sqrt{2 \pi}$. Due to this similarity, the TAD model is used to represent the probabilistic distribution of wind power forecast as shown in Figure 2.1. In addition, the probability of wind power not available can be computed and compared with normal PDF. This is presented below.

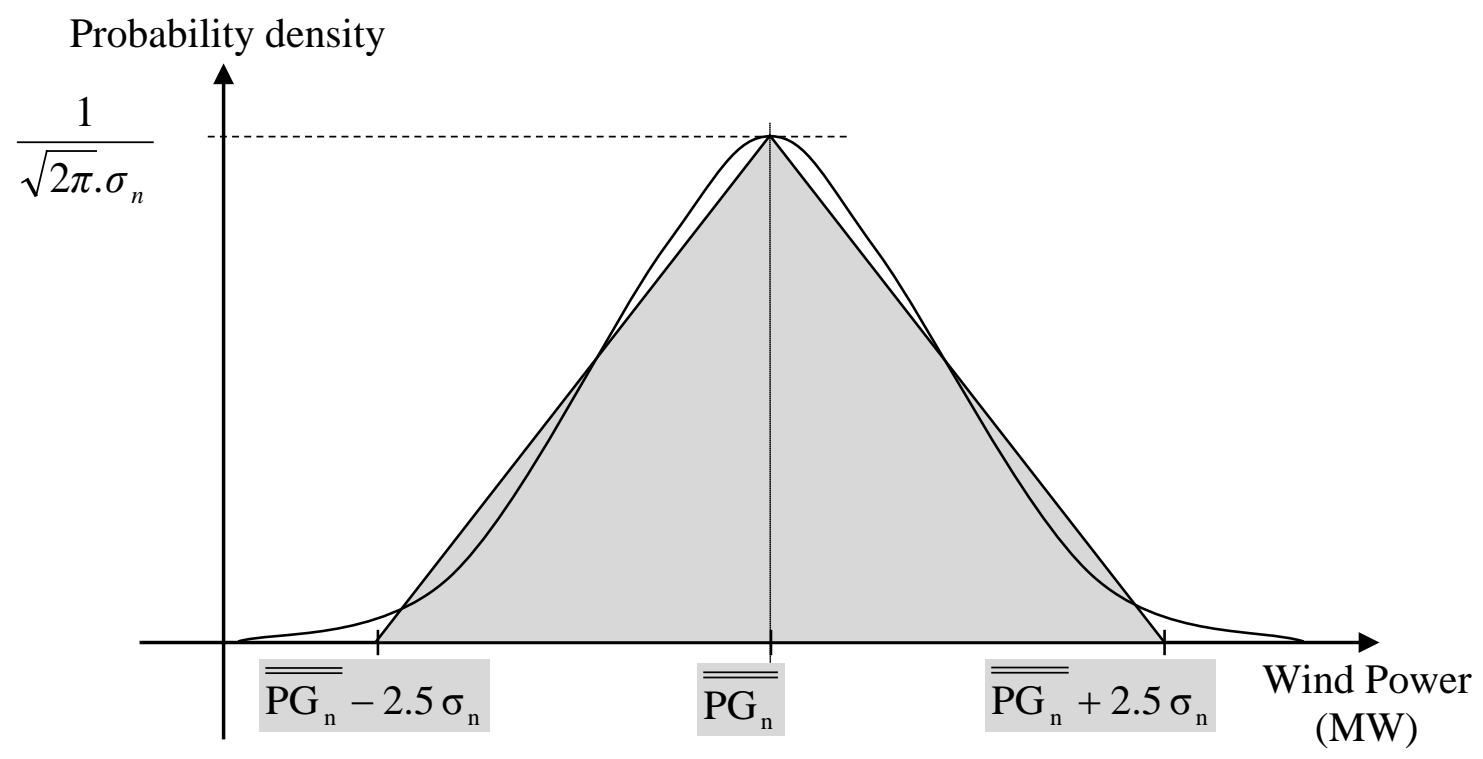

Fig. 2.1 TAD Model and the PDF curve of normal distribution 


\subsection{Probability calculation using the TAD model}

As shown in Figure 2.1, the TAD model is triangular. Its area is approximately equal to 1.0

for the interval from $\left(\overline{\overline{P G_{n}}}-2.5 \sigma_{n}\right)$ to $\left(\overline{\overline{P G_{n}}}+2.5 \sigma_{n}\right)$. This TAD model is used to develop an expression for the probability of wind power that quantifies unavailability. In order to develop an expression for the probability of WEGs, one can divide the TAD model into two parts, as shown in Figure 2.2.

Probability density

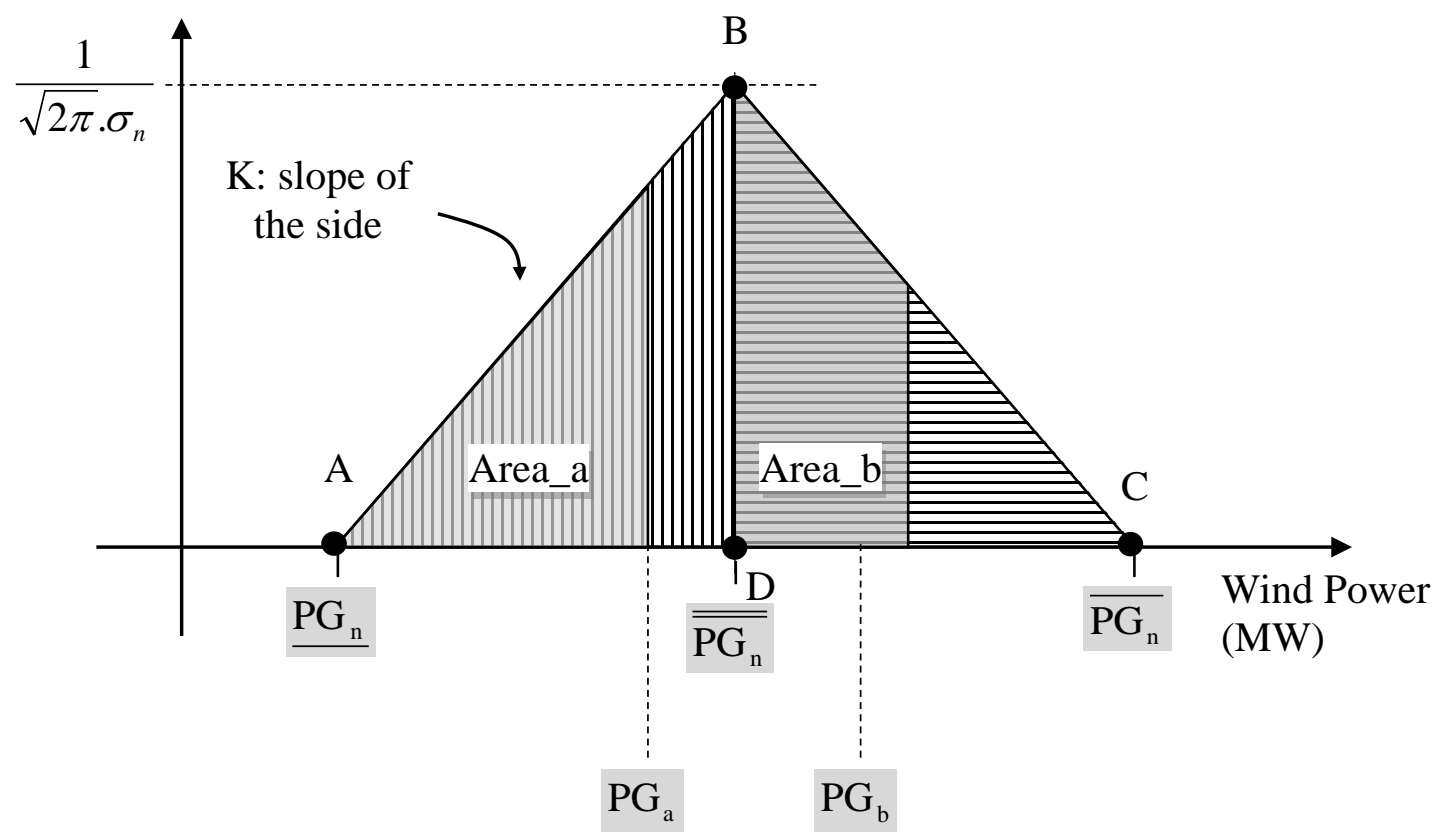

Fig. 2.2 Probability calculation of the TAD model 
The triangle $\mathrm{ABC}$ has two equal halves — named $\mathrm{ABD}$ and $\mathrm{BCD}$ - and each has an area of 0.5 . Using simple geometry, the following observations may be made:

The slope of the left side for the $n^{\text {th }}$ WEG is given by $K_{n}$ and it is equal to:

$K_{n}=\frac{1 / \sqrt{2 \pi}}{2.5 \sigma_{n}^{2}} \forall n \in W G$

Further, the minimum and maximum possible values of the $n^{\text {th }}$ WEG's power output in the

TAD model can be expressed as a function of the mean value $\left(\overline{\overline{P G_{n}}}\right)$ and standard deviations $\left(\sigma_{n}\right)$ as below:

$\underline{P G_{n}}=\overline{\overline{P G_{n}}}-2.5 \sigma_{n} \forall n \in W G \quad$ (minimum)

$\overline{P G_{n}}=\overline{\overline{P G_{n}}}+2.5 \sigma_{n} \forall n \in W G \quad$ (maximum)

The value of probability for a given wind power $\left(P G_{n}\right)$ of the $n^{\text {th }}$ WEG is equal to the area of the portion of the triangle with the base from $\underline{P G_{n}}$ to $P G_{n}$. There are two possibilities: one is when $P G_{n} \leq \overline{\overline{P G_{n}}}$ and the other is when $P G_{n}>\overline{\overline{P G_{n}}}$. The two cases are discussed as the following text.

1) $\underline{P G_{n}} \leq P G_{n} \leq \overline{\overline{P G_{n}}}$ : Consider an example: the WEG is being operated at a particular point of power output $\left(P G_{n}=P G_{a}\right)$, such that output of the WEG is less than the mean value ( $P G_{n} \leq \overline{\overline{P G_{n}}}$ ). In this case, the value of probability is equal to the area of the triangle on the left of $P G_{n}$ (shown in Figure 2.2). The probability is equal to the area of the shaded triangle on 
the left of $\mathrm{PG}_{\mathrm{a}}$ (Area_a) that is a part of the triangle ABD. The probability may be computed as:

$c p_{n}^{T}=\frac{1}{2} K_{n} \cdot\left(P G_{a}-\underline{P G_{n}}\right)^{2} \forall n \in W G$

When $P G_{n}$ is at the mean value ( $\overline{\overline{P G_{n}}}$ ) of the WEG, the probability is equal to 0.5 , which is the area of the triangle $\mathrm{ABD}$. In this case, the part of the right side triangle $\mathrm{BCD}$ that contributes to the value of the probability is zero.

2) $\overline{\overline{P G_{n}}}<P G_{n} \leq \overline{P G_{n}}$ : Consider another example of $P G_{n}=P G_{b}$, such that $P G_{n}>\overline{\overline{P G_{n}}}$. The probability contains areas of triangle $\mathrm{ABD}$ (equal to 0.5 ) and the trapezoid from $\overline{\overline{P G_{n}}}$ to $P G_{b}$ (Area_b). Thus, the value of the probability is equal to the area of the triangle ABC minus the triangle area on the right of $P G_{b}$ up to $\overline{P G_{n}}$. In this case, the value of the probability of the WEG may be computed using the following equation (2.8).

$c p_{n}^{T}=1-\frac{1}{2} K_{n} \cdot\left(\overline{P G_{n}}-P G_{b}\right)^{2} \forall n \in W G$

For example, consider a WEG with a forecasted power output (mean value, $\overline{\overline{P G}}$ ) equalling $50 \mathrm{MW}$ and a corresponding standard deviation $(\sigma)$ equalling 5 (10\% of $\overline{\overline{P G}}$ ). The value of probability of power output forecast from the WEG can be calculated by either integrating the PDF of normal probabilistic distribution or using the TAD model. The results are compared in Figure 2.3.

Equations (2.7) and (2.8) have been applied to this calculation process. Comparing two CDF 
curves, the TAD model gives an accurate estimate of the probability of the wind power output forecast. The probability values computed using the original PDF of normal distribution (dashed line) and the TAD model (solid line) are very close to each other. The absolute value of the maximum deviation between the CDF curves of the TAD model and the normal distribution is 0.02 . Taking advantage of this similarity, the TAD model can be used to develop an EENS function for WEGs. The method to compute EENS is presented in next section.

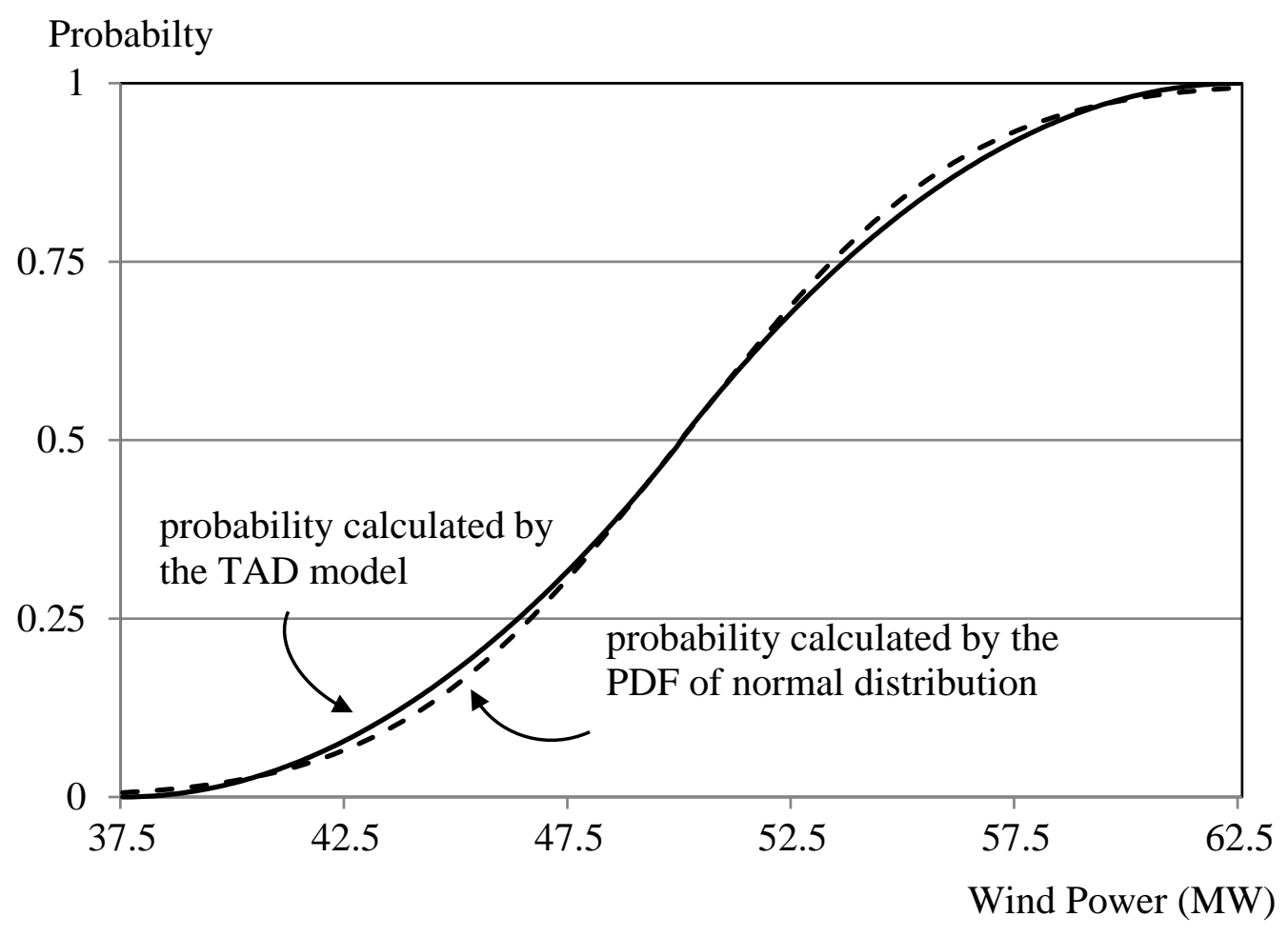

Fig. 2.3 Probability of wind power computed by normal distribution and the TAD model 


\subsection{Estimating EENS using the TAD model}

With given mean values $\left(\overline{\overline{P G_{n}}}\right)$ and standard deviations $\left(\sigma_{n}\right)$, the real power output from WEGs can be stochastically dispatched around their best forecasts. For a chosen output $P G_{n}$ from the $n^{\text {th }}$ WEG, the value of probability (computed using Equation 2.7 or 2.8) quantifies the probability that the $n^{\text {th }}$ WEG may not be able to supply power output $P G n$ into the grid. One can find out the EENS for the $n^{\text {th }}$ WEG as below.

By substituting (2.7) and (2.8) into equation (2.3), the EENS function can be written as the following patterns using the TAD model:

1). $\underline{P G_{n}} \leq P G_{n} \leq \overline{\overline{P G_{n}}}$

$E_{n}=\frac{1}{2} K_{n} \cdot\left(P G_{n}^{3}-2 \cdot \underline{P G_{n}} \cdot P G_{n}^{2}+\underline{P G_{n}^{2}} \cdot P G_{n}\right) \forall n \in W G$

2). $\overline{\overline{P G_{n}}}<P G_{n} \leq \overline{P G_{n}}$

$E_{n}=P G_{n}-\frac{1}{2} K_{n} \cdot\left(P G_{n}^{3}-2 \cdot \overline{P G_{n}} \cdot P G_{n}^{2}+\overline{P G_{n}^{2}} \cdot P G_{n}\right) \forall n \in W G$

One can observe from (2.9) and (2.10) that the EENS formulae of a WEG are functions of its real power output $\left(P G_{n}\right)$. Further, the value of standard deviation $\left(\sigma_{n}\right)$ helps to define the lower (2.5) and upper (2.6) bounds of wind power output in the TAD model. The effects of standard deviation are discussed further in the following section. 


\subsection{Influence of Wind Uncertainty on EENS value}

Depending on different forecasting conditions and operating points, the values of standard deviation or uncertainty can vary. The standard deviation affects the shape of wind power PDF and the corresponding EENS value. Figure 2.4 shows plots of EENS of a WEG versus power output computed with different standard deviation values.

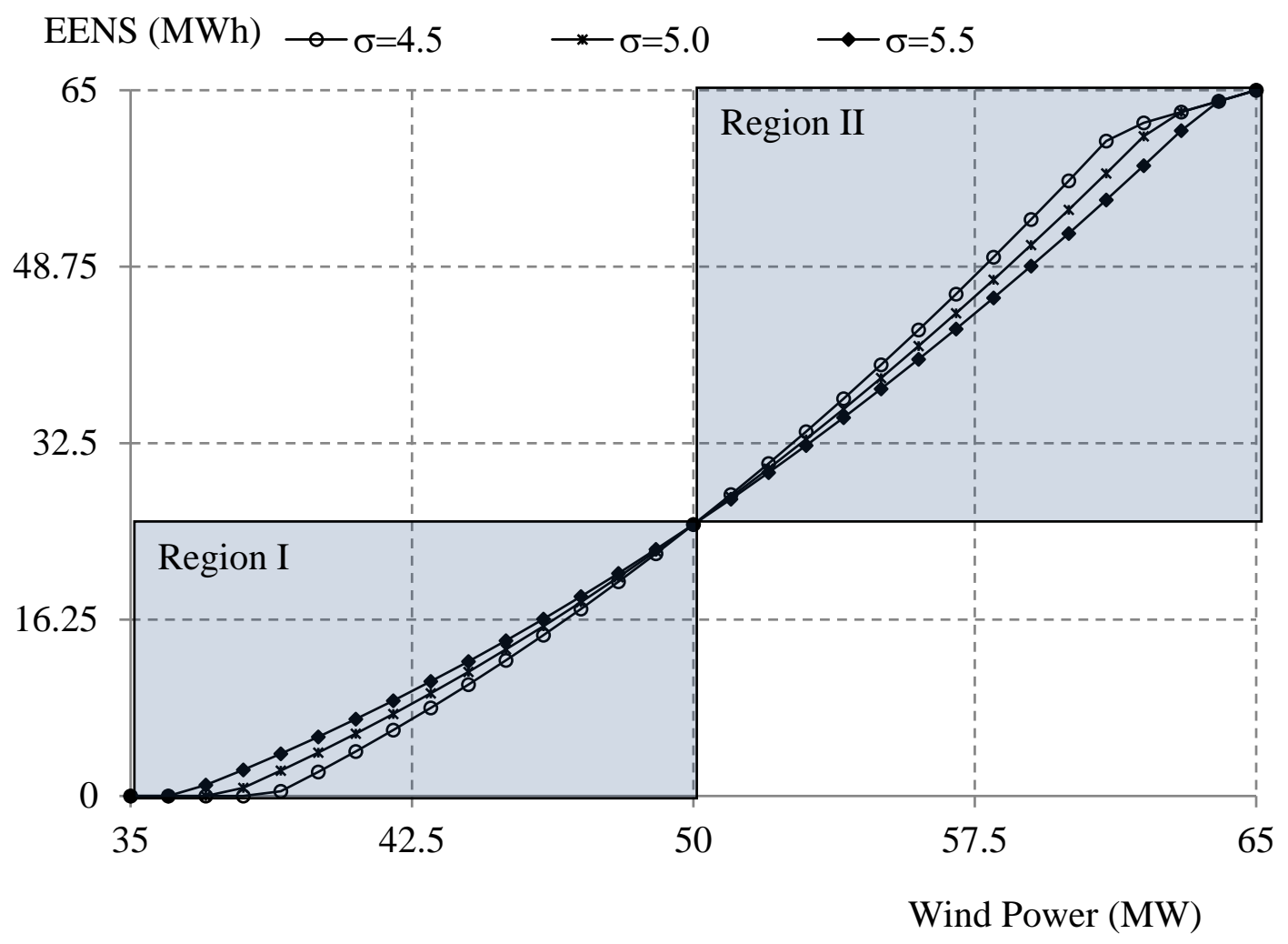

Fig. 2.4 EENS of wind power around the mean value (50MW) 
The expected wind power output is assumed to be $50 \mathrm{MW}$, and values of 4.5, 5.0, and 5.5 are used as various standard deviation values. The EENS value increases progressively with the value of standard deviation when the power output of the WEG is below its mean power output of $50 \mathrm{MW}$ (refer to Region I of Figure 2.4). On the contrary, when the output of WEG is greater than the mean value of $50 \mathrm{MW}$, EENS value decreases progressively with increasing values of standard deviation (see Region II of Figure 2.4). In this case, a larger value of standard deviation has a smaller EENS value.

Depending upon the value of EENS, system reserves need to be secured to compensate for the possible shortfall in generation, which ultimately depends upon the operating point and standard deviation of a wind power forecast. A numerical analysis of these values is given in next section.

\subsection{Case study on effects of standard deviation}

In order to demonstrate the influence of wind variability, a 2-unit system is created with one conventional generator (CEG) and one WEG. The energy price of the CEG is \$20.00/MWh and the spinning reserve cost is $\$ 10.40 / \mathrm{MWh}$. The expected power output (mean value) of the WEG is $50 \mathrm{MW}$ with a price of $\$ 2.00 / \mathrm{MWh}$. The total demand is $200 \mathrm{MW}$. Ten percent (10\%) of the demand and $60 \%$ of EENS quantify the risk and are compensated by the spinning reserve. Three standard deviation $(\sigma)$ values $(4.5,5.0$, and 5.5$)$ are used in the stochastic model of wind power output. Transmission security constraints are ignored for this case.

The objective of the optimization problem is to minimize the total operation cost (TC), which includes the cost of the CEG, the reserve cost, and the energy cost of the WEG. 
Minimize:

$\mathrm{TC}=\sum_{n \in C G}\left(b c_{n} \cdot P G_{n}+r c_{n} \cdot S R_{n}\right)+\sum_{n \in W G}\left(b w_{n} \cdot P G_{n}\right)$

Subject to the following two constraints:

1) Real power balance: $\sum_{n} P G_{n}=P D$

Sufficient real power $(P G)$ must be provided from both the CEG and the WEG to meet the electrical demand $(P D)$ in a particular time period of study (in this case, losses are ignored). Furthermore, the reserve is scheduled from the CEG to counter the risk of uncertainties leading to any shortfall of generation, as shown below.

2) Spinning reserve requirement as factors of total demand and EENS:

$$
\sum_{n \in C G} S R_{n} \geq \alpha \cdot \sum_{n \in W G} E_{n}+\beta \cdot P D
$$

Where $\alpha$ is equal to 0.6 , and $\beta$ is equal to 0.1 . The spinning reserve is scheduled to counter possible risks, including uncertainty of wind power (quantified by EENS) and possibility of any generator failure to supply the scheduled load (represented by partial demand). However, inter-temporal constraints are not considered in this case.

The formulation (2.11) to (2.13) is solved for various value of wind power output. A graph showing variations in the total cost versus wind power is shown in Figure 2.5. Since energy from the WEG is cheaper than that from the CEG, the total energy cost deceases when scheduling more power from the WEG. However, scheduling more power from the WEG 
increases reserve cost due to increasing EENS values. At system operating points where reserve cost is more expensive than generation costs, the total cost increases.

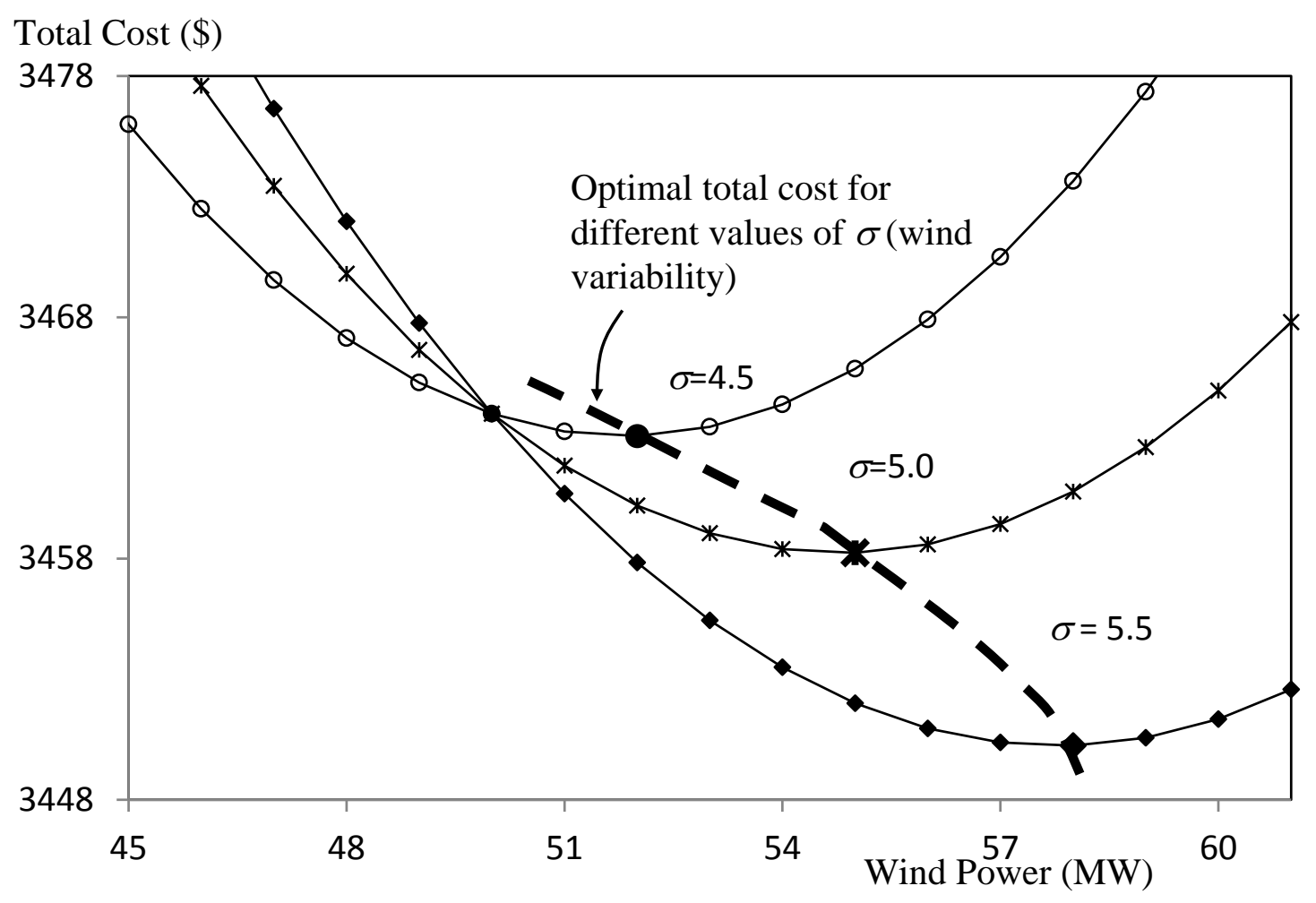

Fig. 2.5 Total cost changes with different $\sigma$ for the 2-unit system

Referring to Figure 2.4, it is shown that EENS decreases progressively with increasing values of standard deviation $(\sigma)$ when wind power output is greater than its mean value $(\overline{\overline{P G}})$. For the same amount of power from the WEG, a larger standard deviation value has less EENS. 
Further, a lower value of EENS requires lesser spinning reserve when accounting for shortcomings of the WEG.

In this case, with a larger standard deviation in its generation output, the system would have the capacity to consume more power from the WEG. Therefore, the total optimal cost decreases progressively with a larger standard deviation, as shown by the dashed line in Figure 2.5.

\subsection{Chapter Summary}

The function for EENS is nonlinear by nature. Incorporating this function would require constructing a nonlinear formulation of OPF and a mixed integer nonlinear formulation of dayahead UC.

Nonlinear formulations are computationally less robust than linear functions, especially for formulations including integer variables. It is therefore much more difficult to find the optimal solution for large practical systems using such nonlinear mixed integer formulations.

In order to overcome this difficulty, the nonlinear EENS function needs to be linearized for the OPF and day-ahead UC. The TAD model is therefore proposed as a highly accurate approximation of the nonlinear EENS function. The use of the TAD model for OPF applications are presented in the next chapter. 


\section{ChAPTER 3}

\section{A Practical Fast Probabilistic OPF Method}

\section{USING THE TAD MODEL}

Optimal power flow (OPF) algorithm is an important scheduling tool used in the daily operation of power systems. It minimizes the total operation cost (TC) while optimally scheduling power output of online generators. Additionally, near-real time OPF is used to optimally schedule reserve capacities from conventional electric generators (CEGs) to counter possible N-1 contingencies, which are referred as the failure of single equipment [70], and other uncertainties.

In this chapter, TAD model of WEGs is used to quantify EENS value due to wind generator output uncertainties and incorporated into a linearized formulation of OPF. Reserves in the OPF formulation are procured as a function of $\mathrm{N}-1$ contingency requirement in addition to a fraction of EENS value computed through the TAD model. This proposed OPF formulation is solved using the successive linear programming (SLP) technique. Further, case studies are reported and their results are discussed. 


\subsection{Problem formulation of fast Optimal Power Flow}

The objective of the fast OPF algorithm is to minimize the total cost (TC) of real power generation while satisfying bus-wise power balance equations, limits on generator capacities, limits on bus voltage magnitudes, etc. The term TC includes the cost of energy from CEGs and WEGs and the cost of spinning reserves. This work assumes that WEGs bid into the electricity market. Reserve is purchased in OPF algorithm at a lower rate than scheduled real power generation.

Therefore, by including the cost of wind energy, the TC for the fast OPF algorithm can be formulated as below to determine the optimal generation schedule. The objective is to minimize:

$\mathrm{TC}=\sum_{n \in C G}\left[a c_{n}+\sum_{m}\left(b c_{n m} \cdot P M_{n m}\right)+r c_{n} \cdot S R_{n}\right]+\sum_{n \in W G}\left(a w_{n}+b w_{n} \cdot P G_{n}\right)$

In the cost function (3.1), $a c_{n}(\$)$ is the fixed cost for the $n^{\text {th }}$ CEG; $b c_{n m}(\$ / M W h)$ is the incremental energy price for CEGs; and $r c_{n}(\$ / M W h)$ is the price of reserved energy supplied by CEGs. WEGs also have fixed costs of $a w_{n}(\$)$ and incremental energy prices of $b w_{n}(\$ / M W h)$ respectively. Minimization of the objective function is subject to the following constraints:

\section{Power balance constraints:}

Real power balance: $\quad \sum_{n \in N I_{i}} P G_{n}=P D_{i}+P T_{i}(\delta, V) \forall i$

Reactive power balance: $\sum_{n \in N I_{i}} Q G_{n}=Q D_{i}+Q T_{i}(\delta, V) \forall i$ 
The power balance equations (3.2) and (3.3) ensure that the OPF algorithm can schedule sufficient real and reactive power to meet all real-time demands. $P T_{i}(\delta, V)$ and $Q T_{i}(\delta, V)$ are functions of voltage magnitudes and phase angles that equal the total real and reactive powers flowing from the $i^{\text {th }}$ bus to all connected transmission lines and transformers respectively (as detailed as equations A.1 and A.2 in Appendix A). In addition, real and reactive power outputs of generators are limited by the physical capacities as formulated in equations (3.4), (3.5), (3.6), and (3.7).

\section{Generator real power output limit:}

Real power in segments (CEGs only): $\quad 0 \leq P M_{n m} \leq \overline{P M_{n m}} \forall n \in C G, m$

$$
P G_{n}=\sum_{m} P M_{n m} \forall n \in C G
$$

In equations (3.4) and (3.5), real power generation of each CEG comprises of ' $M$ ' segments. Energy costs of segments are assumed to be increasing where the first segment costs the least and the $M^{\text {th }}$ segment costs the most. Thus, power in every segment $\left(P M_{n m}\right)$ is bounded by its segment capacity. These linear cost coefficients of segments constitute piece-wise cost function of a generator that it is amendable to a LP solver. The total generation of CEGs can be computed by summing up segment-wise power output of all generators. Real and reactive powers are limited in ranges according to unit capacities.

Real power in units (CEGs and WEGs): $\quad P G_{n} \leq P G_{n} \leq \overline{P G_{n}} \quad \forall n$

Generator reactive power output limit: $\quad \underline{Q G_{n}} \leq Q G_{n} \leq \overline{Q G_{n}} \quad \forall n \in C G$ 
Spinning reserves must be scheduled from CEGs to ensure stability of system operation.

\section{Spinning reserve constraints:}

Spinning reserve requirement: $\quad \sum_{n \in C G} S R_{n} \geq M R$

In (3.8), $M R$ is the minimum system reserve requirement, which is decided by the system's operational philosophy. For the $\mathrm{N}-1$ contingency criterion, the value of $M R$ is determined by the capacity of largest online generation units [59]. The spinning reserve supplied by a particular CEG is limited by its generation capacity, scheduled generation and 10 minutes ramping speed ( $R 10)$.

Spinning reserve capacity: $S R_{n} \leq \min \left\{R 10_{n}, \overline{P G_{n}}-P G_{n}\right\} \forall n \in C G$

Spinning reserve criteria: $\quad \sum_{n \in C G} S R_{n} \geq \alpha \cdot \sum_{n \in W G} E_{n}+\beta \cdot \sum_{i} P D_{i}$

Shortfall in real power from potential failure of any generator is compensated by spinning reserve. The total cost (3.1) is influenced by the uncertainty of power output of WEGs in terms of their EENS values. To quantify the overall possible risks of generation shortfall, a fraction $(\alpha)$ of EENS value (due to uncertainty of supply from WEGs) and a fraction $(\beta)$ of the total system hourly demand $\sum P D_{i}$ (due to equipment unreliability) are accounted for in equation (3.10).

In order to study the effect of output uncertainty of WEGs, $\alpha$ is altered while $\beta$ is kept constant at $10 \%$. The maximum available wind power generation is estimated through wind forecasts and a portion is scheduled by weighing their benefits with reserve costs required to 
counter their EENS values [6]. The EENS of a given power output of a WEG can be computed using (2.3) in Chapter 2. Furthermore, the fast OPF is constrained by AC transmission security constraints.

Line and Transformer power flow MVA limit: $0 \leq S F_{l} \leq \overline{S F_{l}} \quad \forall l$

Transmission line and transformer power flows are constrained by their MVA limits. The MVA line and transformer flow $\left(S F_{l}\right)$ is a function of bus voltage phasors of the buses connected to both ends of the $l^{\text {th }}$ line or transformer (as detailed in Appendix A). Both directions of line/transformer flows are considered in (3.11). Bus voltage magnitudes and phase angles are also constrained for all buses in the system.

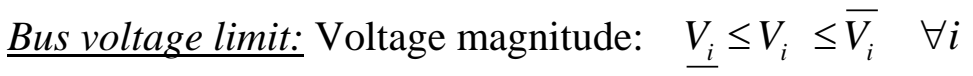

$$
\text { Voltage phase angle: }-\pi \leq \delta_{i} \leq \pi \quad \forall i
$$

In the above formulation from (3.1) to (3.13), EENS function, power balance equations, and the MVA line/transformer power flow constraints are nonlinear. Accordingly, the SLP technique is used to solve this nonlinear optimization challenge presented in (3.1) to (3.13). The fast OPF formulation is linearized around the current operating point $\left(X^{0}\right)$. This linearized formulation, as presented in the next section, is optimized to find the optimal changes in the decision vector $\Delta X$ to minimize the corresponding change in the total operating cost $\Delta \mathrm{TC}$. The decision vector of the linearized formulation $\Delta X=[\Delta P G \Delta S R \Delta V G]$, which are changes in real power generation, spinning reserve, and voltage magnitudes of generator buses. The continuous variables are updated with these optimal changes $\left[X=X^{0}+\Delta X\right]$. Using the update 
decision vector $X$, the state of the power system $(V, \delta)$ is estimated by solving bus-wise power balance equations. This process of linearizing, solving the LP formulation, updating the state and solution of power balance equations is repeated several times until the optimal solution is found. The linearized formulations of EENS function and the fast OPF are shown in next section.

\subsection{Linear formulation of EENS and fast OPF}

As discussed in Chapter 2, the formulation of EENS function (2.3) using the normal probabilistic distribution is a nonlinear exponential function of real power output of WEGs. The exponential function of EENS is difficult to incorporate into the formulation of the fast OPF for either online or offline optimization. However, taking advantage of geometric similarity, the TAD model can accurately model the normal probabilistic distribution of WEGs output forecast, (2.9) and (2.10).

\subsubsection{Linearized function of EENS using the TAD model}

Applying Taylor's expansion and reducing (2.3) to a first order expression, the incremental EENS function can be written as below:

$\Delta E_{n}=J E P_{n} \cdot \Delta P G_{n} \forall n \in W G$

The sensitivity matrix, which relates change in the power output of WEGs to change in corresponding EENS, is derived below by considering the two cases in Chapter 2: 
1). $\underline{P G_{n}}<P G_{n}^{0} \leq \overline{\overline{P G_{n}}}$

$J E P_{n}=\frac{d E_{n}}{d P G_{n}}=\frac{1}{2} K_{n} \cdot\left[3\left(P G_{n}^{0}\right)^{2}-4 \cdot \underline{P G_{n}} \cdot P G_{n}^{0}+\underline{P G_{n}^{2}}\right]$

2). $\overline{\overline{P G_{n}}}<P G_{n}^{0} \leq \overline{P G_{n}}$

$$
J E P_{n}=\frac{d E_{n}}{d P G_{n}}=1-\frac{1}{2} K_{n} \cdot\left[3\left(P G_{n}^{0}\right)^{2}-4 \cdot \overline{P G_{n}} \cdot P G_{n}^{0}+\overline{P G_{n}^{2}}\right]
$$

Depending upon the current power output $\left(P G_{n}^{0}\right)$ of a WEG, the value of the sensitivity matrix $\left(J E P_{n}\right)$ is estimated by using either (3.15) or (3.16). The linear incremental function of EENS is incorporated into the linearized fast OPF formulation. The complete formulation is given in the following section.

\subsubsection{Complete formulation of fast probabilistic OPF with the TAD model}

Consider the current operating point $X^{0}$. The aim is to determine optimal change $\Delta X$ to minimize change in total cost ( $\Delta \mathrm{TC})$. Using (3.1) to (3.13) and changing continuous variables in to their incremental forms, one gets an incremental LP formulation as the following:

Minimize $\Delta \mathrm{TC}$ in terms of incremental variables, $\Delta X=[\Delta P G \Delta S R \Delta V G]$ :

$\Delta \mathrm{TC}=\sum_{n \in C G}\left[\sum_{m}\left(b c_{n m} \cdot \Delta P G_{n m}\right)+r c_{n} \cdot \Delta S R_{n}\right]+\sum_{n \in W G} b w_{n} \cdot \Delta P G_{n}$

Optimization of this objective is subject to the following linear constraints: 


\section{Power balance equations:}

Incremental real power balance equation: $\quad \sum_{n \in N I_{i}} \Delta P G_{n}=J P D . \Delta \delta+J P V . \Delta V \forall i$

Incremental reactive power balance equation: $\sum_{n \in N I_{i}} \Delta Q G_{n}=J Q D . \Delta \delta+J Q V . \Delta V \forall i$

$J P D, J P V, J Q D$, and $J Q V$ are sub-matrices of the transmission system Jacobian matrix. They are derivatives of real and reactive power with respect to voltage phase angles and magnitudes. The Jacobian matrix is evaluated at the current voltage phasor values $\left(\left[V^{0}, \delta^{0}\right]\right)$ in each iteration. All linear and nonlinear constraints are transformed into incremental linear constraints.

\section{Generator real power output limit:}

Real power in segments (CEGs only): $\quad 0 \leq P M_{n m}^{0}+\Delta P M_{n m} \leq \overline{P M_{n m}} \forall n \in C G, m$

$$
\Delta P G_{n}=\sum_{m} \Delta P M_{n m} \forall n \in C G
$$

Real power in units: $\quad \underline{P G_{n}} \leq P G_{n}^{0}+\Delta P G_{n} \leq \overline{P G_{n}} \forall n$

Generator reactive power output limit:

$\underline{Q G_{n}} \leq Q G_{n}^{0}+\Delta Q G_{n} \leq \overline{Q G_{n}} \forall n \in C G$ 


\section{Spinning reserve constraints:}

Spinning reserve capacity:

$S R_{n}^{0}+\Delta S R_{n} \leq \min \left\{S R 10_{n}, \overline{P G_{n}}-\sum_{m}\left(P G_{n m}^{0}+\Delta P G_{n m}\right)\right\} \forall n \in C G$

Spinning reserve requirement: $\quad \sum_{n \in C G}\left(S R_{n}^{0}+\Delta S R_{n}\right) \geq M R$

Reserve criteria: $\quad \sum_{n \in C G}\left(R S_{n}^{0}+\Delta R S_{n}\right) \geq \alpha \cdot \sum_{n \in W G}\left(E_{n}^{0}+\Delta E_{n}\right)+\beta \cdot \sum_{i} P D_{i}$

Line power flow limit: $\quad 0 \leq S F_{l}^{0}+\Delta S F_{l} \leq \overline{S F_{l}} \forall l$

Bus voltage limit:

Voltage magnitude: $\quad \underline{V_{i}} \leq V_{i}^{0}+\Delta V_{i} \leq \bar{V}_{i} \forall i$

Voltage phase angle: $\quad-\pi \leq \delta_{i}^{0}+\Delta \delta_{i} \leq \pi \forall i$

Control variables, $\Delta P G$ (for all generators) and $\Delta V G$ (for conventional generators only), are bounded within a narrow range to ensure that the linear model is valid. Accordingly, limits are imposed on the control variables as below:

$$
\begin{aligned}
& -\overline{P G_{n}} \leq \Delta P G_{n} \leq+\overline{\overline{P G_{n}}} \quad \forall n \\
& -\overline{V G_{n}} \leq \Delta V G_{n} \leq+\overline{\overline{V G_{n}}} \quad \forall n \in C G
\end{aligned}
$$


The LP model of the fast OPF (3.17) to (3.31) is set up at current operating point $X^{0}$ and solved yielding the optimal change in decision vector $\Delta X$. On updating the decision variables $X=X^{0}+\Delta X$, power balance equations are solved to determine the state of the system $(\delta, V)$ using the Newton Raphson technique. Locational marginal prices (LMP) at all buses can be obtained by dual variables corresponding to power balance equations in the incremental LP formulation. The complete algorithm of the proposed fast OPF method that incorporates the TAD model of WEGs is presented in the following section.

\subsection{Algorithm of fast OPF with the TAD model of WEGs}

The steps used by the proposed fast OPF algorithm are same as that of SLP. The algorithm aims to solve the problem defined in (3.1) to (3.13). The algorithm uses a starting state as the current state $X^{0}$ to set up and compute an incremental model (3.17) to (3.31). This model is solved to determine optimal changes in the incremental decision vector $\Delta X$. The continuous decision vector $\left(X=X^{0}+\Delta X\right)$ is updated and AC power balance equations are solved to determine the state of the power systems $(V, \delta)$. The steps of setting up of LP model, its solution, updating the decision vector and determination of the state are a set of steps that are successively repeated until the optimal solution is reached.

An explanatory flowchart for the algorithm is presented in Figure 3.1. The detailed steps are shown below. 


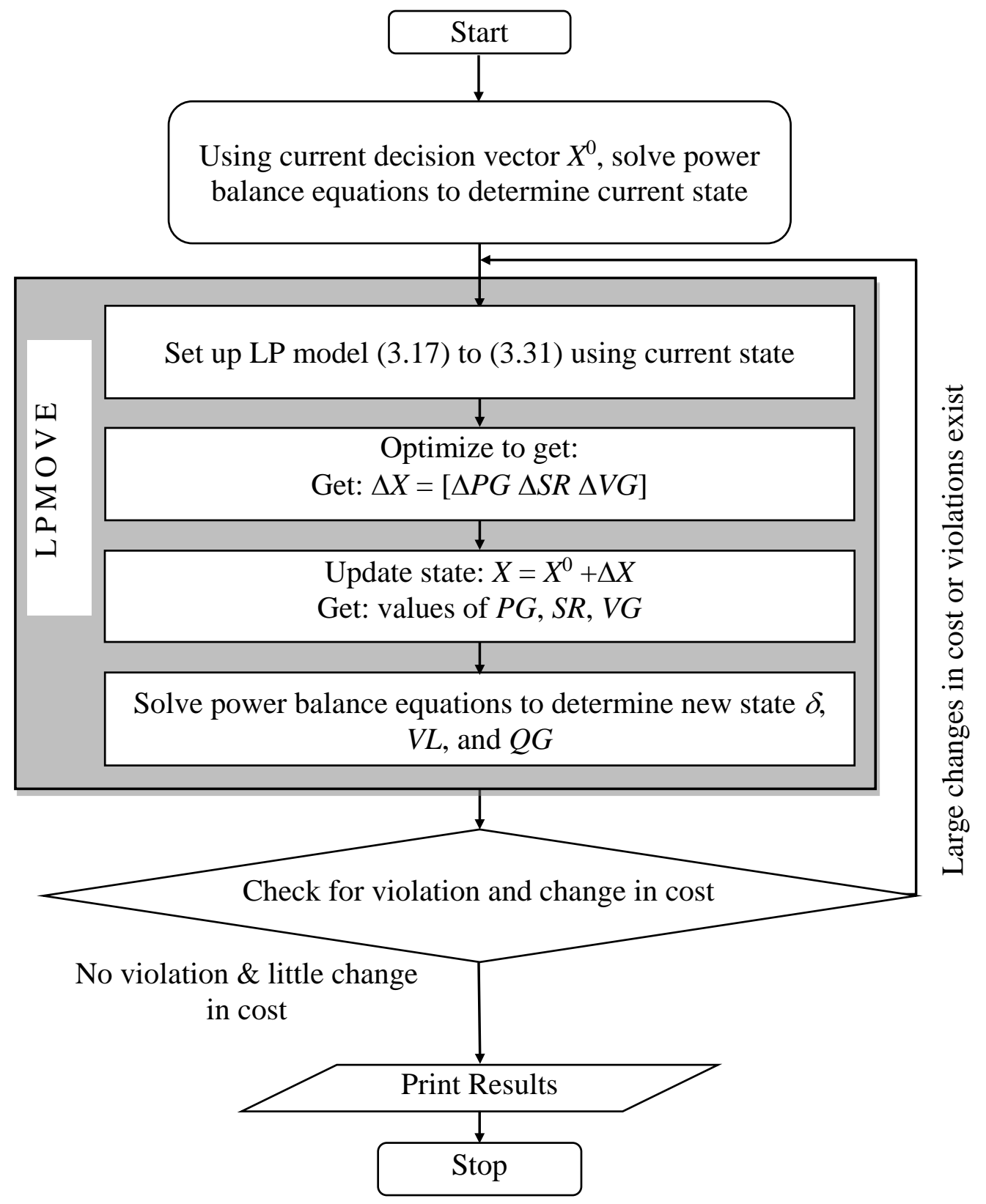

Fig. 3.1 Flowchart of proposed OPF method with TAD model 
Step 1: Solve AC power balance equations using the current state $X^{0}$.

Step 2: Set up an incremental LP model, (3.17) to (3.31), by using the proposed TAD model of WEGs.

Step 3: Solve the incremental LP model, (3.17) to (3.31), to determine the optimal changes in $X: \Delta X=[\Delta P G \Delta S R \Delta V G]$.

Step 4: Update $X=[P G S R V G]$.

Step 5: Solve the AC power balance equations to determine the updated state $(\delta, V L, Q G)$.

Step 6: Repeat steps 2 to 5 until violations are removed and $\Delta \mathrm{TC}$ is insignificant.

The set of steps 2 to 5 constitute one LPMOVE. This set is repeated until the constrained optimal solution is reached. To test this solution, two cases are studied using the proposed fast OPF with the TAD model in the next section.

\subsection{Test results and discussions}

A modified IEEE 30-bus system and a modified IEEE 118-bus system are used to test the proposed fast OPF algorithm with the TAD model. The details are given in the following sections.

\subsubsection{Modified IEEE 30-bus System}

The transmission network and load data of the IEEE 30-bus system can be found in [60]. The price data of CEGs is given in Appendix B. The system has 6 generators on buses \#1, \#2, 
$\# 5$, \#8, \#11, and \#13. Three WEGs are installed on buses \#26, \#29, and \#30 for testing purposes. The expected power outputs (mean values) of the three WEGs are $20 \mathrm{MW}, 30 \mathrm{MW}$, and 50 MW respectively. The value of standard deviation is assumed as $10 \%$ of the expected power outputs; a value commonly adopted by many researchers [10] and [47].

The use of the SLP method to implement the fast OPF algorithm with the TAD model is compared to the classical enumeration technique. The enumeration technique is applied on the nonlinear formulation of (3.1) to (3.13) to obtain the optimal working point of the system. In the enumeration method, the PDF of each WEG is divided into 10 segments. Each segment has an expected output and a corresponding probability of occurrence. Considering all possible combinations of outputs from the three WEGs, 1,000 scenarios are created. One thousand EENS values, which cover all possible cases for the IEEE 30-bus system with three WEGs, are computed separately by solving (3.1) to (3.13). By solving the nonlinear formulation 1,000 times corresponding to each scenario, one can find the scenario with the minimum total cost among these 1,000 scenarios, shown in Figure 3.2.

This minimum cost scenario and its associated LMPs will be used to verify the result of the proposed fast OPF with the TAD model of WEGs. The algorithm for the enumeration method is given in Appendix A. The spinning reserve cost of a CEG is assumed as $15 \%$ of the energy bidding prices in the highest power output segment. In this simulation, the value of $\alpha$ is equal to 0.6 . 


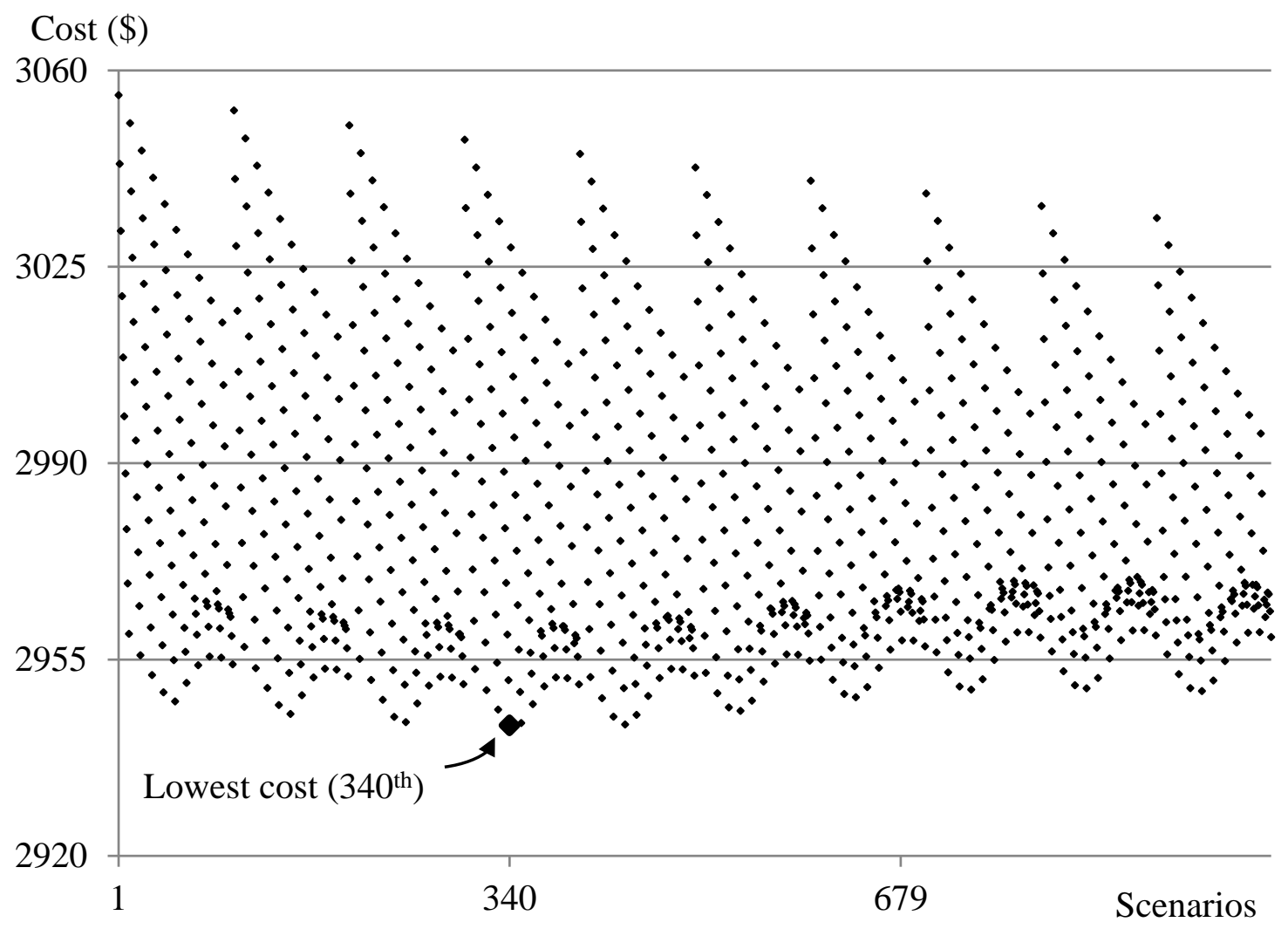

Fig. 3.2 Costs of 1000 scenarios of 30-bus system

The total operating cost for the system was calculated for 1,000 scenarios generated by the enumeration method. This process of computing 1,000 scenarios considering only three WEGs took 893.18 seconds (close to 15 minutes). This execution time will increase exponentially with additional WEGs. The lowest cost appeared at the $340^{\text {th }}$ scenario. The solution with the least cost is used to verify the proposed method.

The proposed fast OPF algorithm with the TAD model of WEGs took only 7 LPMOVEs consuming 2.05 seconds of computational time to solve the same IEEE 30-bus system and 
reach the optimal solution. This optimal solution is very close to the least cost scenario obtained by the enumeration method as shown in Table 3.1. It is noted that the reserve requirement without considering EENS is 56.58 MW (3.10). The final total costs and EENS are nearly identical for both of the methods. It must be noted that the computation time for the proposed algorithm with the TAD model of WEGs shall increase only marginally when increasing the number of WEGs.

Table 3.1 Total Costs and Execution Times of Two Methods for the 30-Bus System

\begin{tabular}{|c|c|c|c|c|c|}
\hline \multirow{2}{*}{ Method } & Cost & EENS & $\begin{array}{c}\text { Scenarios [Min cost] - } \\
\text { enumeration } \\
(\$)\end{array}$ & $\begin{array}{c}\text { Execution time } \\
\text { (MWh) }\end{array}$ & $\begin{array}{c}\text { Reserve } \\
\text { (SPMOVEs - TAD }\end{array}$ \\
\hline Enumeration & $2,943.27$ & 71.39 & $1,000[340]$ & 893.18 & 99.41 \\
\hline TAD & $2,935.84$ & 73.96 & 7 & 2.05 & 100.96 \\
\hline
\end{tabular}

Note: Reserve requirement without EENS is 56.58 MW (3.10)

Figure 3.3 shows the LMPs of real power at all buses in the IEEE 30-bus system. The result of the $340^{\text {th }}$ scenario of the enumeration technique is compared with the results obtained using the proposed fast OPF algorithm with TAD model. This graph demonstrates that the proposed fast OPF algorithm with the TAD model of WEGs is accurate. 


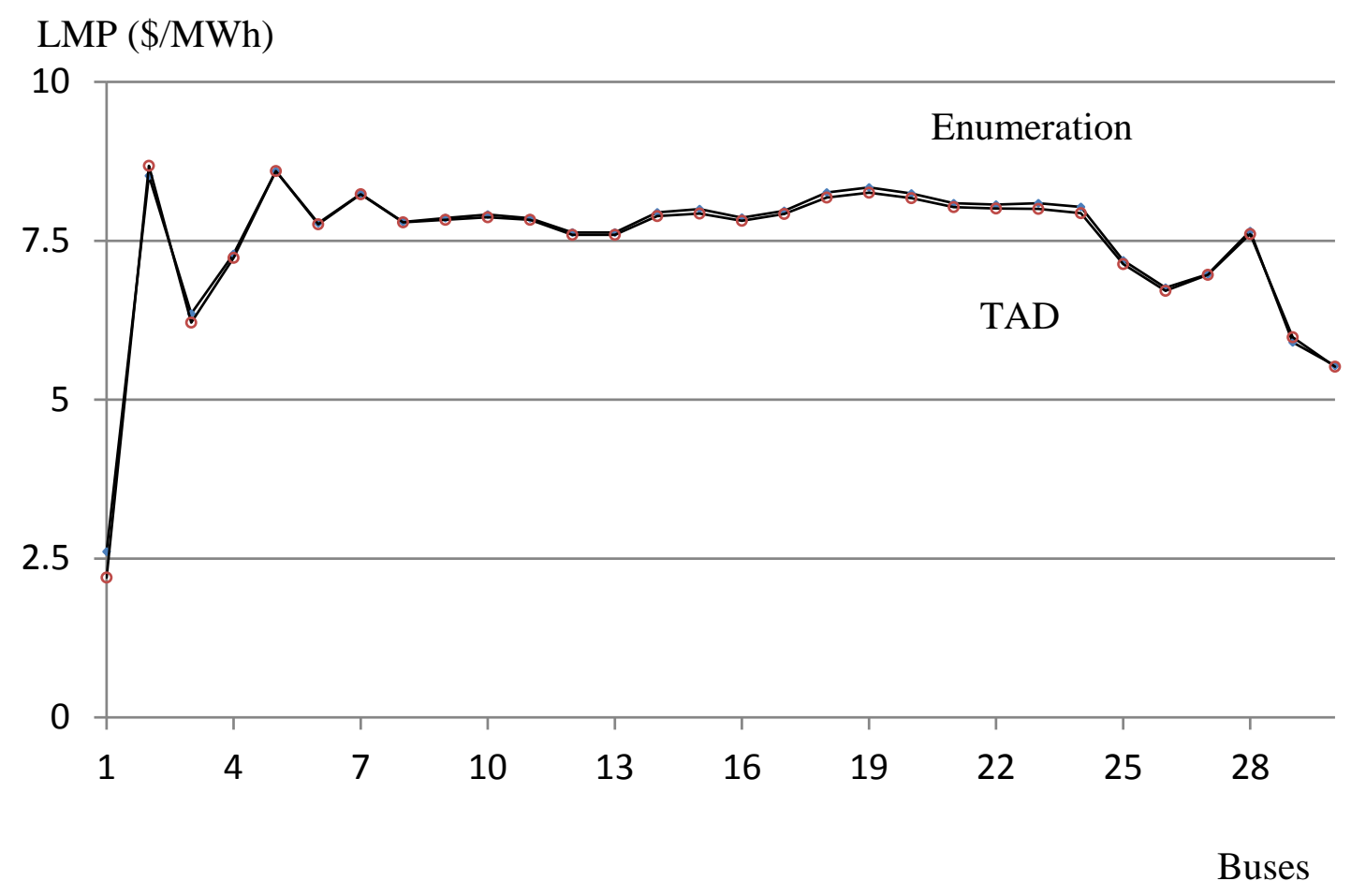

Fig. 3.3 Comparison of real power LMPs at all buses of the 30-bus system

Figures 3.4 and 3.5 show the effects of varying the value of $\alpha$ on the total operating cost, the output of WEGs, the reserve cost, and total EENS value. Referring to (3.10), one can see that the factor $\alpha$ is the portion of EENS value carried as spinning reserve by the system, and that EENS value depends upon the total output from the WEGs in the system. As the value of $\alpha$ increases for a given WEG output, a higher amount of reserve is required to counter the same EENS, which would raise the total costs (3.1). To address this rise in costs, the fast OPF algorithm minimizes the total costs by optimally scheduling WEGs while countering the cost of risk due to their output uncertainty, quantified by EENS. 


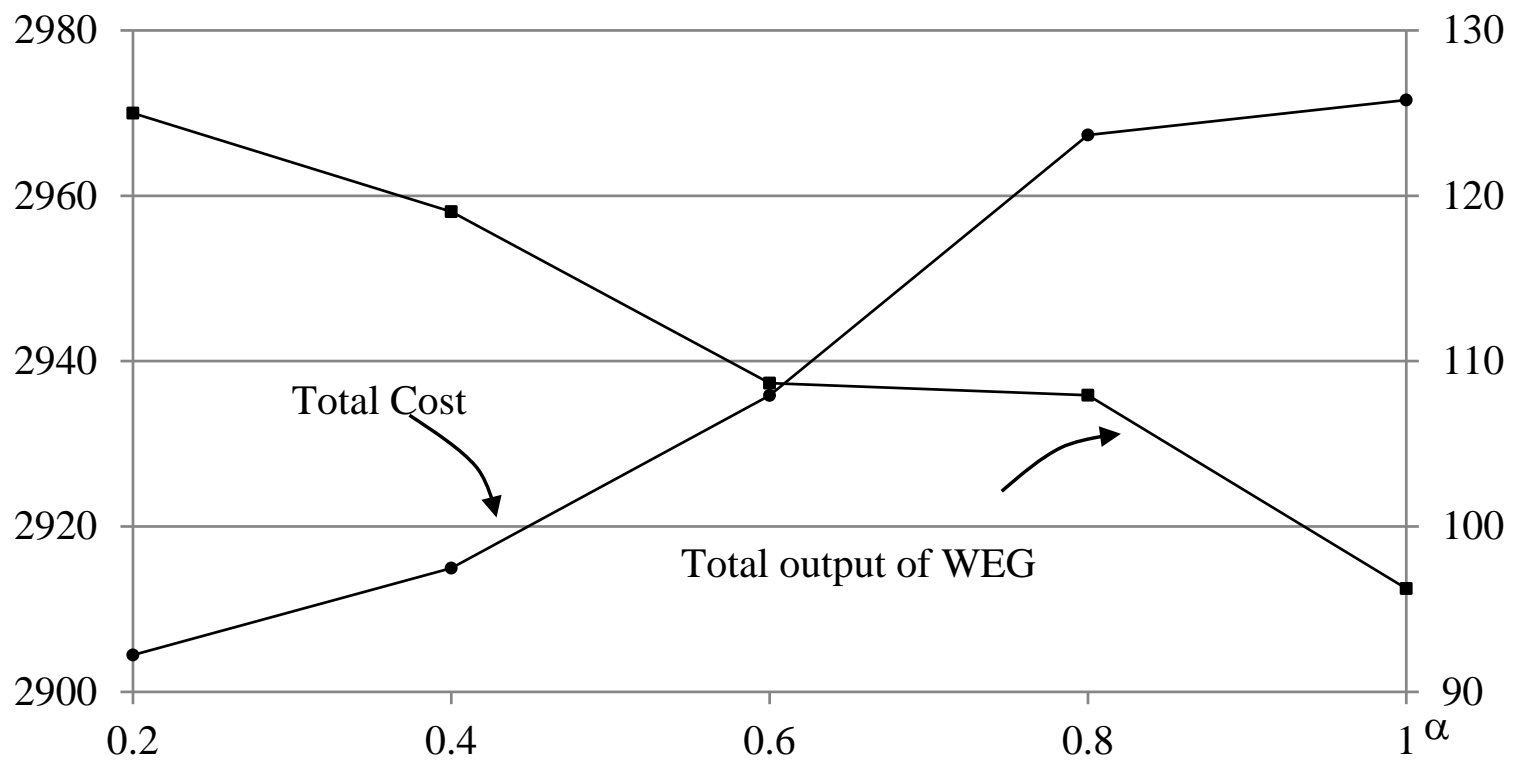

Fig. 3.4 Total cost and output of WEGs with different $\alpha$ for the 30-bus system

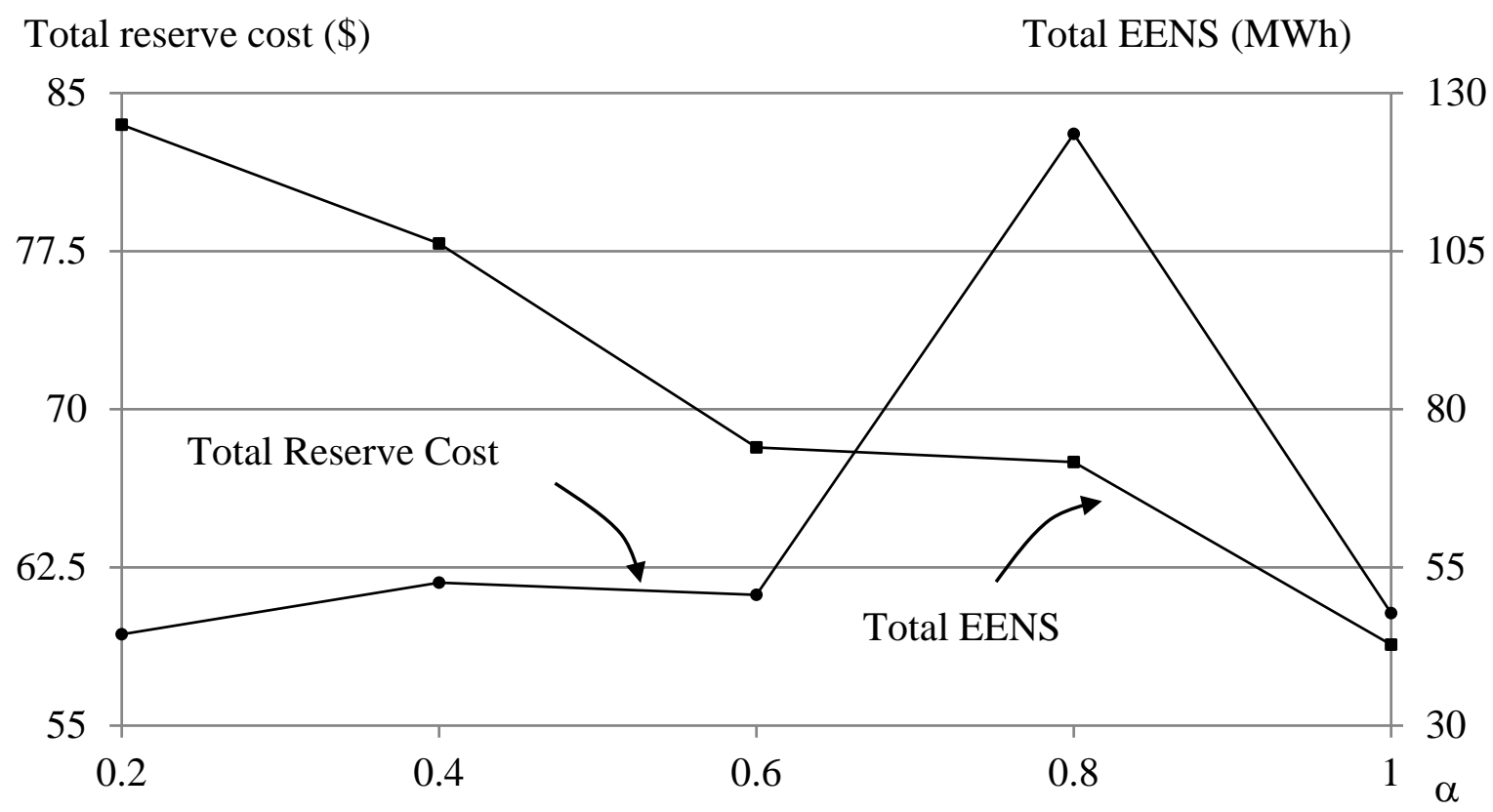

Fig. 3.5 Reserve cost and EENS with different $\alpha$ for the 30-bus system 
Reserve cost is affected not only by values of EENS (from WEGs) but also the reserve providers (CEGs). With a decrease of wind power generation, CEGs must supply more power to meet the unchanged demand. This causes cheap CEGs losing reserve capacity. Reserves are scheduled from expensive generators, and reserve cost increases. On the contrary, when wind generation reduces, the amount of reserve reduces, as well as the total reserve cost. These conflicting actions explain why reserve cost increases to a peak and then reduces as the value of $\alpha$ increases, shown in Figure 3.5.

For the proposed fast OPF method with the TAD model of WEGs, it takes only a few seconds to complete the analysis and produce the graph in Figures 3.4 and 3.5. In contrast, using the enumeration technique would require more than one hour to collect the results necessary to produce graphs of Figures 3.4 and 3.5. This advantage in computational time highlights the practical online applicability of the proposed fast OPF method with the TAD model of WEGs. The application of the proposed method for a larger system is given in next section.

\subsubsection{IEEE 118-bus System}

The modified IEEE 118-bus system is used to demonstrate the applicability of the proposed fast OPF algorithm with the TAD model of WEGs for larger systems. The detailed transmission network, load, and bidding information of this IEEE 118-bus system can be found in [46]. The costs of CEGs are linearized to fit the LP formulation, as detailed in Appendix B. Three conventional units are replaced by WEGs on buses \#10, \#80, and \#89 for testing

purposes. The expected power outputs of the three WEGs are all given as 300 MW with standard deviations of 30 . 
Similar to what was done for the IEEE 30-bus system, one thousand $(1,000)$ scenarios were generated based on the outputs of the three installed WEGs, and sixty percent of EENS is carried by reserves $(\alpha=0.6)$. The lowest cost appears at the 994th scenario, shown in Figure 3.6.

Cost (\$)

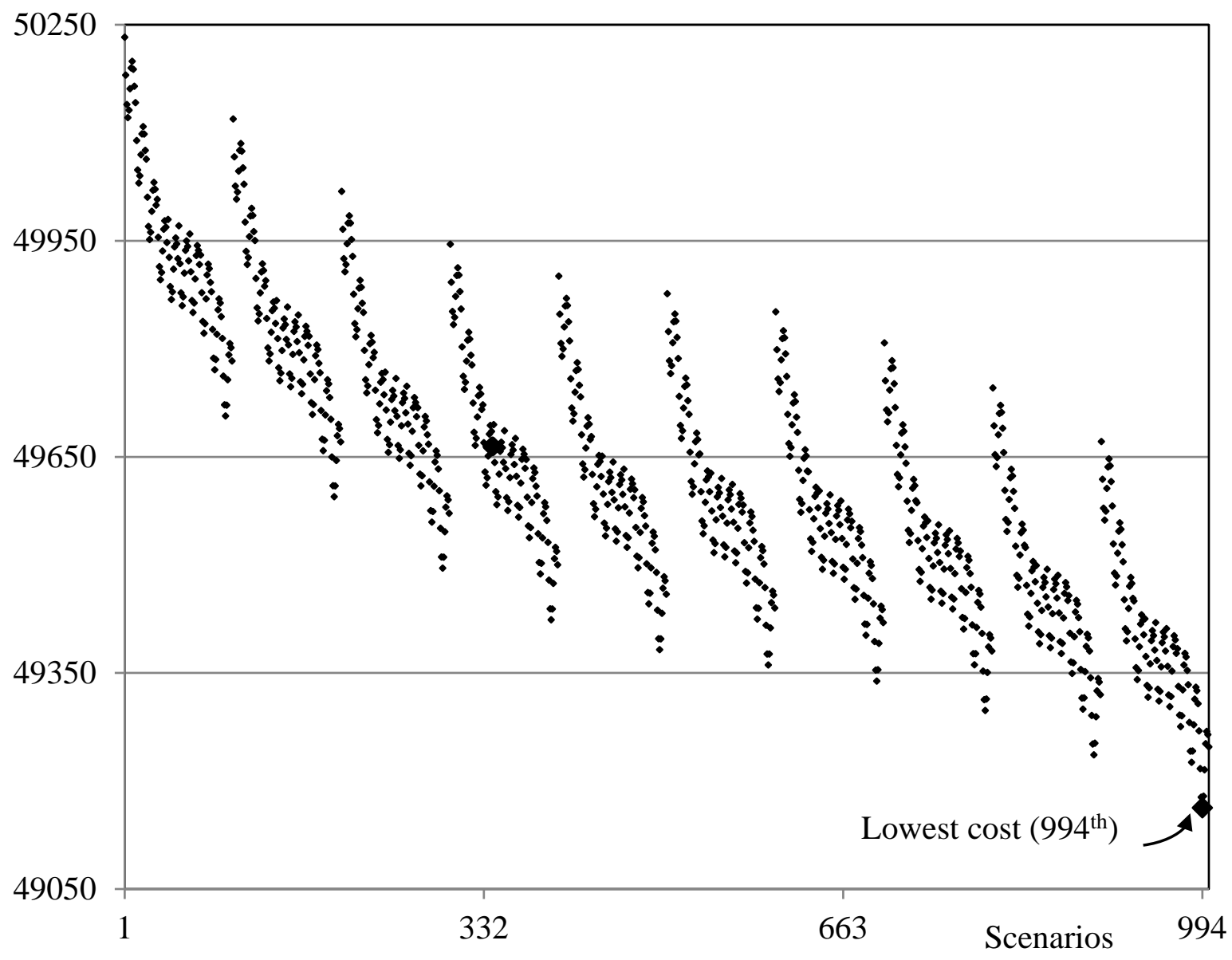

Fig. 3.6 Costs of 1000 scenarios of 118-bus system 
Table 3.2 presents and compares the minimum costs and execution times of these two methods. The least cost also appears in the $994^{\text {th }}$ scenario of the enumeration method, which requires a significant amount time to achieve. The execution time of the fast OPF with the TAD model is far less than that of the enumeration method. The expected reserve without considering EENS is $410.64 \mathrm{MW}$.

Table 3.2 Total Costs and Execution Times of Two Methods for the 118-Bus System

\begin{tabular}{|c|c|c|c|c|l|}
\hline Method & $\begin{array}{c}\text { Cost } \\
(\$ / \text { hour })\end{array}$ & $\begin{array}{c}\text { EENS } \\
(\text { MWh })\end{array}$ & $\begin{array}{c}\text { Scenarios [Min cost] - } \\
\text { Enumeration } \\
\text { LPMOVEs - TAD }\end{array}$ & $\begin{array}{c}\text { Execution } \\
\text { time } \\
(\text { Secs })\end{array}$ & Reserve \\
(MW) \\
\hline Enumeration & $49,162.87$ & 800.98 & $1,000[994]$ & $51,908.07$ & 891.23 \\
\hline TAD & $49,156.92$ & 809.65 & 9 & 6.55 & 896.43 \\
\hline
\end{tabular}

Note: Reserve requirement without EENS is 410.64 MW (3.10)

Figure 3.7 gives the real power LMPs at all buses. The dashed line represents LMPs computed by the proposed OPF method with the TAD model of WEGs and the solid line represents LMPs calculated using the enumeration technique at the $994^{\text {th }}$ scenario. Once again, the solutions they have produced are a very close match, which demonstrates the accuracy of the proposed method. 


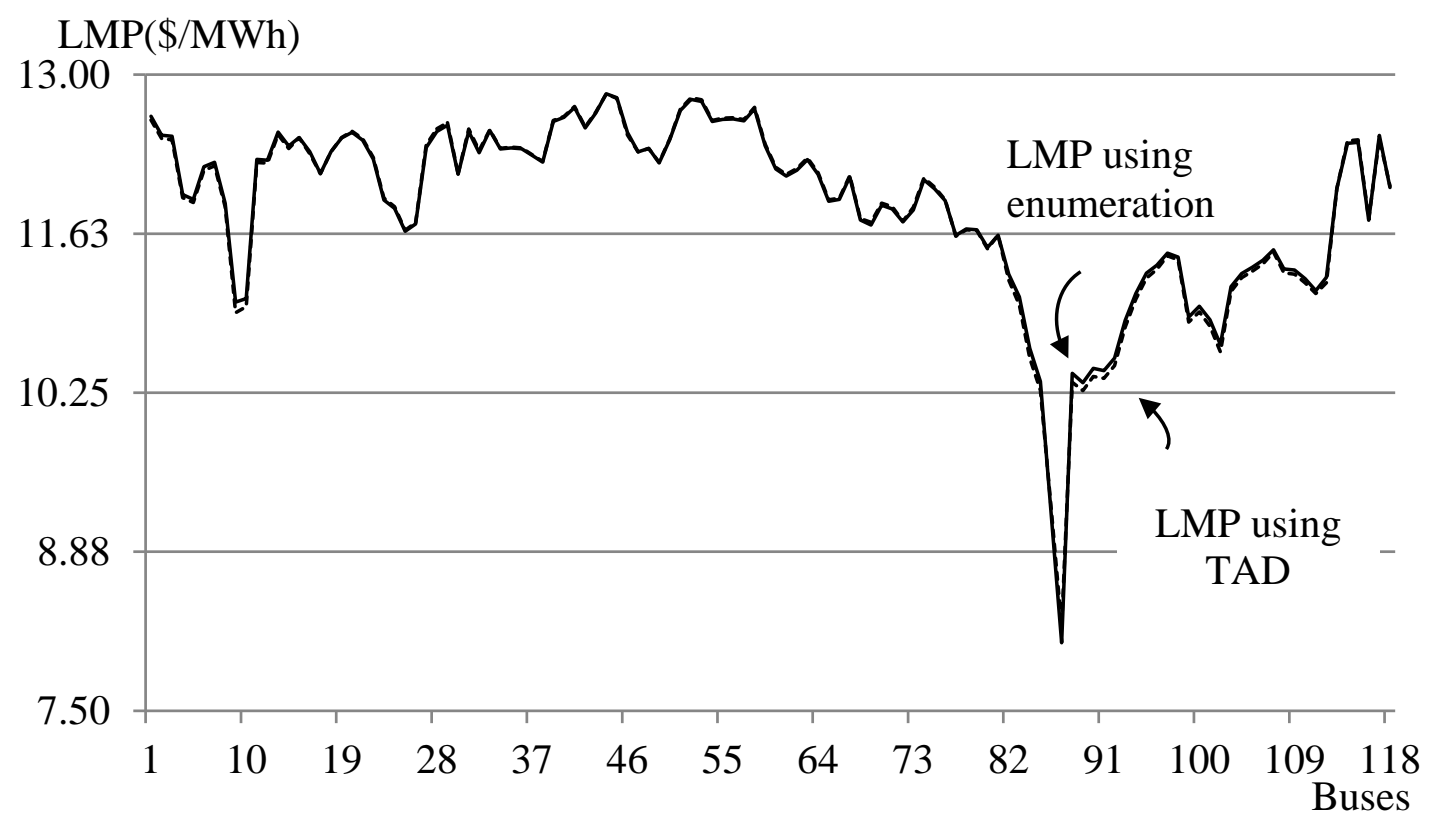

Fig. 3.7 Comparison of real power LMPs at all buses of 118-bus system

The IEEE 118-bus system was optimized using the fast OPF method with the proposed TAD model of WEGs for five different values of $\alpha$, the fraction of EENS value to be purchased as reserves, to see the effect on reserve cost and the total operating cost. The effects on the total operating cost and the output of WEGs are shown in Figure 3.8. The effects on the total reserve cost are shown in Figure 3.9. These graphs demonstrate that the proposed fast OPF method with TAD model schedules less power from WEGs to reduce the risk from EENS and corresponding reserve cost. 


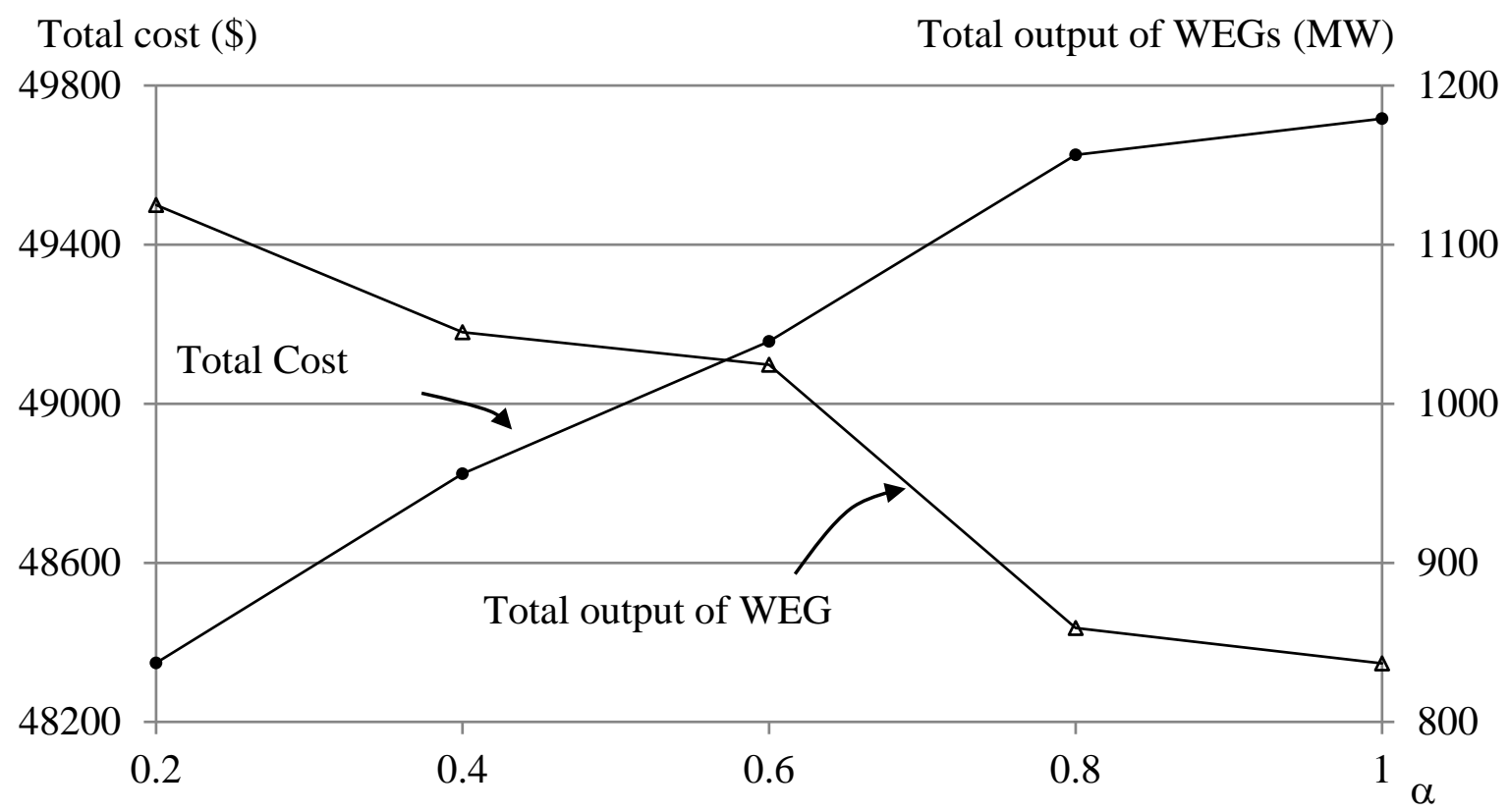

Fig. 3.8 Total cost and WEGs' output with different $\alpha$ values of the 118-bus system

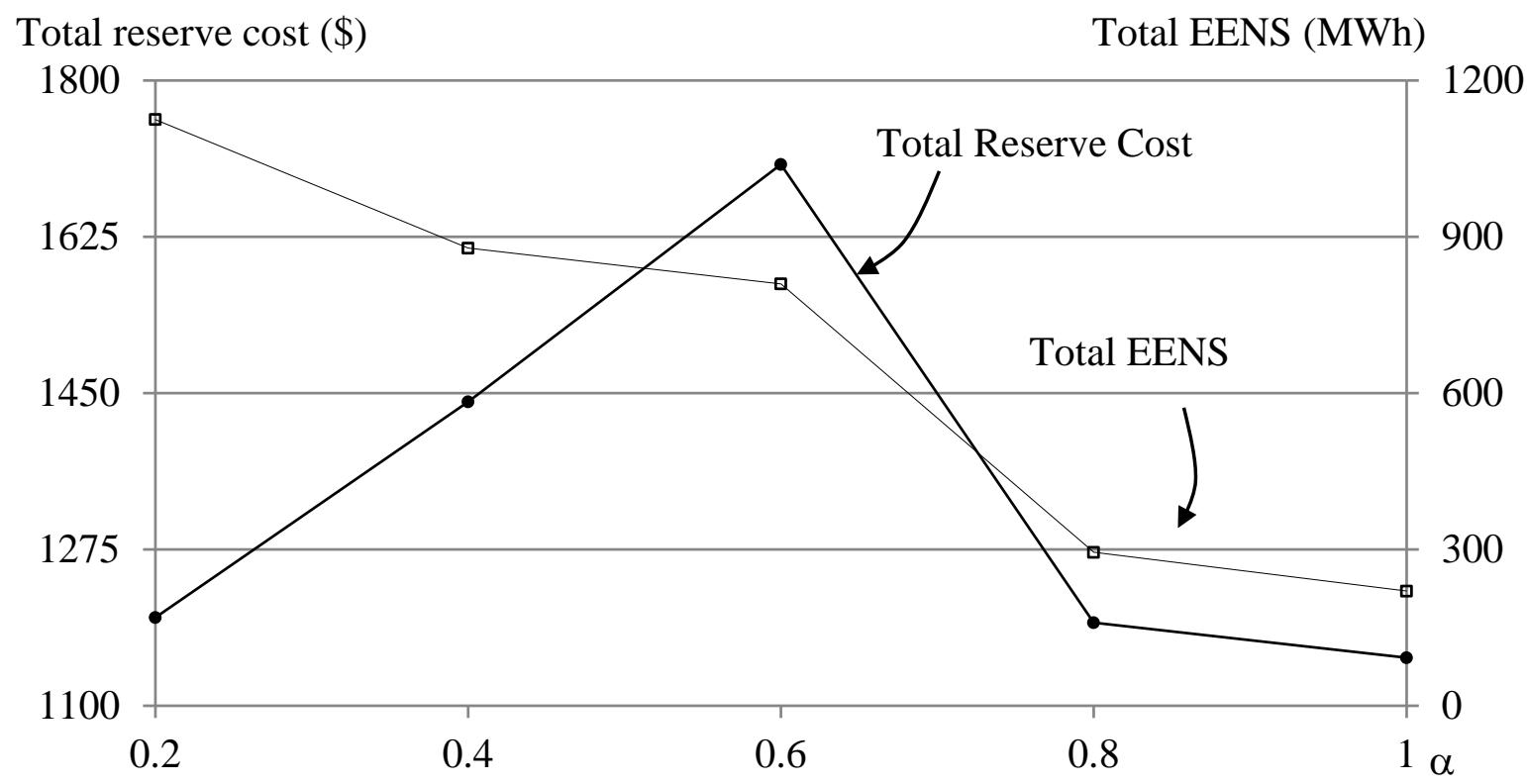

Fig. 3.9 Reserve cost and EENS with different $\alpha$ values of the 118-bus system 
Similar to the case of 30-bus system. With increasing of $\alpha$ values, the reserve cost increases to a peak and then decreases, as shown in figure 3.9. Considering the execution time, it is clear that the proposed fast OPF algorithm with the TAD model exhibits superior performance as compared to the enumeration approach. The proposed fast OPF algorithm with the TAD model can clearly save a great deal of computational time, which becomes more apparent for larger systems (Table 3.2).

This advantage in computational time highlights the practical fast applicability of the proposed fast OPF method with TAD model. The effect of varying standard deviation for both systems is discussed in next section.

\subsubsection{Effect of wind variability}

As discussed in Chapter 2, the value of standard deviation affects the working status of WEGs in the system operation (see Figures 2.4 and 2.5). For the same amount of power output from a WEG, a larger standard deviation value has a lesser EENS value when the WEG power output is more than the mean forecast. Furthermore, a lower value of EENS requires lesser spinning reserve to safeguard the system. This allows the system to consume more power from the WEG if it has a larger standard deviation in its generation output. Therefore, the total optimal cost decreases progressively with a larger standard deviation.

Table 3.3 shows the results of the 30 -bus and 118-bus systems with varying standard

deviation values from $5 \%$ to $15 \%$ of mean value ( $(\overline{\overline{P G}})$. In (3.10) and (3.26), the value of $\alpha$ is kept constant at 0.6. In these two systems, the total optimal costs decreases when standard deviation increases. 
Table 3.3 Total cost with different $\sigma$ for 30 and 118-bus systems

\begin{tabular}{|c|c|c|c|c|}
\hline \multirow{3}{*}{30 -bus } & $\sigma(=\% \overline{\overline{P G}})$ & $5 \%$ & $10 \%$ & $15 \%$ \\
\cline { 2 - 5 } & WEG(MW) & 105.01 & 108.67 & 115.08 \\
\hline \multirow{2}{*}{ 118-bus } & Total cost (\$) & $2,945.26$ & $2,935.84$ & $2,927.05$ \\
\cline { 2 - 5 } & WEG(MW) & 963.17 & 1024.51 & 1081.02 \\
\cline { 2 - 5 } & Total cost (\$) & $49,378.88$ & $49,156.92$ & $48,970.52$ \\
\hline
\end{tabular}

Using the proposed fast OPF algorithm with the TAD model of WEGs, one can easily analyze a system with various standard deviation values and obtain the optimal solution. Without the proposed tool, performing such an analysis is very cumbersome and extremely time consuming. This demonstrates the value and benefit of the proposed fast OPF algorithm with the TAD model of WEGs.

\subsection{Chapter Summary}

The TAD model of WEGs and the EENS function are incorporated into a fast OPF algorithm, which minimizes the total schedule costs including the cost of offsetting EENS by purchasing reserves to counter uncertainty. The proposed method uses the SLP technique to determine the optimal solution.

The proposed fast OPF method with the TAD model of WEGs is used to schedule modified IEEE 30-bus and 118-bus systems. Results from these studies are compared with the classical technique of enumeration. The comparison shows that the two methods produce very close 
results. However, the execution time for the proposed method is in the order of a few seconds while the enumeration technique requires computational time in the order of $10^{3}$ to $10^{5}$ seconds for a system with three WEGs. While the proposed technique remains stable in terms of time requirements for a large number of WEGs, the enumeration technique is unreasonable for use in real time, as its execution time increases exponentially.

These results clearly demonstrate the benefits of the proposed method: its accuracy in results and execution time advantage over enumeration technique. The benefits make the proposed OPF method suitable for practical applications.

As a next step, the benefits of using the TAD model of WEGs for large dimensioned problems such as the SCUC problem are explored in later chapters. In order to build a risk constrained SCUC method using TAD model of WEGs, it is necessarily to model full AC transmission security constraints as a first step. A successive fuzzy MILP method for SCUC with AC transmission constraints is discussed in the next chapter. 


\section{ChAPTER 4}

\section{SUCCESSIVE FUZZY MILP METHOD FOR AC}

\section{SECURITY CONSTRAINED UNIT COMMITMENT}

Modern power systems are facing a major change in their generation portfolio by connecting increasing amounts of renewable sources. One example is the province of Ontario, Canada, which operates according to the Green Energy Act [61]. This Act, through directive, has forced this power system to change its process for operation; formerly designed to work with fossil and nuclear fuel plants, this power system must now adapt to incorporate new renewable energy sources, which causes it to function in challenging operational states. Thus, it is highly desirable for the day-ahead security constrained unit commitment (SCUC) formulation to consider 24 sets of AC power balance equations - one for each hour - in a 24-hour scheduling period.

The SCUC algorithm is an essential tool for independent system operators (ISO) in their daily operations. It must have the ability to schedule generation during transmission network contingencies and to plan for short-term transmission maintenance outages. In this work, the proposed SCUC algorithm provides a near-optimal generator schedule for short-term 
electricity market, enforces transmission security constraints by fuzzy sets and minimizes transmission system MVA limit violations resulting from contingency and planned outage conditions.

The challenge of the fast OPF problem with WEGs was discussed in Chapter 3. The SCUC formulation considers 24 sets of those OPF formulations (one for each hour), considers ON/OFF status of generators and inter-temporal constraints such as ramp rate limits and up/down time limits. Thus, the SCUC is a much more complicated problem than the fast OPF because of its mixed integer feature, inter-temporal constraints and much high dimensionality.

In this chapter, a successive fuzzy MILP technique is proposed to tackle the SCUC problem with AC security constraints (AC-SCUC). It successively solves a fuzzy MILP formulation while updating integer and continuous variables. Several sizes of systems with severe transmission outages are examined. The details of the proposed fuzzy MILP method are discussed in the following sections.

\subsection{Fuzzy MILP Method for AC-SCUC}

Taking advantage of the fuzzy optimization technique, the AC-SCUC problem with violated security constraints can be solved in an infeasible region caused by transmission contingency or short-term planned transmission element outage. A fuzzy MILP formulation is solved successively while updating integer and continuous variables. The proposed fuzzy MILP formulation and approach is a variant of the SLP technique. The SLP technique is very efficient, it has been used to solve OPF formulations for several decades [62], and it has been 
successfully used to solve MILP formulations [63][64]. Several variants of the SLP have been investigated in the literature [65].

In addition to all other constraints of a conventional UC formulation, AC transmission system model and related constraints are enforced in the formulation reported in [44]. However, such AC transmission constraints are not directly formulated into UC algorithm by decomposition methods reported in literature. The AC transmission network-related constraints include: 1) real and reactive power balance equations at all the buses for all the hours, 2) line flow MVA limits, 3) limits on bus voltages, and 4) limits on reactive power outputs of generators.

The proposed AC-SCUC formulation poses a very large mixed integer nonlinear optimization challenge, and determining its solution using commercial solvers is very difficult. To address this challenge, this AC-SCUC formulation is linearized into an incremental MILP model. Further, fuzzy set theory is used to model infeasible constraints to allow the method to determine an optimal solution.

The process of formulating the MILP with AC transmission system constraints is a large and complex problem. Finding a feasible starting point may not be always easy, especially for the cases with severe transmission outages. To overcome this difficulty and to allow the proposed algorithm to start from an infeasible operating state, a special computing method is used to transform MILP formulation into a fuzzy MILP formulation. This fuzzy MILP model is solved using the MILP technique to minimize violations of constraints [66] while optimizing real power generation. The algorithm iterates successively until all constraint violations are removed (or minimized in cases with no feasible solution) and the optimal state is reached for 
a short-term (24-hour) generation schedule. The use of the MILP engine to solve the fuzzy MILP model in successive iterations makes the algorithm very reliable and computationally efficient. The details of this formulation are provided in the following sections.

\subsection{Deterministic formulation of AC-SCUC}

As discussed in Chapter 1, the day-ahead SCUC challenge is to schedule all the generators in the system for 24 hours before the dispatching day to minimize the total operation costs. The solution must satisfy constraints that limit the operation of generators and the connecting transmission network.

In order to isolate the characteristics of the AC transmission security constraints and the effects caused by transmission outages, WEGs are not considered in this chapter but will be dealt with in the next chapter - using the TAD model of WEGs proposed in Chapter 2.

The objective function of AC-SCUC is given in (4.1):

Minimize: TC $=\sum_{t} \sum_{n \in C C L}\left[a c_{n} \cdot U_{t n}+\sum_{m}\left(b c_{n m} \cdot P M_{t m m}\right)+r c_{n} \cdot S R_{t n}+S c_{n} \cdot S U_{t n}\right]$

Two sets of variables, $U_{t n}$ and $S U_{t n}$, are enforced as integer values of either 0 or 1 . They represent operational status and start-up status for the $n^{\text {th }}$ generator on the $t^{\text {th }}$ hour. Fixed costs $\left(a_{n}\right)$ for generators are borne by the system when they are turned on. Energy outputs of generators are segmented with each segment having its own price $\left(b c_{n m}\right)$. The cost of spinning reserve is obtained by multiplying reserve prices $\left(r c_{n}\right)$ by the corresponding scheduled capacity reserves. The price of start-up status $\left(s C_{n}\right)$ is also included in the objective function. 
By considering variations of time and linear segments of energy production, the UC formulation is a three-dimensional problem. The objective is subject to hourly and intertemporal constraints.

\section{1) Hourly constraints}

\section{Power balance equations:}

Real power: $\quad \sum_{n \in G I i} \sum_{m} P M_{t n m}-P D_{t i}=P T_{t i}\left(V_{t}, \delta_{t}\right) \forall t, i$

Reactive power: $\quad \sum_{n \in G I i} Q G_{t n}-Q D_{t i}=Q T_{t i}\left(V_{t}, \delta_{t}\right) \forall t, i$

In power balance equations (4.2) and (4.3), $P T_{t i}\left(V_{t}, \delta_{t}\right)$ and $Q T_{t i}\left(V_{t}, \delta_{t}\right)$ are the power flowing into the connected transmission system from the $i^{\text {th }}$ bus in the $t^{\text {th }}$ hour. These are functions of bus voltage phase angles and the magnitudes of all buses connected by transmission lines to the $i^{\text {th }}$ bus in the $t^{\text {th }}$ hour, as detailed in Appendix A. These power balance equations ensure that sufficient real and reactive power is scheduled to supply bus-wise demand and transmission losses. In addition to the OPF formulation discussed in the last Chapter, integer variables are used this AC-SCUC formulation to account for generator status.

Generator status of CEGs (integer variables): $\quad 0 \leq U_{t n} \leq 1 \quad \forall t, n \in C G$

$\underline{\text { Start-up status of CEGs (integer variables): }} S U_{t n}=\max \left\{U_{t n}-U_{t-1, n}, 0\right\} \forall t, n \in C G$

Additional constraints are added for variables $U_{t n}$ and $S U_{t n}$ to enforce integer values with values of 0 or 1 . A commercial software package MOSEK ${ }^{\circledR}$ is used to handle integer variables 
for this work [6]. All continuous variables, such as real power generation, are bounded within their respective limits.

Generator real power output limit:

Power limit in segments: $\quad 0 \leq P M_{n m} \leq \overline{P M_{n m}} \forall n \in C G, m$

$$
P G_{n}=\sum_{m} P M_{n m} \forall n \in C G
$$

Real power generation outputs are segmented into ' $M$ ' portions for the MILP formulation and are limited by respective segment bounds.

Power limits of all generator units:

$U_{t n} \cdot \underline{P G_{n}} \leq\left[P G_{t n}=\sum_{m} P M_{t n m}\right] \leq U_{t n} \cdot \overline{P G_{n}} \quad \forall t, n \in C G$

$\underline{P G_{n}} \leq P G_{t n} \leq \overline{P G_{n}} \quad \forall t, n \in W G$

System reserve constraints:

Minimum spinning reserve: $\quad \sum_{n \in C G} S R_{t n} \geq \gamma \cdot M R_{t} \quad \forall t$

Spinning reserve capacity:

$S R_{t n} \leq \min \left\{R 10_{n}, \overline{P G_{n}} \cdot U_{t n}-P G_{t n}\right\} \forall t, n \in C G$ 
System reserve criteria:

$$
\sum_{n \in C G} S R_{t n}+\sum_{n \in G 10}\left(1-U_{t n}\right) \cdot P 10_{n} \geq M R_{t} \forall t
$$

The factor $\gamma$ in (4.10) is the fraction of total hourly reserve to be scheduled by spinning reserve [59]. In this formulation, the reserve requirement is determined by the capacity of the largest online units. Quick start units (P10), which are offline and can be available in 10 minutes, are considered as additional reserve resources in (4.12).

Transmission networks are secured by AC security constraints, which include hourly line flow MVA limit, hourly reactive power generation limits, and hourly bus voltage constraints.

Transmission line flows: $\quad 0 \leq S F_{t l}\left(V_{t}, \delta_{t}\right) \leq \overline{S F_{l}} \forall t, l$

Reactive power outputs of generators: $\quad U_{t n} \cdot \underline{Q G_{n}} \leq Q G_{t n} \leq U_{t n} \cdot \overline{Q G_{n}} \forall t, n$

Voltage limits:

Voltage magnitude: $\quad \underline{V_{i}} \leq V_{t i} \leq \bar{V}_{i} \quad \forall t, i$

Voltage phase angles: $\quad-\pi \leq \delta_{t i} \leq \pi \quad \forall t, i$

In addition to the above hourly constraints, the SCUC problem is also restricted by intertemporal constraints over a 24-hour time horizon, as given below. 


\section{2) Inter-temporal constraints}

Ramp Rate: $\quad-R 60_{n} \leq P G_{t n}-P G_{t-1, n} \leq R 60_{n} \quad \forall t, n \in C G$

The ramp rate constraint restricts the capability of CEGs to raise or reduce generation for the next hour.

Minimum up/down time constraints:

Up time:

$\left(U_{t+1, n}-U_{t n}\right) \cdot U T_{n}-\sum_{s=t+2}^{\min \left\{N H, t+U T_{n}\right\}} U_{s n} \leq \max \left\{1, U T_{n}-N H+t+1\right\} \forall t \in[1, N H-2], n \in C G$

Down time: $\left(U_{t n}-U_{t+1, n}\right) \cdot D T_{n}+\sum_{s=t+2}^{\min \left\{N H, t+D T_{n}\right\}} U_{s n} \leq D T_{n} \quad \forall t \in[1, N H-2], n \in C G$

Initial condition constraints:

Up time: If I $C_{n}>0 \& U T_{n}>+I C_{n}$; then $U_{t n}=1 \forall t \in\left[1, U T_{n}-I C_{n}\right], n \in C G$

Down time: If $I C_{n}<0 \& D T_{n}>-I C_{n}$; then $U_{t n}=0 \forall t \in\left[1, D T_{n}+I C_{n}\right], n \in C G$

Due to economical and mechanical considerations on CEGs, these units are limited by minimum up/down constraints. A CEG must remain online (or offline) for a specific minimum time period, as it is not economically and mechanically safe to switch large generators on and off intermittently. Such intermittency is particularly dangerous for large nuclear generators. 
In the above formulation, the set of power balance equations and transmission line flows of the transmission network are nonlinear equations. Hence, the problem poses a mixed integer nonlinear programming (MINP) challenge.

This AC-SCUC challenge with AC transmission security constraints is dimensionally challenging even for small systems, and very hard to solve using any commercial mixed integer nonlinear programming solver. Alternatively, this research proposes to solve the AC-SCUC challenge by linearizing the MINP formulation and successively solving it using a robust mixed integer linear programming (MILP) solver. This linearized formulation of AC-SCUC is provided in the next section.

\subsection{Linearization (MILP formulation) of AC-SCUC}

The AC-SCUC problem has a control vector $X=[P G, U, S U, S R, V G]$, including real power generation, generator status, start-up status, system reserves, and voltage magnitudes on generator buses. This problem also has a dependent Vector $Y=[\delta, V L, Q G, S F]$, consisting of voltage angles on all buses, voltage magnitudes on load buses, reactive power output of generators, and MVA line flow. The NMIP optimization problem is linearized to formulate an incremental MILP problem using the current state $\left(X^{0}, Y^{0}\right)$. The objective function of the MILP AC-SCUC is to minimize change in the total operating costs $(\Delta \mathrm{TC})$ in terms of the incremental variables: $\Delta X=[\Delta P G, \Delta U, \Delta S U, \Delta R, \Delta V G]$ and $\Delta Y=[\Delta \delta, \Delta V L, \Delta Q G, \Delta S F]$ :

Objective: Minimize $\Delta \mathrm{TC}$ :

$$
\sum_{t} \sum_{n \in C G}\left(a c_{n} \cdot \Delta U_{t n}+\left(\sum_{m} b c_{n m} \cdot \Delta P M_{t n m}\right)+r c_{n} \cdot \Delta R_{t n}+s c_{n} \cdot \Delta S U_{t n}\right)
$$


This objective is subject to the following linearized equality and inequality constraints:

\section{Linear AC power balance equations:}

Generator buses (real and reactive power): $\sum_{n \in G l i} \sum_{m} \Delta P M_{t n m}=\Delta P T_{t i}\left(\Delta \delta_{t}, \Delta V_{t}\right) \forall t, i$

$$
\sum_{n \in G I i} \Delta Q G_{t n}=\Delta Q T_{t i}\left(\Delta \delta_{t}, \Delta V_{t}\right) \forall t, i
$$

Load buses (real and reactive power): $\quad 0=\Delta P T_{t i}\left(\Delta \delta_{t}, \Delta V_{t}\right) \quad \forall t, i$

$$
0=\Delta Q T_{t i}\left(\Delta \delta_{t}, \Delta V_{t}\right) \quad \forall t, i
$$

Generator status:

$$
0 \leq U_{t n}^{0}+\Delta U_{t n} \leq 1 \quad \forall t, n \in C G
$$

$\underline{\text { Start-up variables: }}$

$S U_{t n}^{0}+\Delta S U_{t n}=\max \left\{\left(U_{t n}^{0}+\Delta U_{t n}\right)-\left(U_{t-1, n}^{0}+\Delta U_{t-1, n}\right), 0\right\} \forall t, n \in C G$

Generation limit: $\quad 0 \leq P M_{t n m}^{0}+\Delta P M_{t n m} \leq \overline{P M_{n m}} \quad \forall t, n \in C G, m$

$$
\Delta P G_{t n}=\sum_{m} \Delta P M_{t n m} \forall t, n \in C G
$$

$\left(U_{t n}^{0}+\Delta U_{t n}\right) \cdot \underline{P G_{n}} \leq\left(P G_{t n}^{0}+\Delta P G_{t n}\right) \leq\left(U_{t n}^{0}+\Delta U_{t n}\right) \cdot \overline{P G_{n}} \quad \forall t, n \in C G$

$\underline{P G_{n}} \leq\left(P G_{t n}^{0}+\Delta P G_{t n}\right) \leq \overline{P G_{n}} \quad \forall t, n \in W G$ 


\section{Spinning reserve constraints:}

$\left(S R_{t n}^{0}+\Delta S R_{t n}\right) \leq \min \left\{R 10_{n}, \overline{P G_{n}} \cdot\left(U_{t n}^{0}+\Delta U_{t n}\right)-\left(P G_{t n}^{0}+\Delta P G_{t n}\right)\right\} \forall t, n \in C G$

$\sum_{n}\left(S R_{t n}^{0}+\Delta S R_{t n}\right) \geq \gamma \cdot M R_{t} \quad \forall t$

$$
\sum_{n \in C G}\left(S R_{t n}^{0}+\Delta S R_{t n}\right)+\sum_{n \in G 10}\left(1-\left(U_{t n}^{0}+\Delta U_{t n}\right)\right) \cdot P 10_{n} \geq M R_{t} \forall t
$$

Linear MVA line flow constraints: $\quad 0 \leq S F_{t l}^{0}+\Delta S F_{t l}\left(\Delta \delta_{t}, \Delta V_{t}\right) \leq \overline{S F_{l}} \forall t, l$

\section{Linear reactive power limit:}

$\left(U_{t n}+\Delta U_{t n}\right) \cdot Q G_{n} \leq Q G_{t n}^{0}+\Delta Q G_{t n} \leq\left(U_{t n}+\Delta U_{t n}\right) \cdot \overline{Q G_{n}} \forall t, n$

Bus voltage limits:

Magnitude: $\quad V_{i} \leq V_{t i}^{0}+\Delta V_{t i} \leq \bar{V}_{i} \quad \forall t, i$

Phase angles: $\quad-\pi \leq \delta_{t i}^{0}+\Delta \delta_{t i} \leq \pi \quad \forall t, i$

Ramp rate: $\quad-R 60_{n} \leq\left(P G_{t n}^{0}+\Delta P G_{t n}\right)-\left(P G_{t-1, n}^{0}+\Delta P G_{t-1, n}\right) \leq R 60_{n} \quad \forall t, n \in C G$

Minimum up/down time constraints:

$\left[\left(U_{t+1, n}^{0}+\Delta U_{t+1, n}\right)-\left(U_{t n}^{0}+\Delta U_{t n}\right)\right] \cdot U T_{n}-\sum_{s=t+2}^{\min \left\{N H, t+U T_{n}\right\}}\left(U_{t n}^{0}+\Delta U_{s n}\right) \leq \max \left\{1, U T_{n}-N H+t+1\right\}$

$\forall t \in[1, N H-2], n \in C G$ 
$\left.\left[\left(U_{t+1, n}^{0}+\Delta U_{t+1, n}\right)-\left(U_{t n}^{0}+\Delta U_{t n}\right)\right]\right] D T_{n}+\sum_{s=t+2}^{\min \left\{N H, t+P_{\left.T_{n}\right\}}\right\}}\left(U_{s n}^{0}+\Delta U_{s n}\right) \leq D T_{n}$

$\forall t \in[1, N H-2], n \in C G$

Initial conditions: If I $C_{n}>0 \& U T_{n}>+I C_{n}$; then $\Delta U_{t n}=0 \forall t \in\left[1, U T_{n}-I C_{n}\right], n \in C G$

$$
\text { If } I C_{n}<0 \& D T_{n}>-I C_{n} ; \text { then } \Delta U_{t n}=0 \forall t \in\left[1, D T_{n}+I C_{n}\right], n \in C G
$$

Hourly and inter-temporal constraints are linearized around the current operating state. Reserve constraints are enforced to guarantee adequate generation capacity. In (4.24) - (4.26), the bus wise linear functions $\Delta P T_{t i}$ and $\Delta Q T_{t i}$ are formed using the system Jacobian, one for each hour. In matrix form, they are defined as follows, where $[J 1]_{t},[J 2]_{t},[J 3]_{t}$, and $[J 4]_{t}$ are the appropriate parts of the system Jacobian for the $\mathrm{t}^{\text {th }}$ hour:

$$
\begin{aligned}
& \Delta P T_{t}=[J 1]_{t} \cdot \Delta \delta_{t}+[J 2]_{t} \cdot \Delta V_{t} \quad \forall t \\
& \Delta Q T_{t}=[J 3]_{t} \cdot \Delta \delta_{t}+[J 4]_{t} \cdot \Delta V_{t} \quad \forall t
\end{aligned}
$$

The change in the MVA line flow, (4.36), can be expressed as a linear relation, as shown below:

$$
\Delta S F_{t l}=[J D L]_{t l} \cdot \Delta \delta_{t}+[J V L]_{t l} \cdot \Delta V_{t} \quad \forall t, l
$$

In (4.47), $[J D L]_{t l}$ and $[J V L]_{t l}$ are sensitivity matrices related to bus voltage magnitudes and phase angles, and they are evaluated at the current state. 
Additionally, constraints are added so that the summation of $\left(U_{t n}+\Delta U_{t n}\right)$ results in an integer value of 0 or 1 for generator status. Similarly, constraints are added so that the sum of $\left(S U_{t n}+\Delta S U_{t n}\right)$ results in an integer value of 0 or 1 for generator start-up.

This MILP model is solved to determine the best change to cost ( $\Delta \mathrm{TC})$ in order to minimize total operational cost $\left(\mathrm{TC}^{0}+\Delta \mathrm{TC}\right)$. The optimal change $(\Delta X)$ determined by the MILP are used to update the control vector $\left(X=X^{0}+\Delta X\right)$. The dependent vector $(Y)$ is updated by solving bus-wise power balance equations using Newton-Raphson technique, or using an OPF if violations exist on reactive power control variables. This process of formulating the MILP model, obtaining a solution of control variables, and updating the state of dependent variables by solving Power Flow/OPF constitutes an LPMOVE (Figure 4.6). This process is repeated until the optimal solution is reached.

In each LPMOVE, the MILP formulation and its solution produces the optimal changes in the continuous and integer variables, $\Delta X=[\Delta P G, \Delta U, \Delta S U, \Delta S R, \Delta V G]$ and $\Delta Y=[\Delta \delta, \Delta V L, \Delta Q G, \Delta S F]$, such that the change in the total operating cost (4.22) $\Delta \mathrm{TC}$ is minimized while all the operating and control constraints are satisfied.

This formulation (4.22) - (4.44) is large and numerically challenging, as it combines 24hourly solutions via inter-temporal constraints. Often, a small violation renders the entire solution infeasible. Furthermore, under conditions of contingency or planned transmission line outage, violations on transmission constraints are significant and unavoidable. In order to solve the problem from an infeasible state, a fuzzy optimization process is used to minimize operating costs (4.1) and simultaneously minimize constraint violations. Accordingly, the objective (4.22) and violated constraints are transformed into fuzzy models. Thereafter, the 
MILP formulation (4.22) - (4.44) is transformed into a fuzzy MILP formulation. The fuzzy model of violated constraints ensures that the solution remains feasible and violations are minimized. These fuzzy objective and constraint equations developed for the linear formulation are described in the following section.

\subsection{Fuzzy Constraints for AC-SCUC}

The linear formulation of AC-SCUC is transformed into a fuzzy MILP formulation by creating fuzzy models of the objective and violated constraints, while retaining other constraints intact.

Solving the power balance equations (by power flow or OPF) for 24 hours may show violations of line flow limits and voltage magnitude limits. Although it may not be possible to eliminate violations of all constraints in a single LPMOVE process, intelligent optimization methods such as the fuzzy optimization technique have been used to overcome infeasibility and to provide a pragmatic algorithm. The fuzzy sets theory is a very effective and widely used tool to handle engineering problems with conflicting drivers [6], [64]-[66]. The incremental cost function (4.21), line flow constraint (4.35), and bus voltage magnitude constraint (4.37) are transformed into fuzzy constraints, as shown below.

\subsubsection{Fuzzy set of objective function}

A fuzzy set of cost function (4.22) is defined by creating a set of values for $\Delta \mathrm{TC}$ such that: $\underline{\Delta T C} \leq \Delta T C \leq \overline{\Delta T C}$. The satisfaction for this fuzzy set is defined below (also refer to Figure 4.1): 
$\mu T C=\frac{\overline{\Delta T C}-\Delta T C(X)}{\overline{\Delta T C}-\underline{\Delta T C}}$

The values of $\underline{\Delta T C}$ and $\overline{\Delta T C}$ are computed by assessing the minimum and maximum values of control vectors ( $\Delta P G, \Delta U, \Delta S U, \Delta S R, \Delta V G)$ and using them in (4.22).

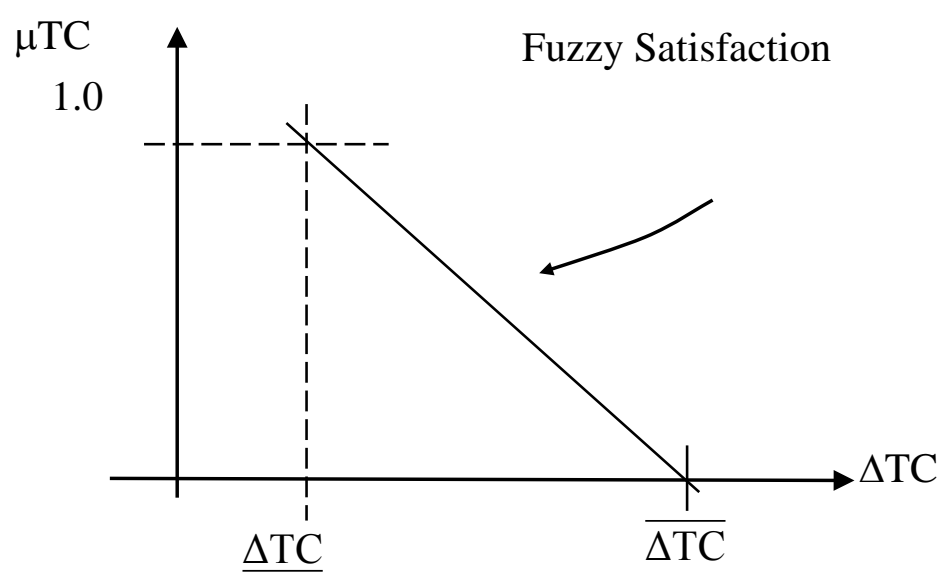

Fig. 4.1 Fuzzy set of Total Cost increment

\subsubsection{Fuzzy set of Line Flow Limit Constraint}

The MVA line flow is transformed to a fuzzy constraint when it violates constraint (4.36) in the current state $\left(S F_{t l}^{0}\right)$ of the $I^{\text {th }}$ line (two directions) in the $t^{\text {th }}$ hour. The value of line flow before optimization is $S F_{t l}^{0}$ and after optimization is $\left(S F_{t l}^{0}+\Delta S F_{t l}\right)$. With an upper limit 
violation, $\left(S F_{t l}^{0} \geq \overline{S F_{l}}\right.$ ), a fuzzy set is defined for the variable $\Delta S F_{t l}$ such that it satisfies $\overline{S F_{l}} \leq S F_{t l}^{0}+\Delta S F_{t l} \leq S F_{t l}^{0}$. The satisfaction function is defined as Figure 4.2:

$\mu S F_{t l}=\frac{\left(S F_{t l}^{0}\right)-\left(S F_{t l}^{0}+\Delta S F_{t l}\right)}{\left(S F_{t l}^{0}\right)-\overline{S F_{l}}}=\frac{-\Delta S F_{t l}}{\left(S F_{t l}^{0}\right)-\overline{S F_{l}}} \quad \forall t, l$

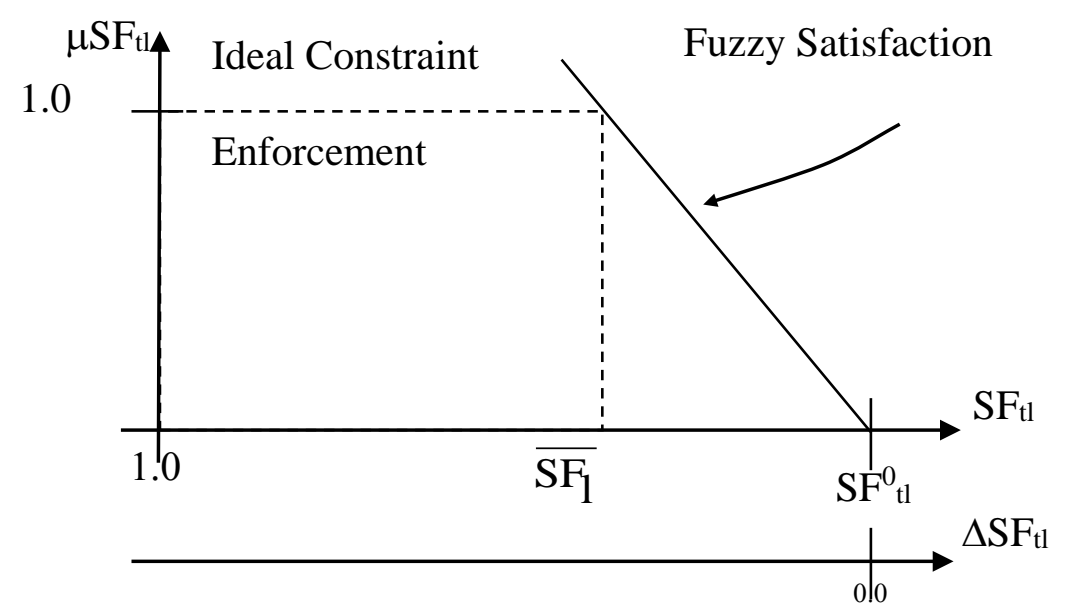

Fig. 4.2 Fuzzy set for Line Flow Limit Constraint

\subsubsection{Fuzzy sets of Voltage Limit Constraint}

When a load bus voltage magnitude in the current state $\left(V_{t i}^{0}\right)$ of the $i^{\text {th }}$ bus in the $t^{\text {th }}$ hour violates constraint (4.38), it is transformed into fuzzy constraints, as shown below. The value of voltage magnitude before optimization is $V_{t i}^{0}$ and after optimization is $V_{t i}^{0}+\Delta V_{t i}$. 
1) Upper Limit Violation: $\left(V_{t i}^{0} \geq \overline{V_{i}}\right)$

A fuzzy set is defined for the variable $\Delta V_{t i}$ such that it satisfies $\bar{V}_{i} \leq V_{t i}^{0}+\Delta V_{t i} \leq V_{t i}^{0}$. The satisfaction function is defined as Figure 4.3:

$\mu V L U_{t i}=\frac{\left(V_{t i}^{0}\right)-\left(V_{t i}^{0}+\Delta V_{t i}\right)}{\left(V_{t i}^{0}\right)-\bar{V}_{i}}=\frac{-\Delta V_{t i}}{\left(V_{t i}^{0}\right)-\bar{V}_{i}} \quad \forall t, i \in N L B$

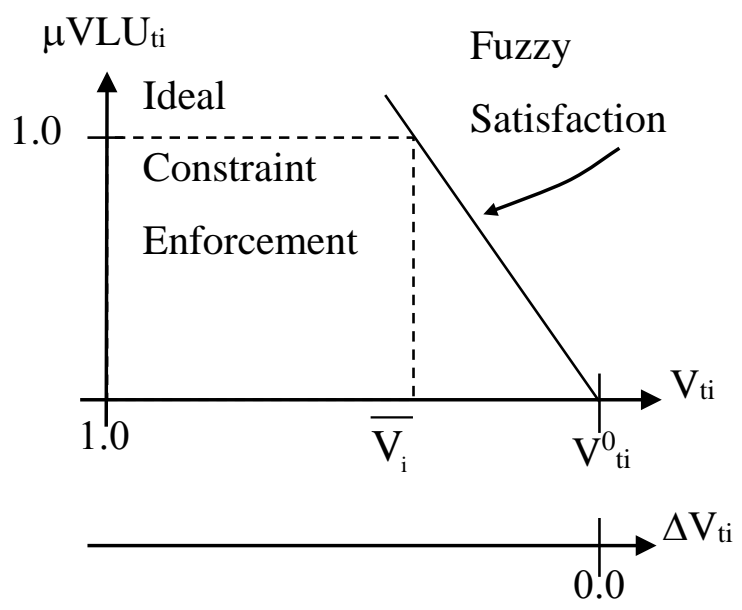

Fig. 4.3 Fuzzy set for Load Bus Voltage Upper Limit Constraint

2) Lower Limit Violation: $\left(V_{i} \leq V_{t i}^{0}\right)$

A fuzzy set is defined for the variable $\Delta V_{t i}$ such that it satisfies: $V_{t i}^{0} \leq V_{t i}^{0}+\Delta V_{t i} \leq \underline{V_{i}}$. The satisfaction function is defined as Figure 4.4: 


$$
\mu V L L_{t i}=\frac{\left(V_{t i}^{0}+\Delta V_{t i}\right)-\left(V_{t i}^{0}\right)}{\underline{V_{i}}-\left(V_{t i}^{0}\right)}=\frac{+\Delta V_{t i}}{\underline{V_{i}}-\left(V_{t i}^{0}\right)} \forall t, i \in N L B
$$

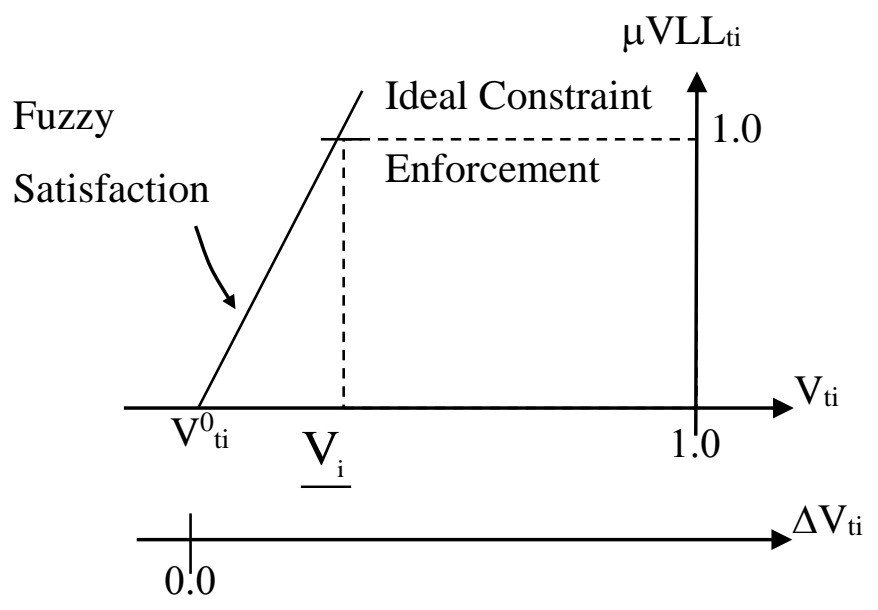

Fig. 4.4 Fuzzy set for Load Bus Voltage Lower Limit Constraint

\subsubsection{Fuzzy objective}

A variable $(\lambda)$ is defined as equal to the minimum of all satisfaction functions:

$\lambda=\operatorname{minimum}\{\mu T C, \mu S F, \mu V L U, \mu V L L\}$

The fuzzy MILP model is formulated as below. By maximizing fuzzy objective $\lambda$ the total cost is optimized, subject to the enforcement of constraints. Therefore, the fuzzy MILP is formulated as below:

Maximize: $\lambda$, 
Subject to $(4.23)-(4.44)$.

The above formulation is set up and solved to determine optimal values of $\Delta X$. The state $X$ is updated and the dependent vector $Y$ is determined using power flow/OPF. These steps constitute one LPMOVE step (see Figure 4.5). These LPMOVE steps are repeated until the optimal solution is reached and violations are removed. The complete algorithm is discussed in the next section.

\subsection{Algorithm of Successive Fuzzy MILP SCUC}

The algorithm of the proposed method is presented in Figure 4.5. As a first step in solving the proposed AC-SCUC challenge, a starting state is obtained by solving a UC formulation with DC network constraint (DC-SCUC) as well as a 24-set of power balance equations, such that the starting state has a set of 24 operating states.

The DC-SCUC is solved using an MILP technique by an efficient commercial optimization engine, and power balance equations are satisfied using Newton Raphson power flow method to obtain line flows, bus voltages and reactive power generation values for the period of 24 hours as the starting state of AC-SCUC. This starting state may not be optimal or network feasible or neither, especially under certain transmission line outages.

If the DC-SCUC cannot be solved for the original physical transmission line capacities with transmission contingency or line outage conditions, a virtual capacity is assumed (e.g., 1.5 times of capacity on the line) and used to obtain a feasible solution of DC-SCUC. The target of the proposed method is to minimize violations on transmission lines and to control such violations within a range of $20 \%$ overload when possible. 


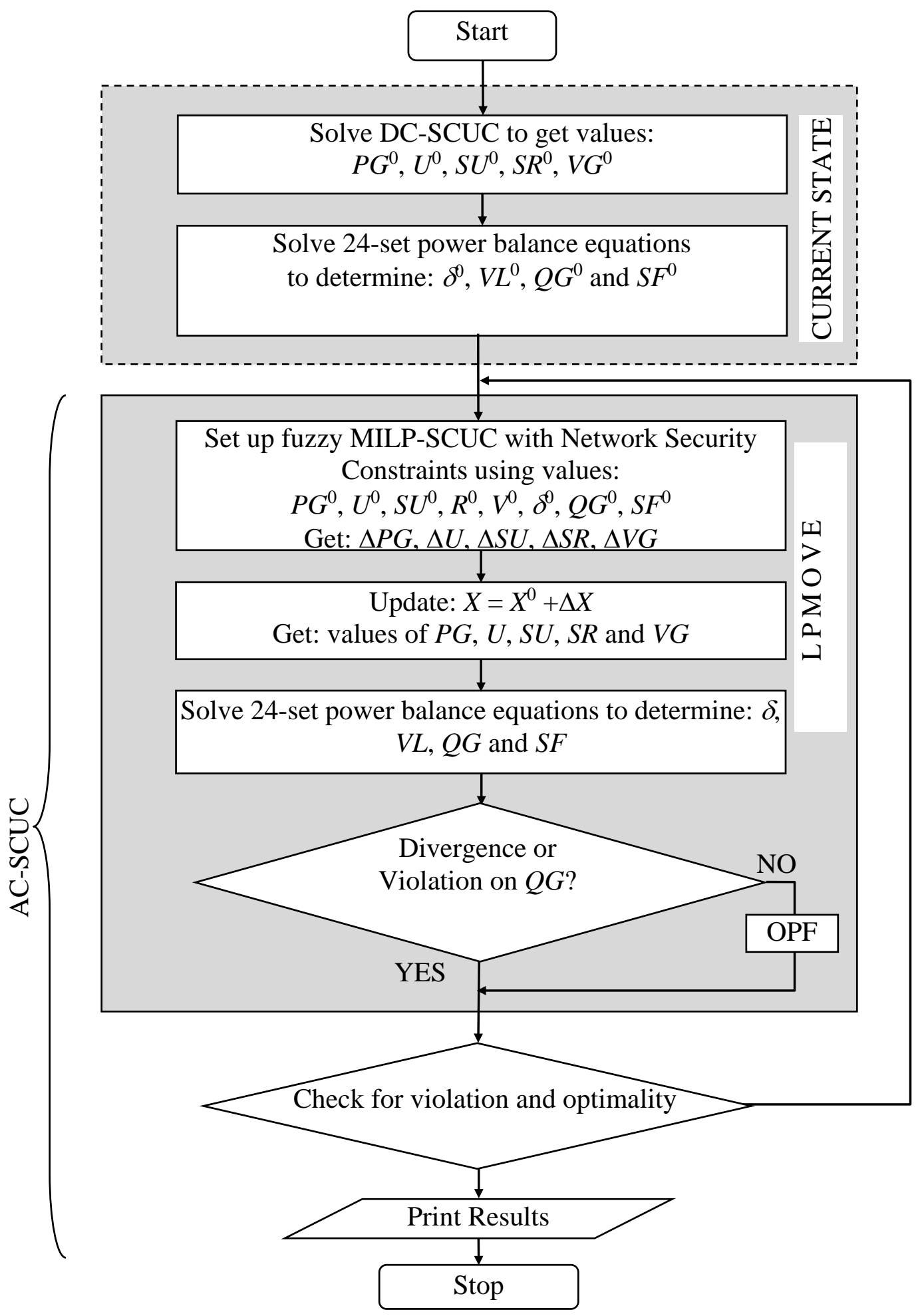

Fig. 4.5 Flowchart of the proposed AC-SCUC algorithm 
In the situations in which the power balance algorithm fails to converge in any hour, or reactive power generation violations exist, an additional OPF is solved. The detail of the OPF formulation used here is provided in Appendix A.

Thereafter, to minimize the total schedule cost while considering all constraints, a set of steps called LPMOVE is iteratively performed to reach the optimal solution. Each LPMOVE includes the steps of 1) formulating and solving the fuzzy MILP model, 2) updating variables using the optimal incremental values obtained from the fuzzy MILP solution, and 3) solving 24 sets of power balance equations with the updated state $\mathrm{X}$ using power flow or OPF. The program is terminated when the violation of each line flow is less than a small tolerance and total cost is minimized. The proposed successive fuzzy MILP formulation of AC-SCUC is tested on three systems with different transmission outage states. The details are described in next section.

\subsection{Test Results and Discussions}

In this section, the results of the tests are presented. Three systems are chosen as follows:

1) 6-bus transmission network with 7 transmission lines over a 3-hour time span.

2) A modified IEEE 57-bus transmission network with 76 transmission lines on a 24-hour time horizon.

3) The IEEE 118-bus transmission network with 186 transmission lines on a 24-hour time horizon. 
The six-bus system is chosen to illustrate features of the method and its merits, whereas the IEEE 57-bus and 118-bus systems are chosen to show how the method performs for medium and large sizes of systems with more complex transmission networks.

\subsubsection{6-bus system}

The 6-bus, 3-generator system has been chosen for a 3-hour time horizon. The generator cost data is altered such that Generator \#1 (CEG1) is the least expensive and Generator \#3 (CEG3) is the most expensive (see Appendix $\mathrm{C}$ for data). The 6-bus system is network capacity constrained. The Generator \#2 (CEG2) at bus \#2 is connected via five transmission lines to bus \#4. As bus \#2 has no load, the output of CEG2 must flow through the lines between buses \#2 and \#4 and is constrained by the capacity of these lines. In this study, the capacity of lines between buses \#2 and \#4 is limited to 210 MVA (42 MVA per line). Three different states are studied using the proposed successive fuzzy MILP formulation and are described in detail below.

\section{1) No transmission line outage}

All five lines are working properly in this state. Without transmission line outage, the total capacity of the transmission connection between bus \#2 and bus \#4 is 210 MVA. Thus, there is no violation on the lines. The proposed algorithm is working towards minimizing total operation cost. The starting state is obtained by solving DC-SCUC with assumed losses, 3\% of total demand. After solving the AC-SCUC problem, the actual loss (1.45\% of demand) is less than the assumed value, which reduces the cost. The convergence characteristic of this case is shown in Figure 4.6. Each LP represents one LPMOVE. 


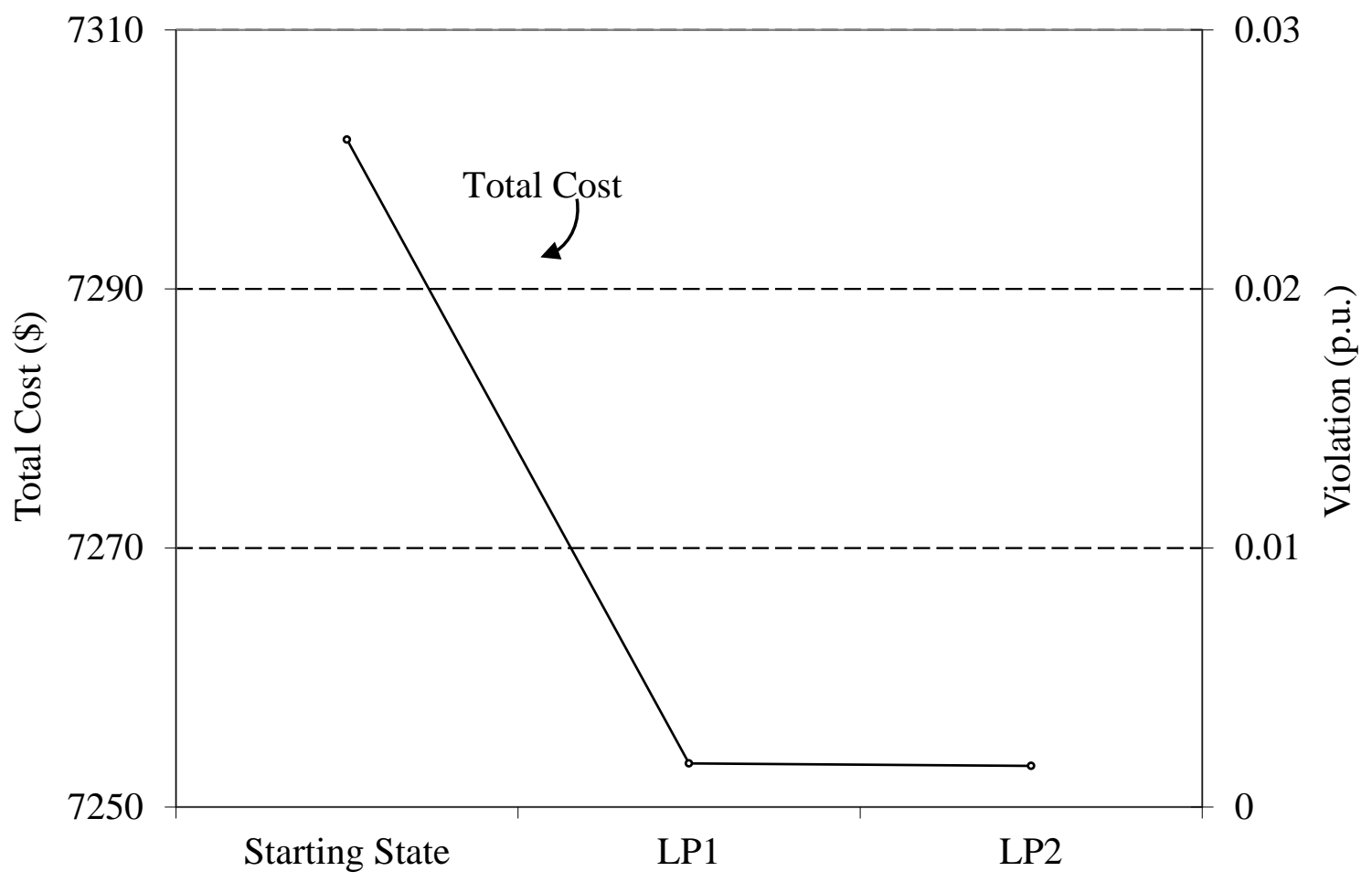

Fig. 4.6 Convergence characteristic of the 6-bus with no transmission outage

2) One transmission line is out of service

If transmission outage occurs among the lines between bus \#2 and bus \#4, the total network capacity is lower, and transmission violation will appear. One line outage is considered in this case. With one line out of service, the transmission capacity drops to 168 MVA.

The DC-SCUC is solved with transmission capacity of 168 MVA. After load flow and OPF, the starting state shows large violation on the lines. The proposed method minimizes transmission line violation and adjusts generation (see Figure 4.7). 


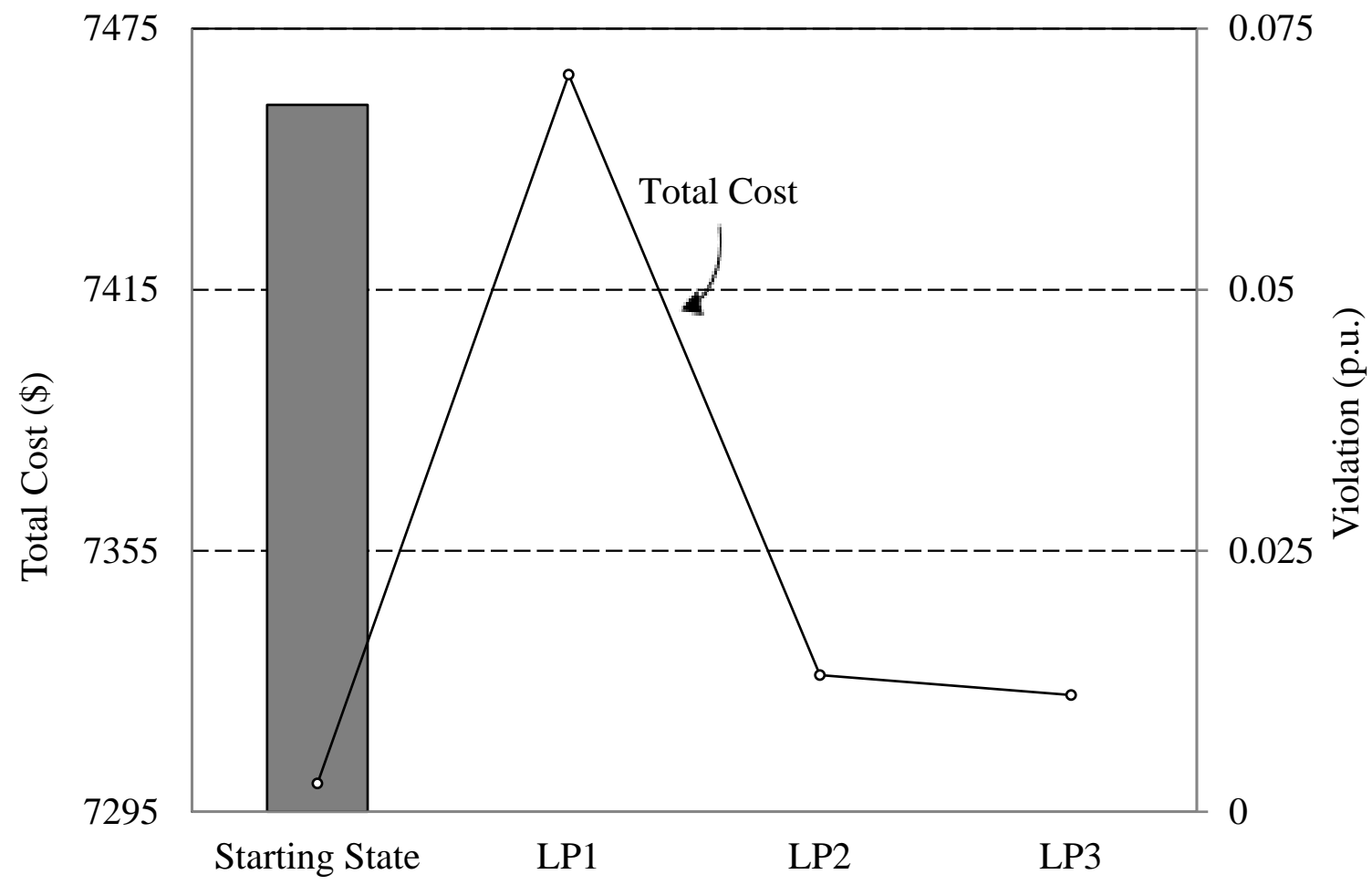

Fig. 4.7 Convergence characteristic of the 6-bus with one connection out

With one line out of service, transmission constraints are violated due to insufficient transmission capacity. A fuzzy set of MVA line flow limit is built (4.49). The fuzzy objective maximizes the least fuzzy membership satisfaction functions (4.52), which is the fuzzy set of transmission line MVA constraint. In this case, the total cost is increased during the process of removing transmission line violations and is reduced after the violations are cleared. 


\section{3) Two transmission lines are out}

In order to study the advantage of fuzzy constraints, one additional transmission line is planned for outage in this section. With two lines out of service, the remaining capacity of the transmission connection between bus \#2 and bus \#4 becomes 126 MVA ( 3 lines remaining). In this case, the DC-SCUC is unable to obtain a feasible solution. The starting state is obtained using DC-SCUC by increasing the line capacity to 1.5 times the rated value assuming that $50 \%$ overload is allowed for all lines to obtain a feasible DC-SCUC solution. After solving power flow and OPF, the transmission line violation is extremely large - 10 times larger than the second case with one line outage.

Figure 4.8 shows the convergence characteristics of the algorithm and that there is a line flow violation in the starting state, but this violation cannot be cleared. The advantage of the proposed algorithm is its ability to control limit violations within acceptable range of overload (20\%) for a short-term transmission emergency outage planning when possible and minimization of violations. This case study helps to validate the basic model, demonstrating the extent of its performance and capability. Details of this case are given in Table 4.1.

The final generation schedule and transmission line flows of the three cases are given in Table 4.2. The most expensive generator (CEG3) is turned on to reduce the generation of CEG2 and minimize line violations. 


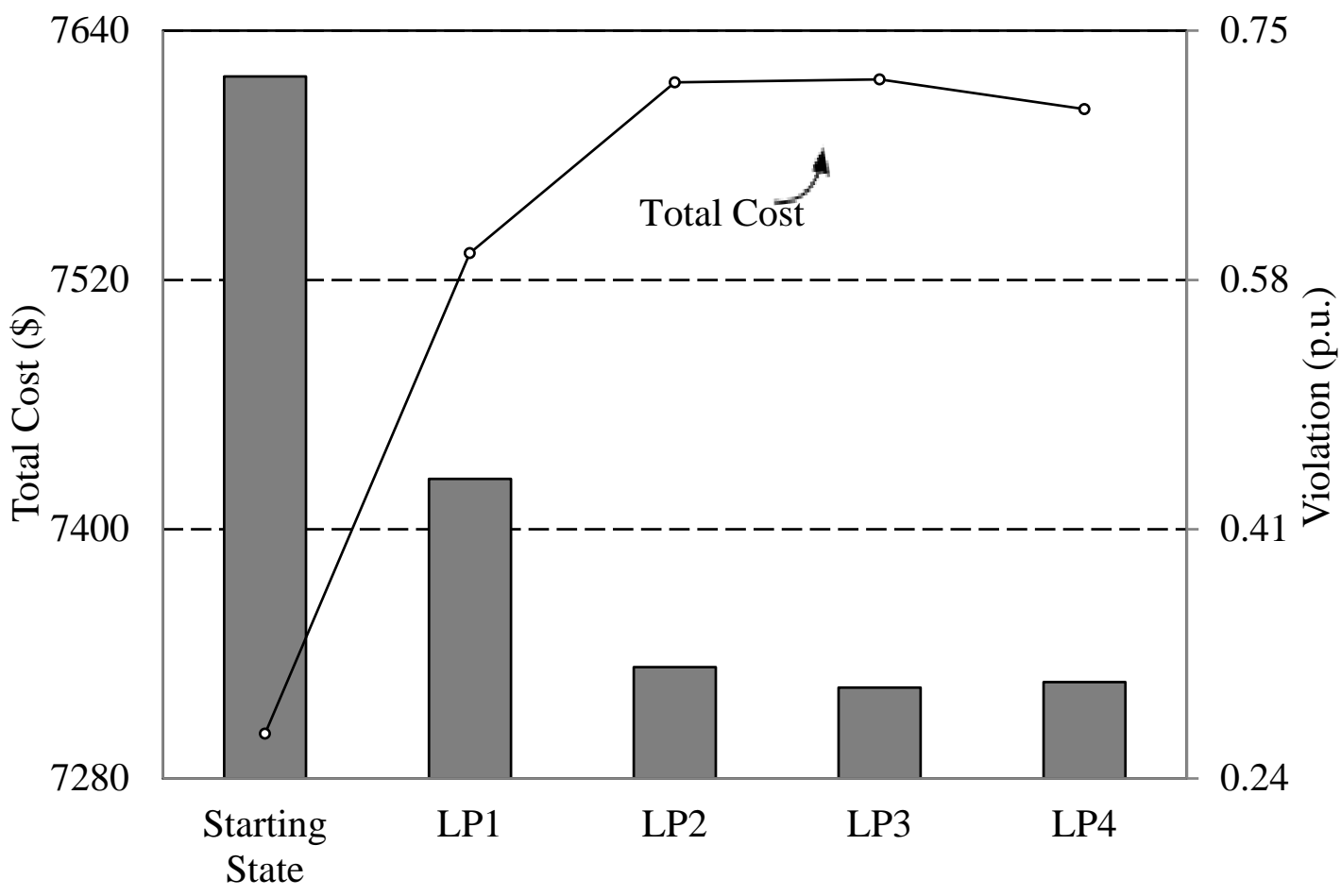

Fig. 4.8 Convergence characteristic of the 6-bus with two connections out

Table 4.1 Details of 6-bus System with transmission outage in 3 hours

\begin{tabular}{l|cll|c}
\hline System & 6-bus & & Integer Variables & 18 \\
\cline { 1 - 2 } \cline { 5 - 6 } Computer & Windows/64-X86 & & LPMOVEs & 4 \\
\cline { 1 - 2 } \cline { 5 - 6 } Constraints & 175 & & Total Schedule Cost (\$) & $7,602.26$ \\
\hline Variables & 100 & & Executing Time (Sec) & 3.32 \\
\hline
\end{tabular}


Table 4.2 Generation Schedules of 6-bus system in the final LPMOVE of 3 cases

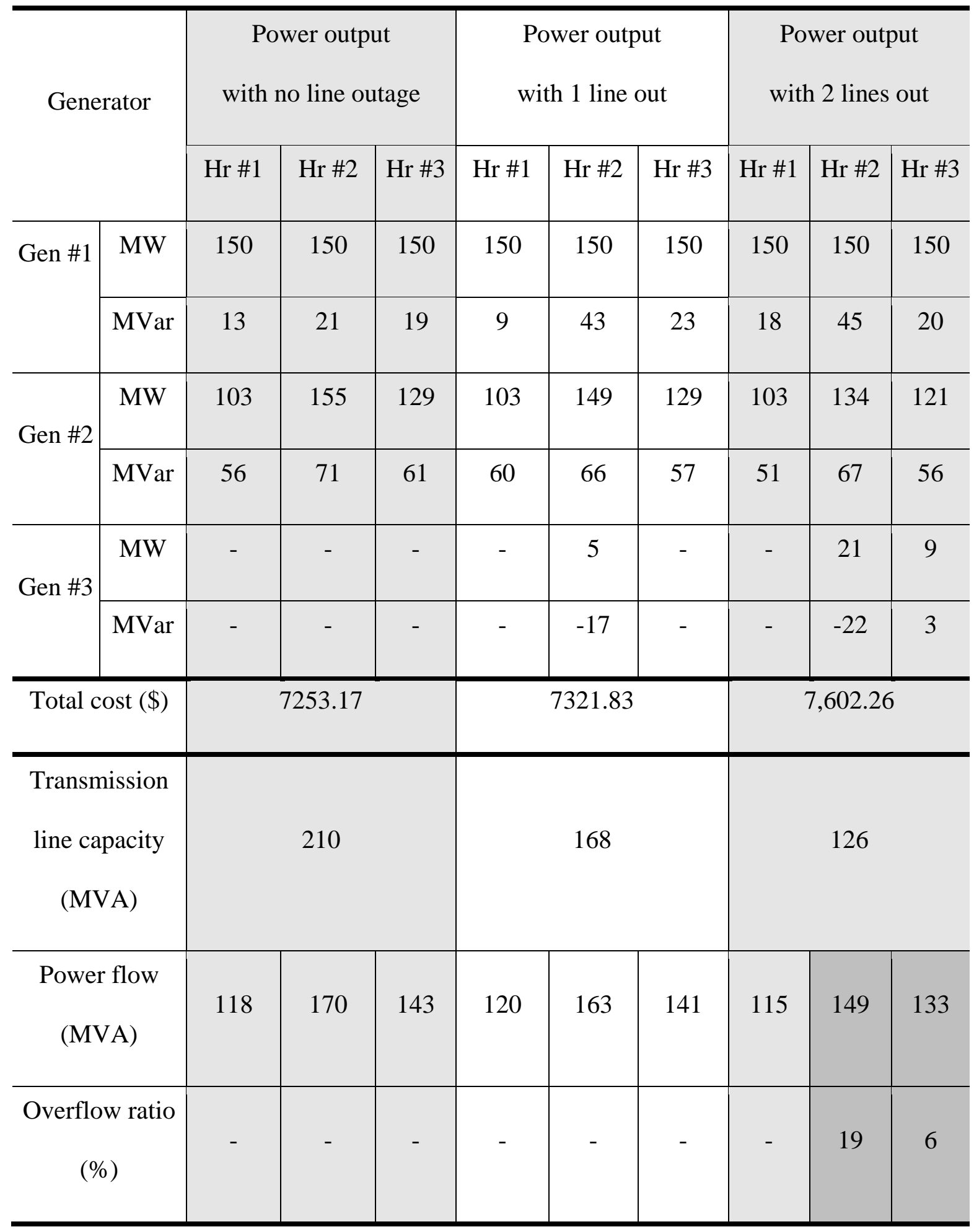




\subsubsection{IEEE 57-bus system}

The modified IEEE 57-bus power system has 26 generator buses, 31 load buses, and 76 transmission lines [6]. In order to show the effects of line outages and the advantage of the proposed fuzzy security constraints, the transmission line between bus \#8 and bus \#9 are altered into a multiple-line connection (3 lines). The capacity of each line is set as 100 MVA. Two different states are studied using the proposed successive fuzzy MILP method and are described in detail below.

\section{1) No transmission line outage}

By assuming all the lines are working properly, the total capacity is 300 MVA on the transmission connection between buses \#8 and \#9.

Similar to the tests for the 6-bus system, the DC-SCUC and AC power balance equations are solved for the starting state. With sufficient transmission line capacity, there is no violation. The proposed method minimizes total cost only, as shown in Figure 4.9.

\section{2) One transmission line is out of service}

Another case is studied with one line out of service. The network capacity between bus \#8 and bus \#9 drops to 200 MVA and violations appear in the remaining transmission lines between these two buses. Total cost and violations are shown in figure 4.10. 


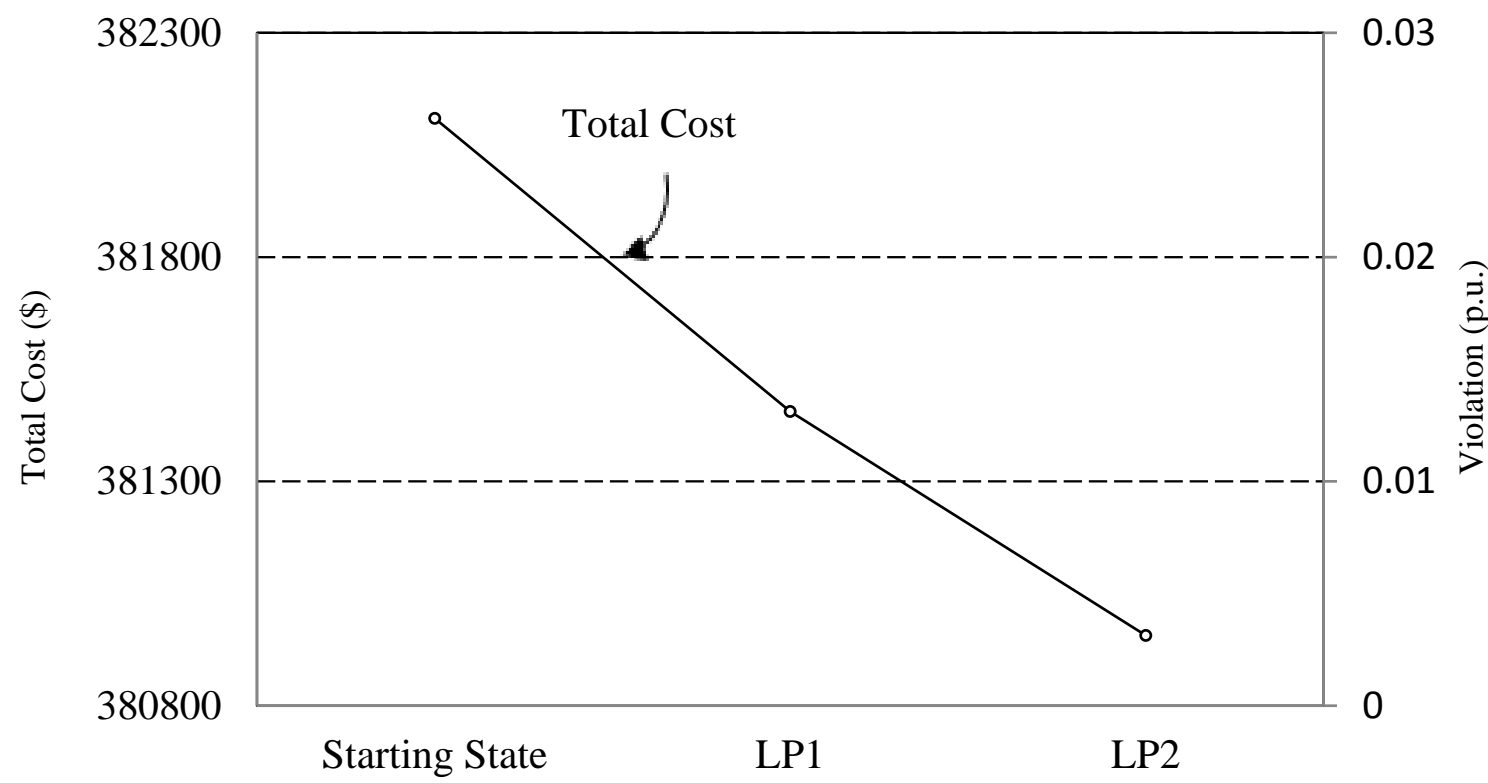

Fig. 4.9 Convergence characteristic of the 57-bus without outage

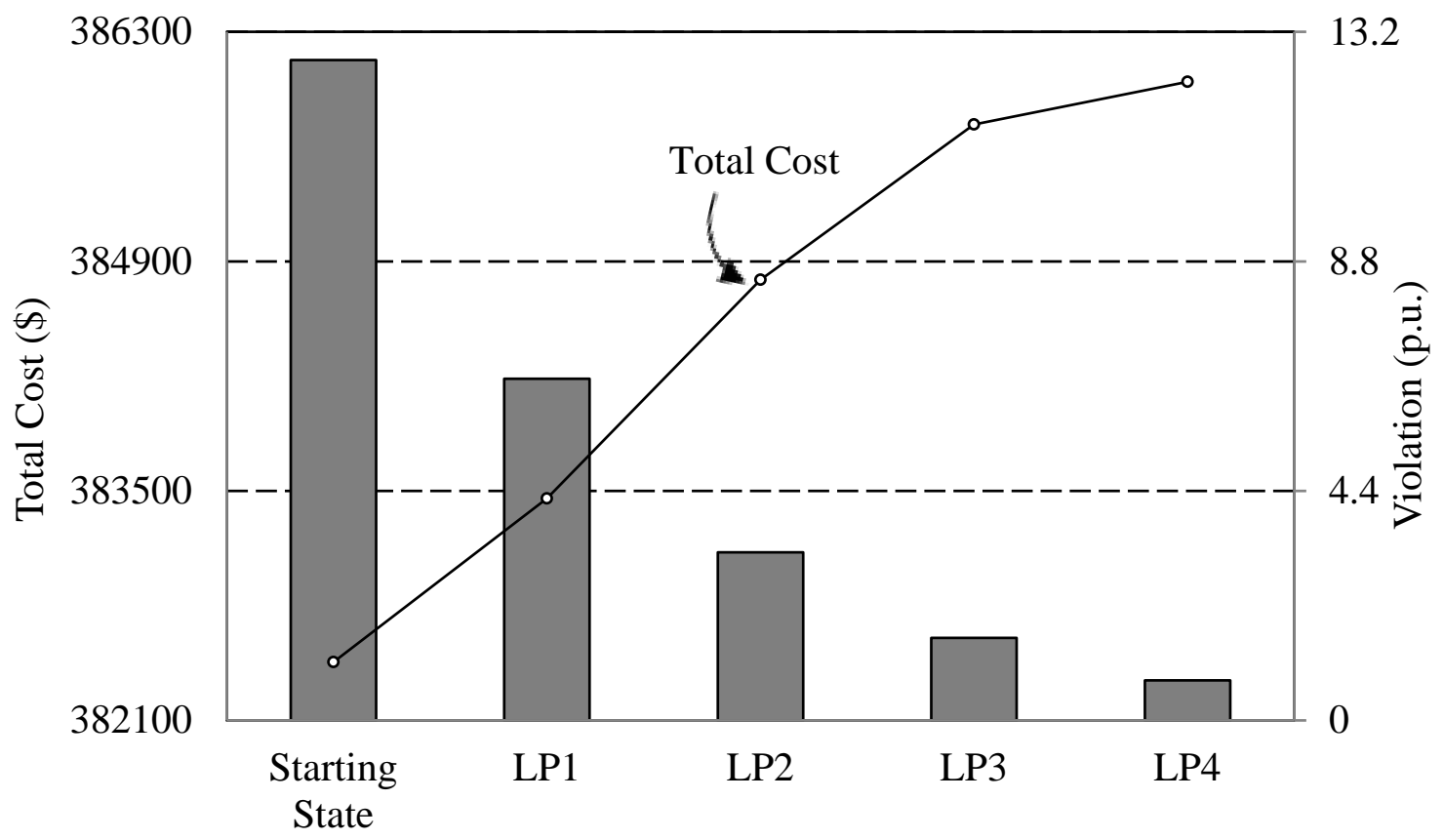

Fig. 4.10 Convergence characteristic of the 57-bus with one line out 
With one line outage, there is not enough capacity to transfer power out from bus \#8. The DC-SCUC cannot be solved with this line capacity. Similar to the third case of the 6-bus system, the starting state is obtained by increasing the line capacity by 1.5 times (300 MVA). After solving the power balance equations and OPF, the transmission line violation is still large. After four LPMOVEs, the largest violation on the transmission lines can be reduced to $5 \%$ overload, as shown in Figure 4.10.

Table 4.3 presents the problem details for the IEEE 57-bus system with transmission outage in the 24-hour study duration.

Table 4.3 Details of 57-bus System with transmission outage in 24 hours

\begin{tabular}{l|l}
\hline System & IEEE 57-bus \\
\hline Computer & Windows/64-X86 \\
\hline Constraints & 13,503 \\
\hline Variables & 7,105 \\
\hline
\end{tabular}

\begin{tabular}{l|l}
\hline Integer Variables & 1,248 \\
\hline LPMOVEs & 4 \\
\hline Total Schedule Cost (\$) & $385,996.03$ \\
\hline Executing Time (Sec) & 200 \\
\hline
\end{tabular}

Line power flows for the starting state and the final LPMOVE are compared in Table 4.4. The overflow ratio is reduced from $20-40 \%$ to $2-4 \%$ of the total transmission capacity on the lines. 
Table 4.4 Transmission line MVA flows of 57-bus system for two states

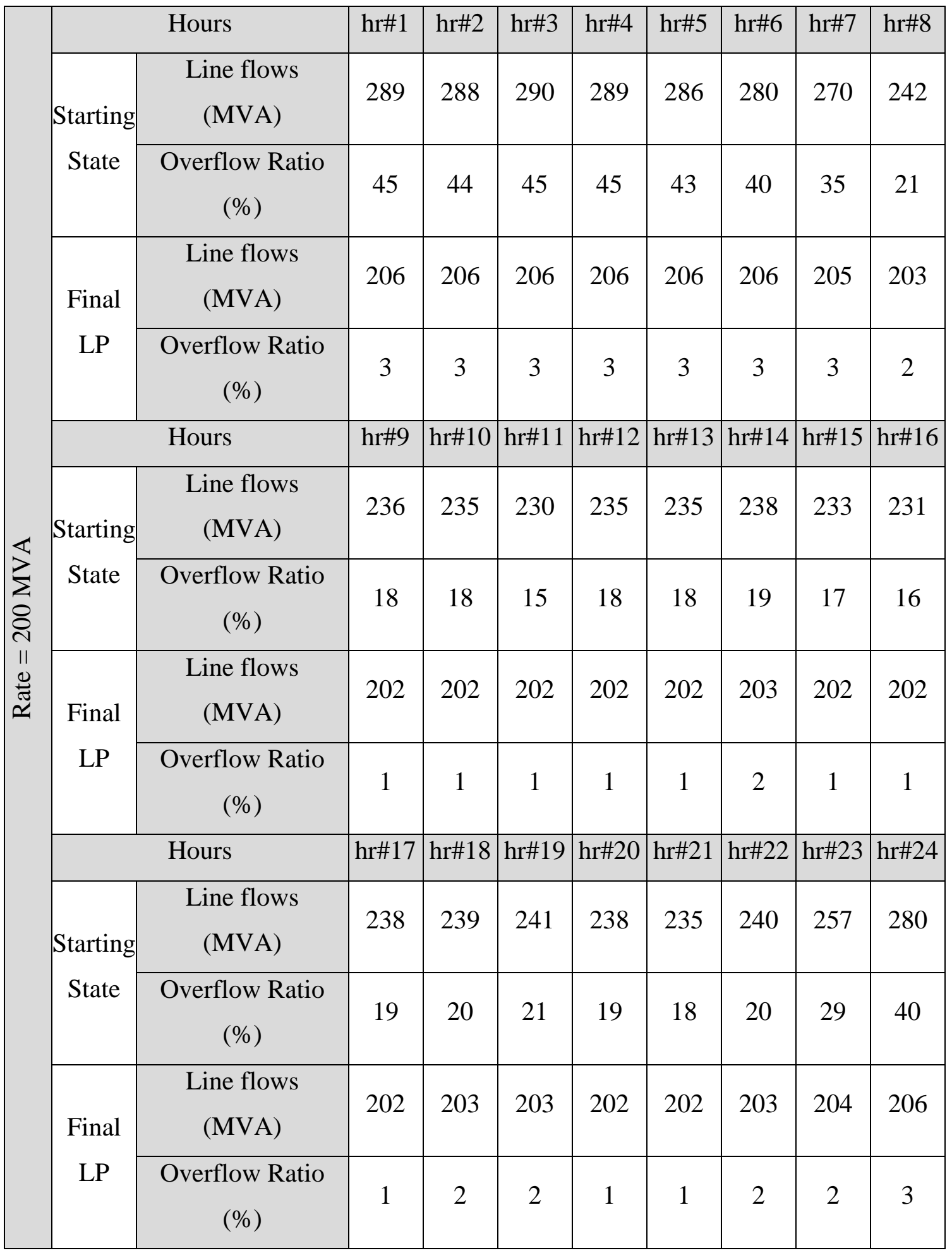




\subsubsection{IEEE 118-bus system}

The same IEEE 118-bus test system that was used in Chapter 3 is also used for this case study. The system has 54 generators, 65 load buses, and 186 transmission lines. By inspecting the system data, it is shown that bus \#10 is connected through only one transmission line to bus \#9. In addition, a generator with large capacity is installed on bus \#10.

One more transmission line is added between bus \#10 and bus \#9 to build a multiple-line connection (2 lines) for testing purposes. The capacity of each line is set as 200 MVA. For simplicity, the lower and upper limits of voltage magnitude are taken as 0.95 p.u. and 1.10 p.u. for all buses. Similar to the tests for the IEEE 57-bus system, two different states are studied using the proposed successive fuzzy MILP method and are described in detail below.

1) No transmission line outage

The DC-SCUC and AC power balance equations are solved for the starting state. Without transmission outage on the lines between buses \#10 and \#9, the total capacity of this connection is 400 MVA. With sufficient transmission capacity for the generator connected to bus \#10, there is no violation on the lines between bus \#10 and bus \#9. The total cost has been minimized for this system by the proposed method as shown in Figure 4.11. 


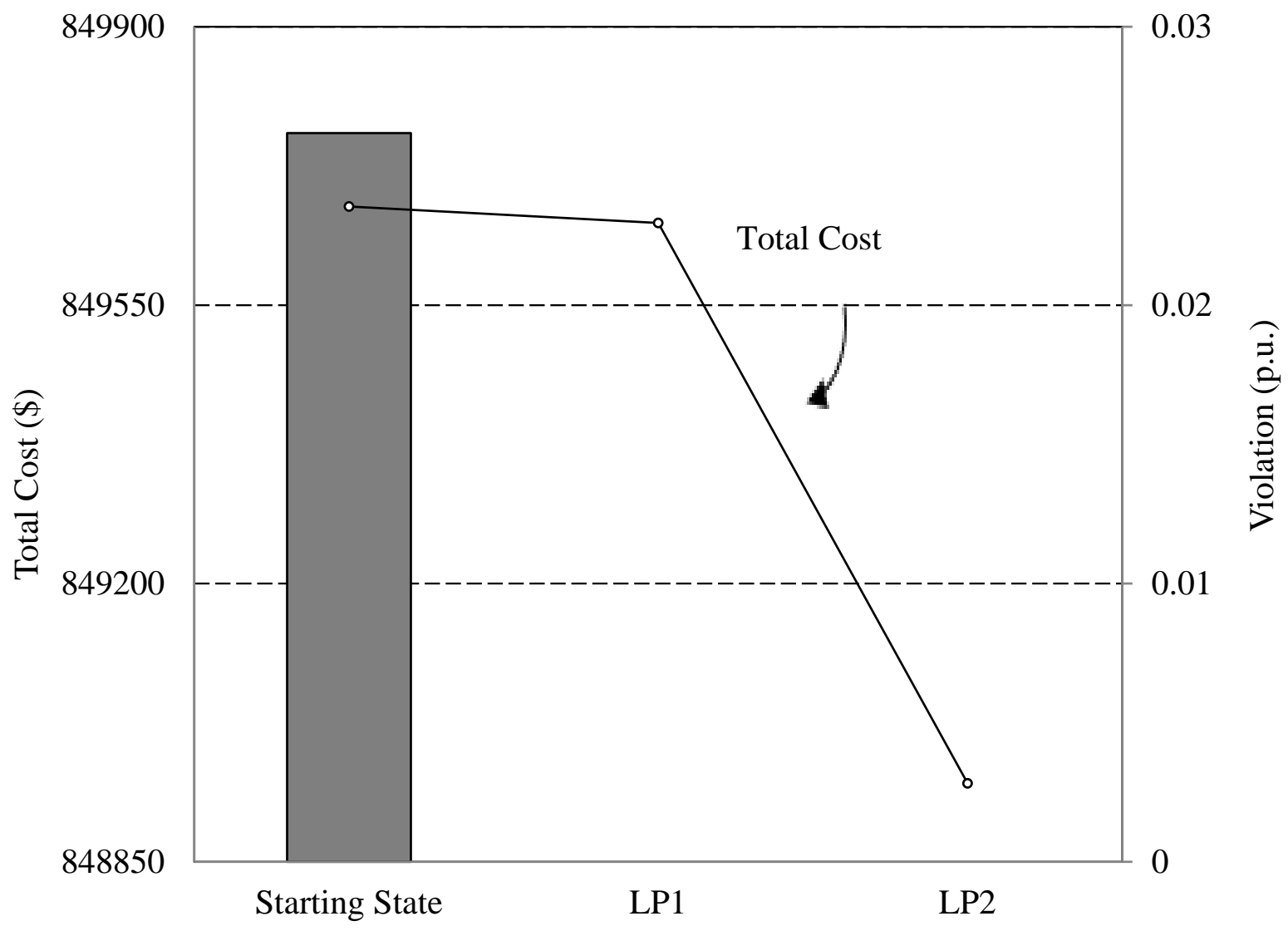

Fig. 4.11 Convergence characteristic of the IEEE 118-bus without outage

2) One transmission line is out of service

The case study with one line outage has been examined for the IEEE 118-bus system. The transmission capacity between buses \#10 and \#9 drops to 200 MVA and violations appeared in the remaining transmission line between these buses. 
With one line outage, there is an insufficient transmission capacity to transfer power through the line between bus \#10 and bus \#9, and huge line flow limit violations appear in the system. The largest line power flow between the buses \#10 and \#9 in 24 hours is calculated as 278 MVA, which is $39.4 \%$ overflow. After 4 LPMOVEs, the largest violation in 24 hours drops to 210 MVA, which is $4.9 \%$ over the line capacity. The convergence characteristics of the total cost and line violations are shown in Figure 4.12.

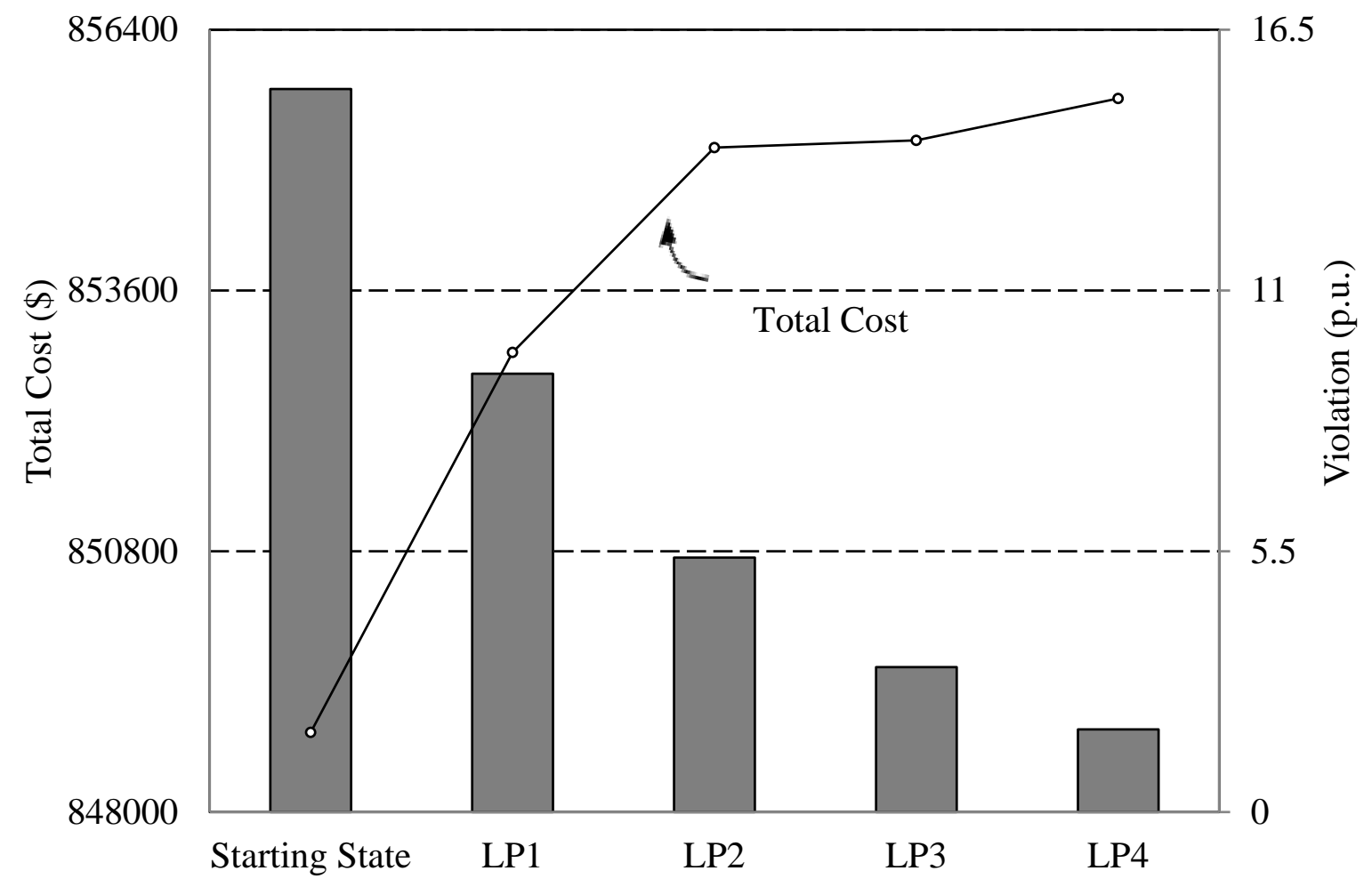

Fig. 4.12 Convergence characteristic of the IEEE 118-bus with one line out 
Table 4.5 presents the optimal schedule with a total operation cost of $\$ 855,660.89$ for 24 hour study duration with the IEEE 118-bus system. A summary for this chapter is given in the next section.

Table 4.5 Details of 118-bus System with transmission outage in 24 hours

\begin{tabular}{l|l}
\hline System & IEEE 118-bus \\
\hline Computer & Windows/64-X86 \\
\hline Constraints & 28,532 \\
\hline Variables & 14,737 \\
\hline
\end{tabular}

\begin{tabular}{l|l}
\hline Integer Variables & 2,592 \\
\hline LPMOVEs & 4 \\
\hline Total Schedule Cost (\$) & $\$ 855,660.89$ \\
\hline Executing Time (Sec) & 408 \\
\hline
\end{tabular}

\subsection{Chapter Summary}

This chapter proposes a successive MILP algorithm for AC security constrained unit commitment (AC-SCUC) with fuzzy enforcement of security constraints. The AC-SCUC challenge, a nonlinear mixed integer optimization problem, is linearized to form a MILP model while retaining hourly and inter-temporal constraints. The MILP model is transformed into a fuzzy MILP model to overcome infeasibility.

On solving the fuzzy MILP model, the algorithm yields optimal incremental changes to the schedule. The process of setting up the fuzzy MILP model, solving it, and updating the schedule and solution of 24 sets of power balance equations comprises one LPMOVE step. 
These LPMOVE steps are iterated successively until the optimum is reached and all violations are removed or minimized (for a weak network).

The method is shown to work well on small (6-bus), medium (57-bus), and large (118-bus) sized systems with correspondingly more complex transmission networks. It produces optimal solutions in less than 7 minutes for larger systems with insufficient transmission capacity, especially during transmission contingency or planed outage.

In this chapter, the SCUC with AC security constraints is successfully solved with the proposed method. WEGs are not considered in this proposed method. To account for the integration of WEGs, a fast security and risk constrained probabilistic unit commitment method using the TAD model of WEGs is proposed in the next chapter. 


\section{CHAPTER 5}

\section{FAST SECURITY AND RISK CONSTRAINED}

\section{Probabilistic Unit Commitment Method Using}

\section{THE TAD MODEL OF WIND GENERATORS}

The TAD model of WEGs and its use in the OPF algorithm are discussed in Chapters 2 and 3 respectively. Due to the geometric similarity between isosceles triangles and normal probabilistic distribution curve, this TAD model of WEGs can closely represent the probabilistic nature of power output from WEGs. The TAD model is described using a set of simple linear equations that lends itself to flexible and efficient computation in comparison to the more complex nonlinear model of normal probabilistic distribution function. Moreover, the SCUC problem with AC network security constraints (AC-SCUC) studied in the previous chapter can be formulated into a fuzzy MILP problem and solved successively to find the optimal generation schedule with the minimum total cost and the least system violations. In this chapter, the TAD model is used to represent the probabilistic nature of WEGs in the SCUC problem. This will enable the SCUC formulation to account for the uncertainty of WEGs using linear models to accurately quantify the probabilistic behavior of WEGs. The details of this proposed method are provided in the following sections. 


\subsection{Security and Risk Constrained Probabilistic UC Algorithm}

A security and risk constrained probabilistic unit commitment (SRCPUC) algorithm is proposed using the TAD model of WEGs taking the transmission network constraints into account. The proposed algorithm is solved using MILP technique. CEGs are committed to provide sufficient reserve to counter the risk from the uncertainty of output from WEGs and possible N-1 contingencies. This risk is quantified by an EENS function and computed using the TAD model. For the purposes of comparison, the classical enumeration technique is used to produce scenarios of real power output of WEGs and to verify the result of the proposed SRCPUC algorithm with the TAD model of WEGs.

Similar to the procedure in Chapter 4, the SRCPUC problem with WEGs is solved in two steps:

1) In the first step, a deterministic SCUC formulation is solved with mean values of forecasted WEG power output. This MILP formulation yields a base case solution (starting state). In this solution process, reserves to counter EENS are assigned considering mean power output from WEGs.

2) In the second step, an incremental formulation of SRCPUC is developed to minimize scheduling costs while also minimizing EENS. This incremental formulation is successively solved while updating control and dependent vectors until the optimal solution is achieved.

\subsubsection{Deterministic MILP Formulation of SCUC with WEGs}

The objective is to minimize the total cost (TC) including the operating cost, the reserve cost, 
the start-up cost of CEGs and the energy cost of WEGs, as shown in the following equations.

Objective: minimize TC for 24 hours:

$$
\begin{aligned}
\mathrm{TC}= & \sum_{t} \sum_{n \in C G}\left[a c_{n} \cdot U_{t n}+\left(\sum_{m} b c_{n m} \cdot P M_{t n m}\right)+r c_{n} \cdot S R_{t n}+S c_{n} \cdot S U_{t n}\right] \\
& +\sum_{t} \sum_{n \in W G}\left(a w_{n}+b w_{n} \cdot P G_{t n}\right)
\end{aligned}
$$

The cost of wind power is added in the objective function, which is not considered in (4.1) in Chapter 4. This SCUC formulation is constrained by hourly and inter-temporal constraints, referring to equations (4.2) - (4.21) in Chapter 4. In order to show the influence of involving WEGs into the SCUC process and to avoid complexity, the AC security constraints are simplified into DC security constraints. A few assumptions are made for DC transmission network constraints:

1) The system is lossless;

2) The magnitude of voltages at all buses are equal to 1.0 p.u.; and

3) Reactive power is ignored.

The deterministic SCUC challenge with DC security constraints can be formulated as a MILP task [6]. Only simplified constraints, comparing to chapter 4, are given as the following:

DC Power balance: $\quad \sum_{n} P G_{n}=\sum_{i} P D_{i}$ 
The DC power balance equations are derived from equations (4.2) and (4.3) using assumptions above. Reactive power limits (4.14), voltage magnitude limits (4.15), and voltage phase angle limits (4.16) are ignored in this case.

$\underline{\text { DC transmission line flow limit: }} \underline{P L_{l}} \leq \sum_{i}\left[\Theta_{k i} \cdot\left(\sum_{n \in G I i} P_{t n}-P D_{t i}\right)\right] \leq \overline{P L_{l}} \quad \forall t, l$

Using the DC load flow method, real power line flows can be computed using net real power injection at buses. The DC transmission line constraint is linear by nature. The matrix $\Theta_{k i}$ is constructed from the system susceptance matrix as detailed in Appendix A. The upper and lower bounds of real power flows are taken as $\pm \overline{S F_{l}}$ (AC line flow MVA limits).

In addition, power limits and constraints for the output of WEGs are enforced in the SCUC problem. The generation limits of WEGs are assessed using same method in Chapter 2 (i.e., equations 2.5 and 2.6, which are functions of mean values and standard deviations). For a real power output from WEGs equaling $P G_{t n}$, one may calculate an EENS value to quantify the risk of uncertainty, for which details are provided in Chapter 2 (i.e., equations 2.1 to 2.3). In this case, $P G_{t n}$ is set to equal the mean value $\left(\overline{\overline{P G_{t n}}}\right)$ without considering its probabilistic behavior.

Power limit WEGs: $\quad P G_{t n}=\overline{\overline{P G_{t n}}} \quad \forall t, n \in W G$

EENS:

$$
E_{t n}=c p_{t n} \overline{\overline{P G_{t n}}} \forall t, n \in W G
$$


To solve objective (5.1), CEGs must provide sufficient reserve to carry the risk resulting from the uncertainty of WEGs, which is not considered in (4.10) in Chapter 4, and possible N1 contingencies. One portion of demand, quantifying the unreliability of equipment ( $\mathrm{N}-1$ contingencies) is also covered by total system reserves. Quick-start units are supplemental reserve capacity sources to be scheduled in (5.6).

\section{$\underline{\text { Total system reserve constraint: }}$}

$$
\sum_{n \in C G} S R_{t n}+\sum_{n \in G 10}\left(1-U_{t n}\right) \cdot P 10_{n} \geq \alpha \cdot \sum_{i} P D_{t i}+\beta \cdot \sum_{n \in W G} E_{t n} \forall t
$$

Using the same work conducted in Chapter 3, the values $\alpha$ and $\beta$ are chosen as 0.1 and 0.6. Unit status $U_{t n}$ and start-up status $S U_{t n}$ are restricted as integer variables with the values of 0 or 1.

After solving the MILP SCUC formulation with forecasted wind power, a base case solution (starting state) is obtained that considers wind generation equal to the forecasted values. The inclusion of WEGs' output into the schedule influences the total cost directly in objective (5.1) and indirectly by altering the value of EENS (5.5), which in turn necessitates the additional procurement of adequate reserves in (5.6). Therefore, making wind generation a problem variable by allowing it to vary and including (5.5) into (5.1) causes the model to become an inter-temporal nonlinear mixed integer optimization challenge. This problem, encompassing (5.1) and (5.5), is solved using SLP technique as outlined below. 


\subsubsection{Formulation of SRCPUC with TAD model}

The SCUC formulation of (5.1) is transformed into an incremental formulation of SRCPUC by using the incremental linear relation between changes in real power output of WEGs and changes in the EENS value. This relation captures the probabilistic behavior of WEGs. Also, reserves are purchased from CEGs to counter possible risk. Accordingly, the optimization process chooses the best values of WEGs' outputs to minimize the total cost, taking into account the benefits of WEGs and their risks. The objective of the SRCPUC formulation is to minimize change in TC in terms of incremental variables $(\Delta \mathrm{TC})$.

Objective: minimize:

$\Delta \mathrm{TC}=\left[\begin{array}{l}\sum_{\mathrm{t}} \sum_{\mathrm{n} \in \mathrm{CG}}\left[\mathrm{ac}_{\mathrm{n}} \cdot \Delta \mathrm{U}_{\mathrm{tn}}+\sum_{\mathrm{m}}\left(\mathrm{bc}_{\mathrm{nm}} \cdot \Delta \mathrm{PM}_{\mathrm{tnm}}\right)+\mathrm{rc}_{\mathrm{n}} \cdot \Delta \mathrm{SR}_{\mathrm{tn}}+\mathrm{Sc}_{\mathrm{n}} \cdot \Delta \mathrm{SU}_{\mathrm{tn}}\right] \\ +\sum_{\mathrm{t}} \sum_{\mathrm{n} \in \mathrm{WG}} \mathrm{bw}_{\mathrm{n}} \cdot \Delta \mathrm{PG}_{\mathrm{tn}}\end{array}\right]$

Subject to the following incremental linear equality and inequality constraints:

DC power balance: $\quad \sum_{n} \Delta P G_{t n}=0 \forall t$

By ignoring transmission losses and considering no change on demand, the sum of increment of real power generation must remain unchanged. Total real power generation from all generators must be bounded within their respective limits.

Power limit WEGs: $\quad \underline{P G_{n}} \leq\left(P G_{t n}+\Delta P G_{t n}\right) \leq \overline{P G_{n}} \quad \forall t, n \in W G$ 
EENS:

$$
\Delta E_{t n}=J E P_{t n} . \Delta P G_{t n} \forall t, n \in W G
$$

The sensitive matrix $J E P_{t n}$ is computed by the TAD model, using the current state of wind power output.

System reserve constraint:

$$
\sum_{n \in C G}\left(S R_{t n}+\Delta S R_{t n}\right)+\sum_{n \in G 10}\left(1-U\left({ }_{t n}+\Delta U_{t n}\right)\right) \cdot P 10_{n} \geq \alpha \cdot \sum_{i} P D_{t i}+\beta \cdot \sum_{n \in W G}\left(E_{t n}+\Delta E_{t n}\right) \forall t
$$

$\underline{\text { DC line flow constraint: }} \quad 0 \leq \sum_{i}\left[\Theta_{k i} \cdot\left[\sum_{n \in G I i}\left(P_{t n}+\Delta P_{t n}\right)-P D_{t i}\right]\right] \leq \overline{P L_{l}} \quad \forall t, l$

In addition, constraints (4.26)-(4.33) and (4.39)-(4.43) in Chapter 4 are enforced in the proposed SRCPUC algorithm. Those constraints are mainly considered for CEGs, as they are not directly linked with WEGs.

It is important to note that (5.10) relates the output of WEGs to total EENS. This EENS is used as an element to compute the net reserves required in (5.11). These reserves appear in objective (5.7) and affect the cost of the schedule. Hence, when a larger amount of wind is scheduled, it would lead to a bigger EENS value, requiring a larger amount of reserves and creating a higher cost.

The same assumptions are made for DC transmission network security constraints as in the last section. Restrictions are applied on $\left(U_{t n}+\Delta U_{t n}\right)$ and $\left(S U_{t n}+\Delta S U_{t n}\right)$ to ensure integer variables with the values of 0 or 1 . This formulation of SRCPUC with the TAD model can be readily solved using the MILP technique. 
The SRCPUC problem with the TAD model is solved successively until the TC can no longer be significantly reduced. The algorithm of the proposed method is given in next section.

\subsection{Algorithm of SRCPUC}

Figure 5.1 shows the algorithm of the proposed SRCPUC problem with the TAD model. The steps of this problem are as follows:

1) With forecasts of wind generation, the SCUC model (5.1) is solved to establish the base case (starting state).

2) An incremental formulation of SRCPUC with TAD model of WEGs is set up using the base case from step 1.

3) The incremental formulation is solved using the MILP technique, obtaining the optimal changes in integer and continuous variables.

4) All variables are updated and the costs are computed. It is checked if change in TC is significant.

5) Steps 2 to 4 constitute an LPMOVE. These LPMOVEs are successively repeated until the total cost can no longer be significantly reduced.

Several systems are chosen and tested using the proposed SRCPUC algorithm with the TAD model. The results are discussed in the following sections. 


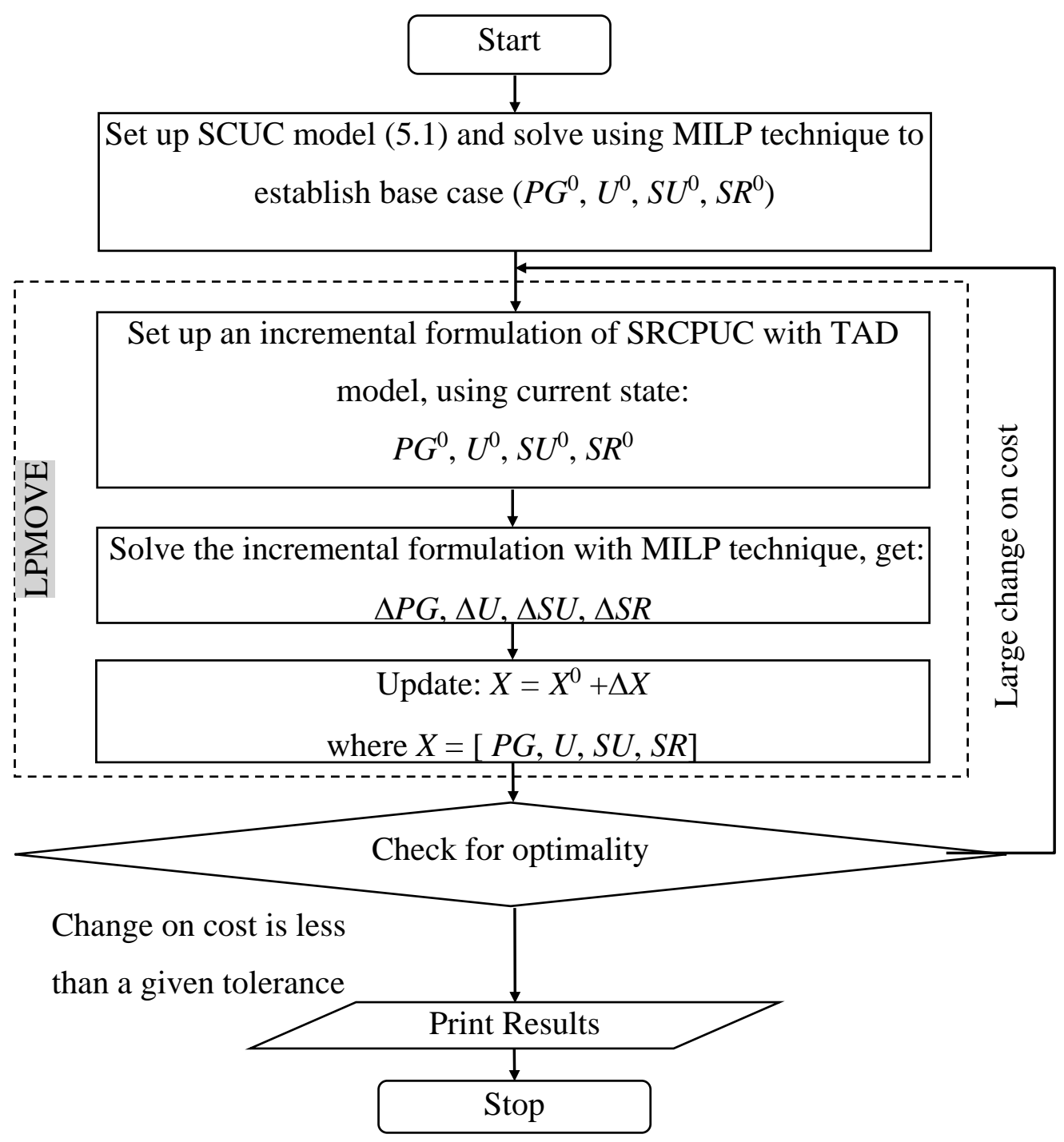

Fig. 5.1 Flowchart of the proposed SRCPUC algorithm with TAD model 


\subsection{Test Results and discussions}

In this section, the results of the test cases are presented. Systems of different sizes are chosen as follows:

1) A 6-bus system with 3 CEGs and 1 WEG over a 3-hour time span.

2) The IEEE 118-bus system with 53 CEGs and 1 WEG on a 3-hour time horizon.

3) The IEEE 118-bus system with 51 CEGs and 3 WEGs for 24 hours.

The small system is chosen to illustrate key features of the method and demonstrate its benefits, whereas the large system is chosen to show how the method performs for a larger and more complicated system with multiple WEGs.

To test the proposed method, a classical technique taking into account nonlinear PDF of normal probabilistic distribution was also developed and implemented. The method is explained in detail in Appendix A. In brief, the method considers several combinations of WEG outputs. For each combination, this method uses the nonlinear PDF of normal probabilistic distribution to computes an EENS value and solves the optimization challenge of (5.1). This is called classical enumeration technique. By comparing all solutions obtained using classical enumeration technique, the least costing solution is chosen as the optimal solution. All results computed using the proposed SRCPUC method with the TAD model are compared with those obtained using the classical enumeration technique. 


\subsubsection{6-bus system with 1 WEG in 3 hours}

The same 6-bus system has also been used in Chapter 4 to solve the AC-SCUC problem. The diagram and complete system data is given in Appendix B. The system has three CEGs and one WEG supplying three loads. The WEG is installed at the bus \#4. The system has a transmission network with 7 lines.

In the 6-bus system, the CEG \#1 is the least expensive unit and the CEG \#3 is most expensive. The energy price of the WEG is set between the costs of CEG \#2 and CEG \#3 for test purposes. The transmission capacity of line \#4, which connects bus \#2 and bus \#4, is constrained at 55 MW. When examining a normal distribution, the standard deviation $(\sigma)$ of the short-term wind power forecast is taken as $10 \%$ of the mean output of the WEG for the three-hour duration of this test.

Table 5.1 shows the optimized generation scheduling solution from the proposed SRCPUC algorithm with the TAD model of WEGs. Comparing the final optimized result to the starting state, one can make the following observations:

1) The least expensive unit, CEG \#1, is scheduled to its full capacity in all three hours.

2) Owing to the transmission constraint of line \#4, the generation of CEG \#2 is limited to 55 MW in hour \#3, but the forecast output (mean value) of the WEG is not sufficient to meet the supply demand. Therefore, the most expensive unit, CEG \#3, must be turned on at the starting state. 
Table 5.1 Generation scheduling and total cost in the proposed SRCPUC Algorithm

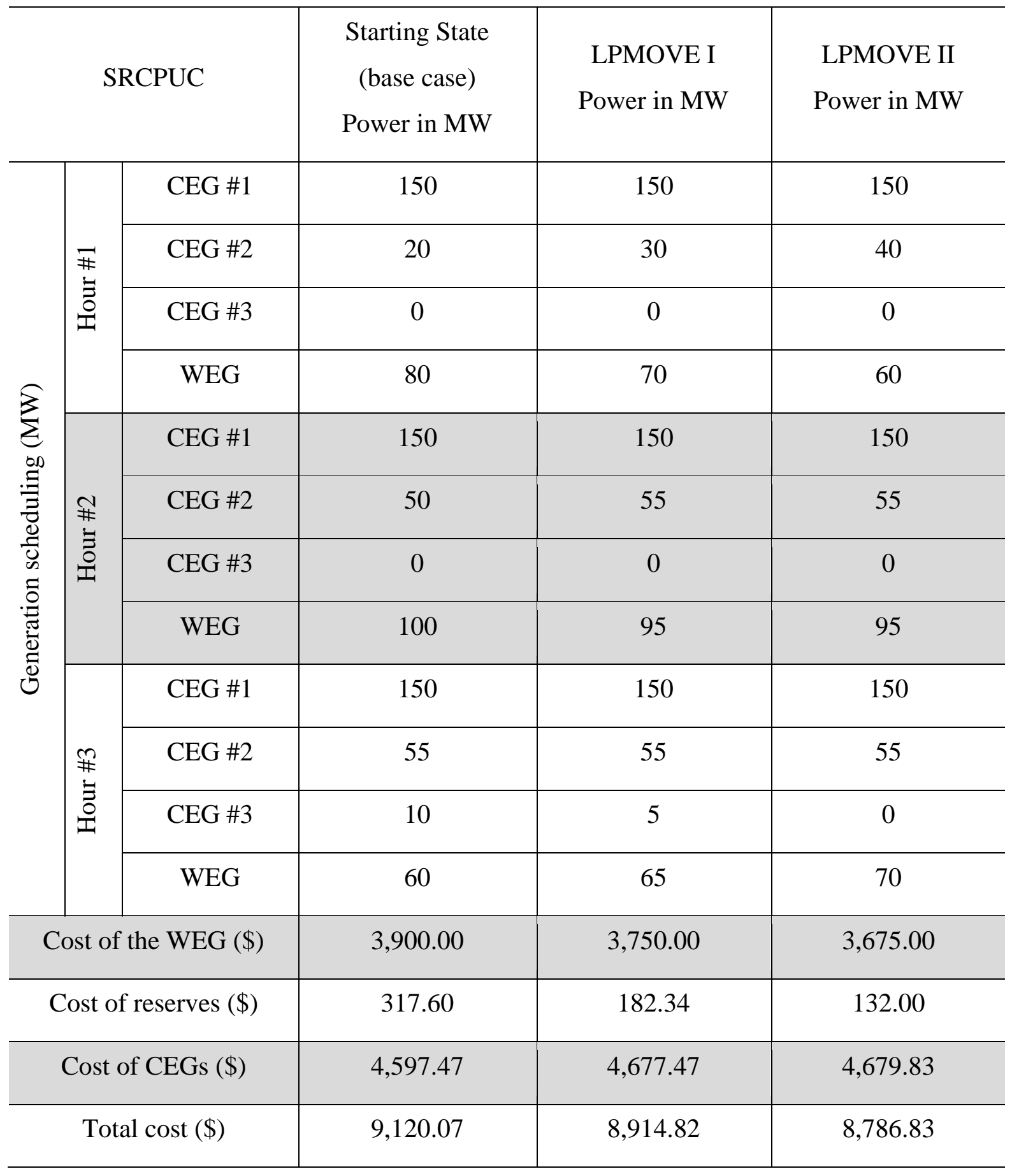


3) Energy from the WEG is cheaper than that of CEG \#3, but more expensive than that of CEG \#2. The WEG is curtailed in hour \#1 and hour \#2, but then has more power scheduled in hour \#3. The WEG is operated below its mean values in hours \#1 and \#2, but above the mean value in hour \#3.

4) The generation cost of CEGs increases but the total cost is reduced. Less power is scheduled from the WEG and reserve cost reduces.

The above results are compared with those of the classical enumeration technique. A detailed explanation of the classical enumeration technique and its usage in this paper is provided in Appendix A.

The final generation schedule and total cost obtained by the proposed SRCPUC algorithm with the TAD model of WEGs are compared with the results of the classical enumeration method in Tables 5.2 and 5.3. The normal distribution curves of the WEG are divided equally into 11 segments. The total number of scenarios is $\left[(11)^{1}\right]^{3}(=1331)$, referring to Appendix A. These scenarios are used to generate output values of WEGs. A thousand three hundred and thirty one (1331) sets of generation schedules are computed and compared to find the least cost scenario. The generation schedule of the scenario with the lowest cost is identified and is then verified by comparing these results to the proposed SRCPUC algorithm with the TAD model of WEGs. 
Table 5.2 Results of proposed SRCPUC method with TAD model of WEGs compared to the classical enumeration method (MW)

\begin{tabular}{c|c|c|c|c|c|c}
\hline \multirow{2}{*}{ Method } & \multicolumn{2}{|c|}{ Time: Hour \#1 } & \multicolumn{2}{c|}{ Time: Hour \#2 } & \multicolumn{2}{c}{ Time: Hour \#3 } \\
\cline { 2 - 7 } & $\begin{array}{c}\text { Classical } \\
\text { (MW) }\end{array}$ & $\begin{array}{c}\text { SRCPUC } \\
\text { Method (MW) }\end{array}$ & $\begin{array}{c}\text { Classical } \\
(\mathrm{MW})\end{array}$ & $\begin{array}{c}\text { SRCPUC } \\
\text { Method (MW) }\end{array}$ & $\begin{array}{c}\text { Classical } \\
(\mathrm{MW})\end{array}$ & $\begin{array}{c}\text { SRCPUC } \\
\text { Method } \\
\text { (MW) }\end{array}$ \\
\hline CEG \#1 & 150 & 150 & 150 & 150 & 150 & 150 \\
\hline CEG \#2 & 40 & 40 & 55 & 55 & 53 & 55 \\
\hline CEG \#3 & 0 & 0 & 0 & 0 & 0 & 0 \\
\hline WEG & 60 & 60 & 95 & 95 & 72 & 70 \\
\hline Demand & \multicolumn{2}{|c|}{250} & & 300 & & 175 \\
\hline
\end{tabular}

Table 5.3 Costs, EENS, reserve, and execution times of the 6-bus system

\begin{tabular}{c|c|c|c|c}
\hline $\begin{array}{c}\text { Wind power } \\
\text { representation }\end{array}$ & Total cost (\$) & EENS (MWh) & $\begin{array}{c}\text { Reserve** } \\
(\mathrm{MW})\end{array}$ & $\begin{array}{c}\text { Execution time } \\
\text { (seconds) }\end{array}$ \\
\hline Classical & 8836.96 & 100.05 & 142.53 & 272.89 \\
\hline TAD model & 8786.83 & 96.34 & 140.39 & $0.49^{*}$ \\
\hline
\end{tabular}

*Including the time (0.16 seconds) to produce the starting state

** Total reserve required without considering EENS is $82.5 \mathrm{MW}$ 
The proposed SRCPUC algorithm with the TAD model of WEGs took 0.49 seconds to solve the problem. It provided a more economical solution through a continuous optimization platform in comparison to discrete 1331 scenarios based optimization that were used in the classical enumeration technique. The SRCPUC method shortens the computational time without lowering the quality of the final solution. Further, the classical enumeration technique took 272.89 seconds to find the solution. Comparing the computational times and optimal solution, benefits of the proposed method are obvious.

\subsubsection{8-bus system with 1 WEG in 3 hours}

The 118-bus system that has already been used in Chapters 3 and 4 is used here for this study with only minor adjustments. These changes are: 1) the price of spinning reserve is set as $10 \%$ of energy cost; 2) one CEG has been replaced by a WEG at bus \#80; 3) three hours duration is considered for this case; 4) the hourly power forecasts of the WEG are assumed as 320 MW, $280 \mathrm{MW}$, and $300 \mathrm{MW}$ in the three hours; 5) standard deviations are taken as $10 \%$ of forecasted values; and 6) The price of energy from the WEG equal a fixed price of $\$ 70$ (aw) and an incremental energy price of \$10/MWh (bw).

Figure 5.2 and Figure 5.3 show the changes of the total cost resulting from these adjustments, as well as the generation cost of CEGs, the cost of the reserve, and the generation cost of the WEG. In summation, the total cost has been reduced by $0.86 \%$ in 2 LPMOVEs of the proposed SRCPUC algorithm with the TAD model of WEGs, even though the generation cost of CEGs increase. Also, less reserve is needed as a result of the reduction in generation by the WEG. 


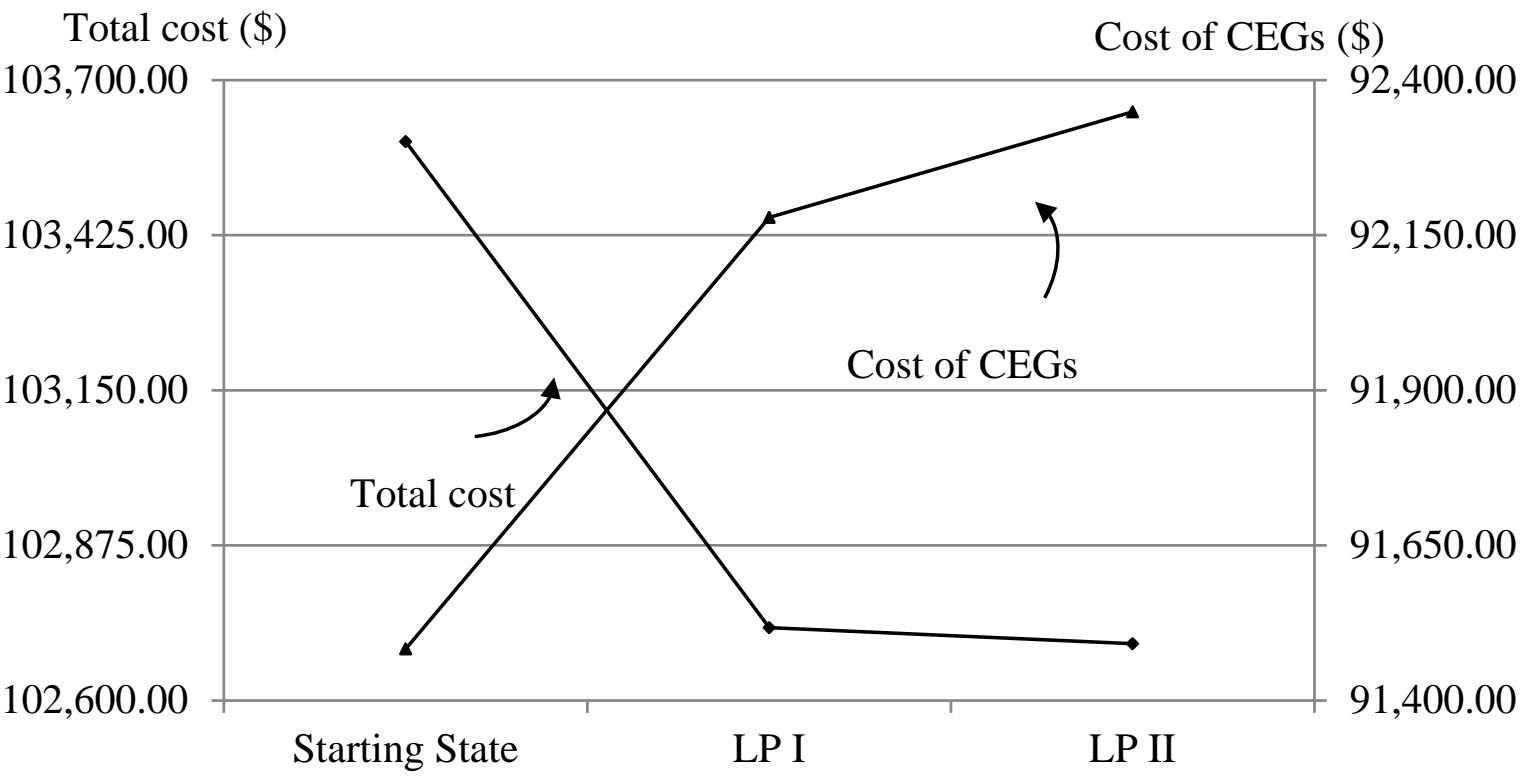

Fig. 5.2 Total cost and cost of CEGs of 118-bus with 1-WEG in 3 hours

Cost of reserve $(\$)$

Cost of the WEG(\$)

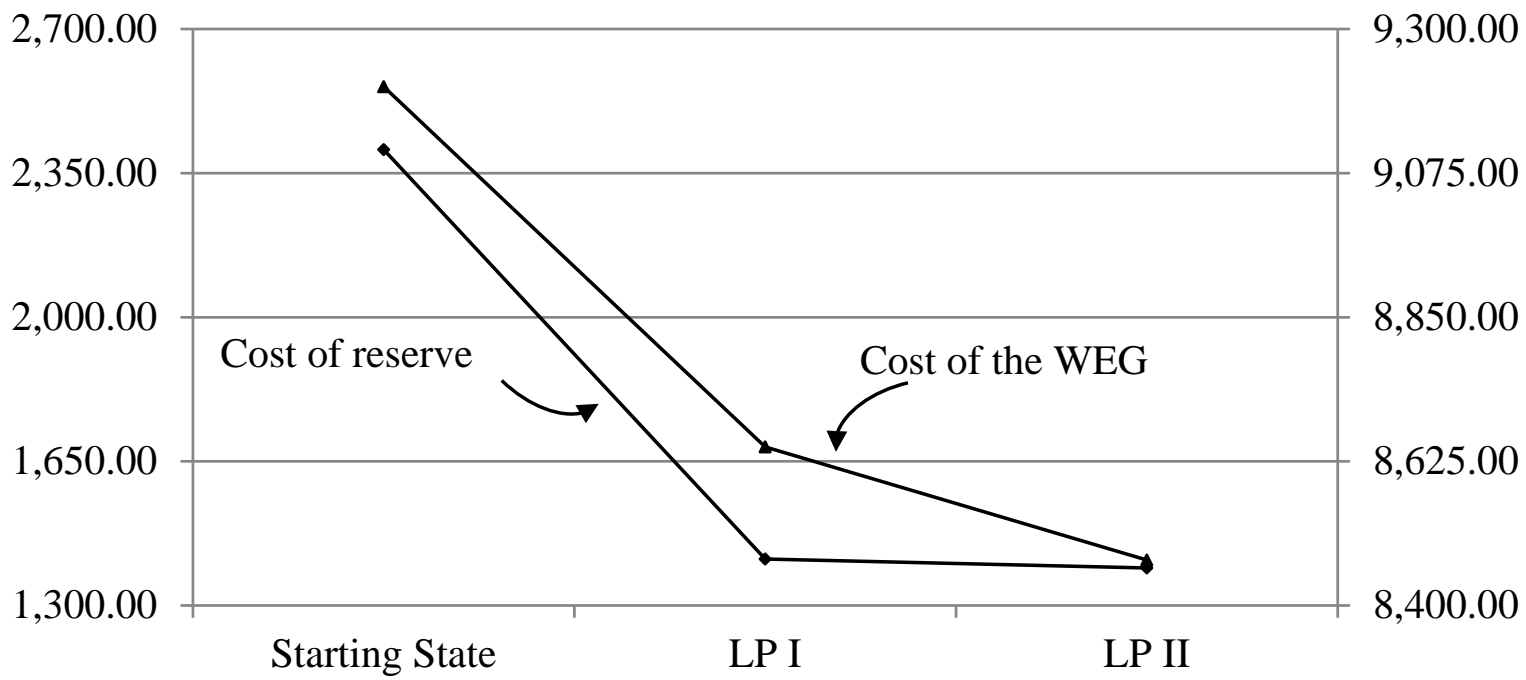

Fig. 5.3 Reserve cost and cost of the WEG of 118-bus with 1-WEG in 3 hours 
For the purpose of comparison, the classical enumeration technique has also been used for the formulation of (5.1). Same as in the case study of the 6-bus system, the normal distribution curves of wind power forecasts are split into 11 segments. One thousand three hundred and thirty one (1331) scenarios are generated and the MILP formulation of SCUC (5) is solved for each scenario. The results of these formulations are compared in Table 5.4.

Table 5.4 Costs and execution times of the 118-bus system with one WEG

\begin{tabular}{c|c|c|c|c}
\hline $\begin{array}{c}\text { Wind power } \\
\text { representation }\end{array}$ & Total cost (\$) & EENS (MWh) & $\begin{array}{c}\text { Reserve** } \\
(\mathrm{MW})\end{array}$ & $\begin{array}{c}\text { Execution time } \\
\text { (seconds) }\end{array}$ \\
\hline Classical & $102,833.56$ & 62.84 & 944.90 & $3,290.08$ \\
\hline TAD model & $102,700.85$ & 203.98 & 1029.59 & $5.86^{*}$ \\
\hline
\end{tabular}

*Including the time (4.96 seconds) to produce the starting state

** Total reserve required without considering EENS is 907.2 MW

Both the proposed SRCPUC algorithm with the TAD model of WEGs and the classical enumeration technique provide comparable optimal solutions, however, the execution time of the classical enumeration technique is about 1 hour whereas the proposed method takes only 5.86 seconds. One could examine more segments in normal distribution curves of WEGs to increase the accuracy of the classical enumeration technique, but this method would exponentially increase the execution time. In contrast, the proposed SRCPUC algorithm with TAD model of WEGs examines a continuous solution domain to provide the optimal solution. 


\subsubsection{8-bus system with 3 WEGs in 24 hours}

In this case study, the number of WEGs is increased to three. Two more CEGs are replaced by WEGs at buses \#10 and \#89 in the IEEE 118-bus system. The price data of these WEGs is given in Appendix B.

In order to apply the classical enumeration technique with the formulation (5.1), one must segment the normal distribution curves of WEGs. If each distribution curve is split into 11 segments, the classical enumeration technique would create $\left(11^{3}\right)^{24}$ scenarios to span across all possible outputs of the three WEGs in 24 hours. This is an extremely demanding computational challenge, which is not possible to implement on a desktop computer. However, the proposed SRCPUC algorithm with the TAD model of WEGs has no such difficulty; it can fully represent wind power probabilistic characteristics within a few seconds.

Independent forecasts of wind power output over a 24-hour time horizon are assumed and

given in Appendix B. The standard deviations are assumed as 10\% of forecasted values for all the three WEGs in all 24 hours. Convergence curves of costs are given in Figure 5.4 and Figure 5.5 .

The details of the tested 118-bus with 3 WEGs are shown in Table 5.5. Starting with the solution of SCUC (5.1), the proposed SRCPUC algorithm with the TAD model of WEGs solves the large complicated problem in 26.16 seconds 


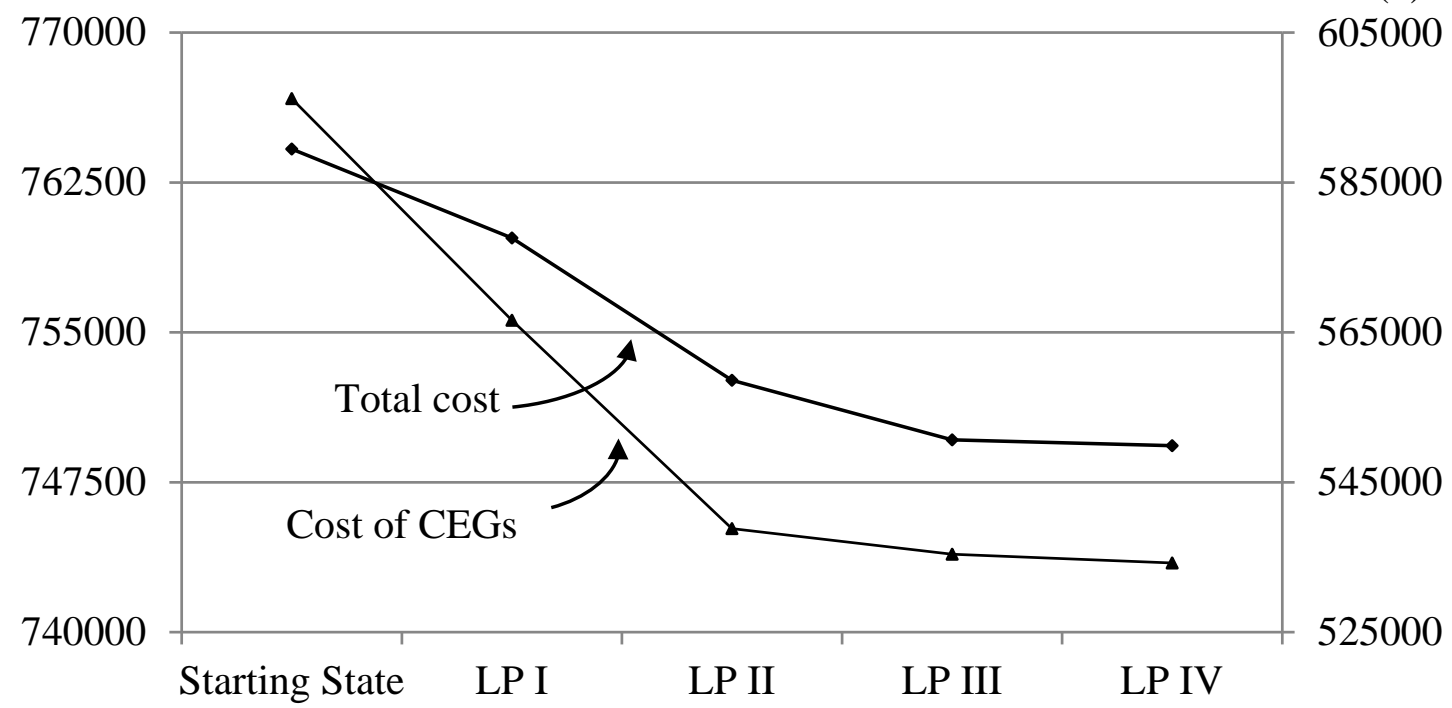

Fig. 5.4 Total cost and cost of CEGs of 118-bus with 3 WEGs in 24 hours

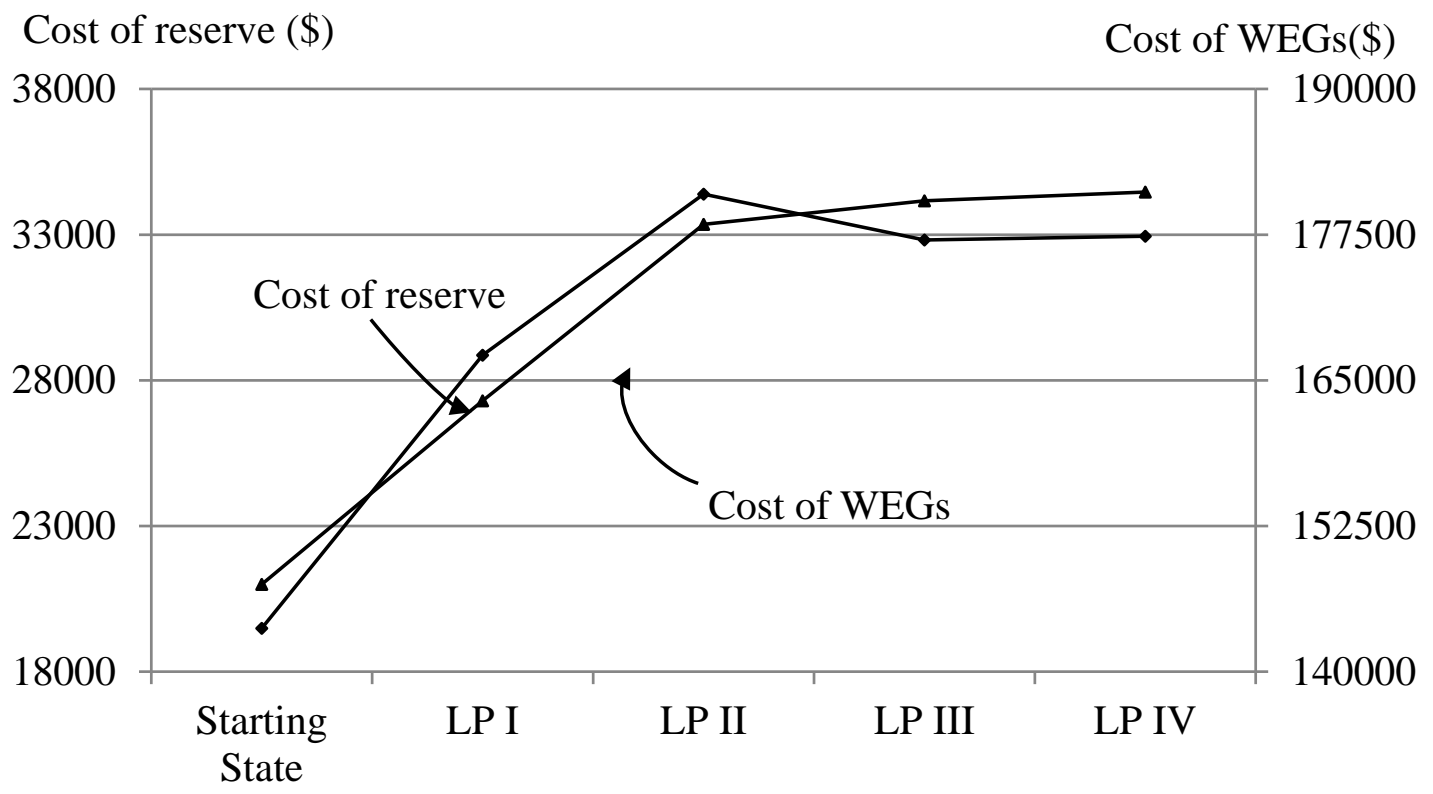

Fig. 5.5 Reserve cost and cost of the 3 WEGs of 118-bus in 24 hours 
Table 5.5 Result of 118-bus System with 3 WEGs in 24 hours

\begin{tabular}{|c|c|c|c|}
\hline Case & 118-bus & Constraints & 14,074 \\
\hline Number of WEGs & 3 & Variables & 7,416 \\
\hline Wind power ratio (\%, hourly average) & 37.7 & Integer Variables & 2,448 \\
\hline Total Schedule Cost (\$) & $749,331.64$ & Execution Time (Sec) & $143.41^{*}$ \\
\hline
\end{tabular}

*Including the time (117.25 seconds) to produce starting state

In this case, the system consumes around 38\% energy from the WEGs in each hour. This demonstrates how the proposed method can make a significant contribution to renewable energies in power systems and is computationally efficient for practical systems with more wind power penetration.

In addition, studies have been conducted for the 118-bus system with different standard deviation values to demonstrate the effects of standard deviation in system scheduling. This test assumes that all WEGs have same standard deviation value and the value of standard deviation changes among $5 \%, 10 \%$, and $15 \%$ of mean values $(\overline{\overline{P G}})$. The total cost and wind energy for 24 hours are computed using three different standard deviation values and the results are given in Figure 5.6. 


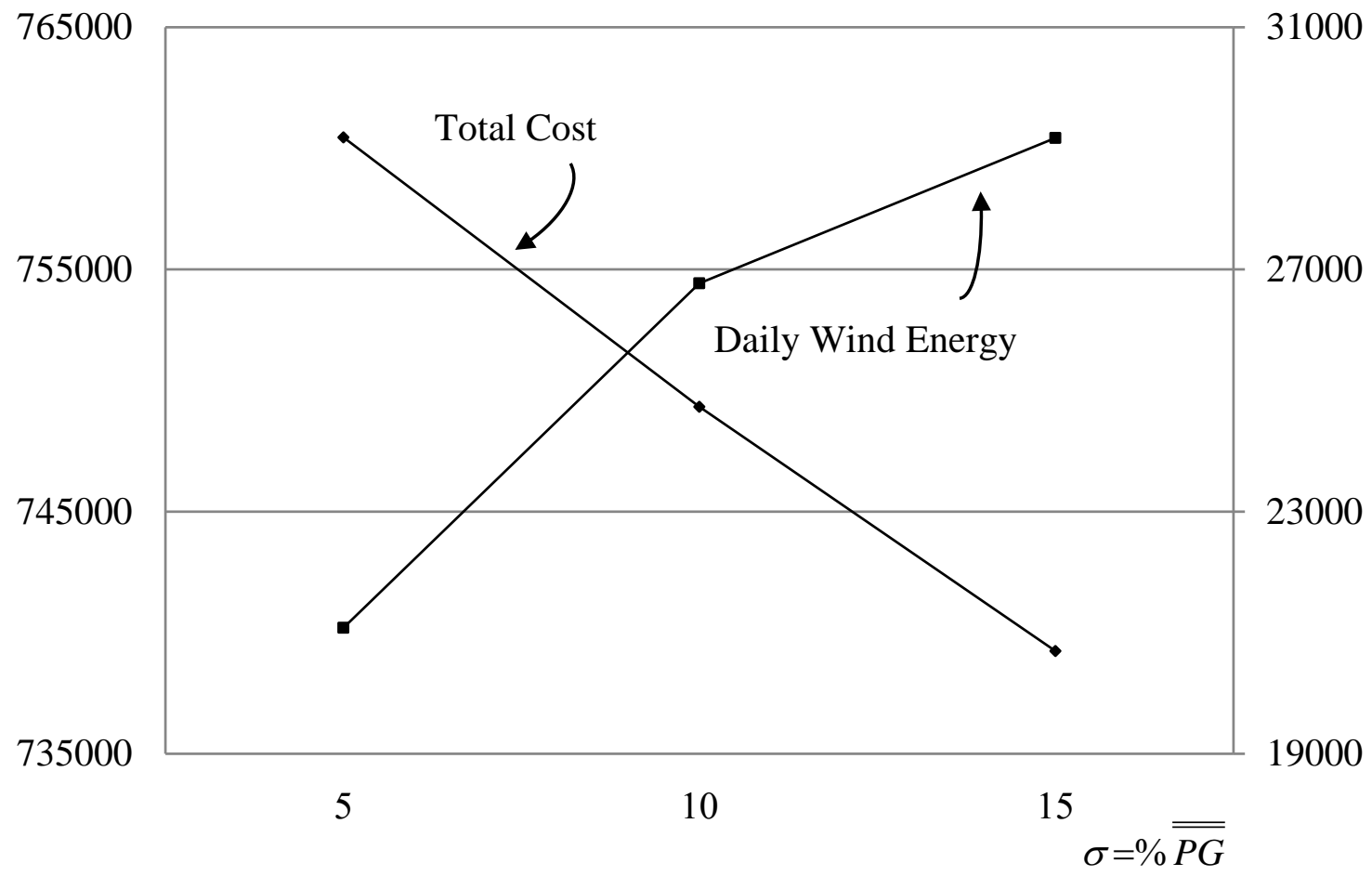

Fig. 5.6 Total cost and wind energy with various standard deviation (118-bus, 3-WEG, 24hour)

With particular prices of energy given in Appendix B for CEGs and WEGs, WEGs are optimally scheduled to operate over their forecasted values in most of the hours across the 24hour planning horizon. For the same amount of power, larger standard deviations correspond to smaller values of EENS. The SRCPUC algorithm allows the system consume more energy from WEGs while reducing the total cost, as shown in Figure 5.6. Indeed, the SRCPUC method is the only form of analytical tool that is capable of investigating the effect of standard deviation of wind forecast on the total schedule costs, which is a significant benefit of this proposed SRCPUC algorithm. 


\subsection{Chapter Summary}

This chapter proposes the SRCPUC algorithm with the TAD model of WEGs as this TAD model can accurately represent probabilistic energy forecasts from WEGs. Furthermore, the TAD model is linearized around the current operating point in the solution algorithm and is used to create the incremental SRCPUC formulation. The proposed SRCPUC algorithm minimizes the total operation cost that includes cost of energy and cost of risk.

The proposed SRCPUC algorithm with the TAD model has been tested on 6-bus and IEEE 118-bus test systems with probabilistic models of WEGs. Comparisons have been made between the performance of the proposed SRCPUC algorithm and the classical enumeration technique applied to the SCUC formulation.

From the results, it can be seen that the proposed SRCPUC algorithm with the TAD model is as accurate as the classical enumeration technique that relies on nonlinear normal probabilistic distribution models. However, the comparison of execution time shows that the proposed method is vastly superior to classical enumeration technique as the execution time does not increase for the proposed method significantly when incorporating more WEGs. In contrast when more WEGs are incorporated, the execution time for the classical enumeration technique grows exponentially.

Using the proposed method, the effects of standard deviation have been examined for large systems. This dissertation has demonstrated the benefit of using the proposed SRCPUC method to compute the effect of standard deviation of wind energy forecast on the total schedule cost. The proposed SRCPUC method with TAD model of WEGS is computationally efficient, 
operationally flexible, and numerically accurate, with the capacity to handle practical transmission systems with large wind power penetration. 


\section{CHAPTER 6}

\section{CONCLUSION AND FUTURE WORK}

General conclusions and the key contributions of this dissertation work are summarized in this chapter. Recommended future research in power system optimization studies as it relates to this dissertation is also discussed.

\subsection{Conclusion and Major Contributions}

The following targets have been achieved and are presented below as key research contributions:

1) The TAD model of WEGs was proposed from and compared against the regular normal probabilistic distribution function. The TAD model provides high degree of accuracy by very closely following the normal probabilistic distribution. The TAD model possesses flexible and simple algebraic features in formulating the EENS function and effectively quantifying the risks caused by uncertainties in energy forecast of WEGs.

2) The TAD model has been linearized and successfully used in an hour-ahead stochastic OPF algorithm. The use of TAD model of WEGs in OPF algorithms enables computationally feasible scheduling and operation of WEGs in near real-time while ensuring that power 
systems meet their forecasted demands. The computational time for solving a probabilistic OPF formulation that minimizes both cost and risk has been significantly reduced when compared to the conventional classical enumeration method.

3) An AC-SCUC challenge has been successfully solved using an iterative successive linear programming (SLP) technique considering a fuzzy MILP formulation. The proposed method considers full AC transmission system model and constraints in the UC formulation. It also determines the optimal generation schedule even in the presence of transmission system outages, surpassing other more complex techniques in academic literature that use decomposition and linear approximation methods, which cannot account for transmission infeasibility. Further, the proposed method optimally schedules generation to minimize line flow violations where a violation free optimal solution is unavailable.

4) An MILP formulation for a fast security and risk constrained probabilistic unit commitment (SRCPUC) method with TAD model of WEGs is proposed that can optimally schedule generation to minimize costs and risk from WEG output uncertainty. The method models transmission system using DC model. The proposed method can be used to effectively manage large systems for a long period of planning horizon. These features cannot be accomplished by using the conventional classical enumeration technique. The SRCPUC algorithm is proposed and solved using the SLP technique. The SRCPUC algorithm also provides the unique and beneficial feature of computing the effect of standard deviation of wind energy forecast on the total schedule cost, which other methods cannot do. 
Overall, these algorithms and methods are very practical for real electricity market applications. Many ISOs, such as Independent System Operator (IESO) in Ontario, are seeking new methods to manage wind power.

\subsection{Recommendations}

A number of future directions for related research activities could be explored:

1) Revise the TAD model for other distribution models.

Many PDFs of distribution models are not symmetric, such as Weibull distribution. The TAD model cannot be used to approximate such distribution models. Some revisions are required to fit distribution models with asymmetric PDFs.

2) Seek miscellaneous stochastic representation of WEGs for long-term power system optimization.

The behaviors of wind power in a short-term are usually modeled using stochastic distribution functions. However, probability density functions cannot comprehensively represent wind power for long-term forecasts. Therefore, miscellaneous stochastic techniques, such as Chaos theory, could be explored to model wind power for long-term forecasts in power system operation.

3) Study economic aspects and market dynamics of current electricity markets to develop new framework for future electricity markets. 
Major electricity markets in North America and Europe are deregulated. Energy bidding systems and reserve markets are being developed, taking renewable energy into account as self-committed units. With increased consumption of wind energy, more economic aspects and market dynamics should be investigated for wind power scheduling.

4) Study the characteristics of smart grids to revise and update OPF/SCUC problem formulations to fit smart grids.

With more electric devices and machines such as electric vehicles and efficient energy storage units being designed and used in daily life, power system are facing a major shift from the traditional distribution system into a smart system with embedded intelligence and localized autonomy. New functions and features must eventually be integrated into the OPF/SCUC processes to fit such a new environment. 


\section{Appendix A}

\section{FUNDAMENTALS AND TECHNIQUES}

\section{A.1 Classical Enumeration Method}

In the classical enumeration method, WEGs are treated as a self-scheduled resource and all possible outputs of WEGs’ and corresponding EENS are computed offline.

Figure A.1 shows the normal probabilistic distribution (PDF) of one WEG. The dashed curve is the original probability density function created using the forecasted mean value of wind power, the bars represent 10 possible outputs of the WEG, and the dotted line is the probabilities of the ten power output values.

Combinations of all outputs of WEGs are taken to represent wind power scenarios in the system, as shown in Figure A.2. The EENS value of a scenario is computed by summing $E_{n}$ of every WEG. 


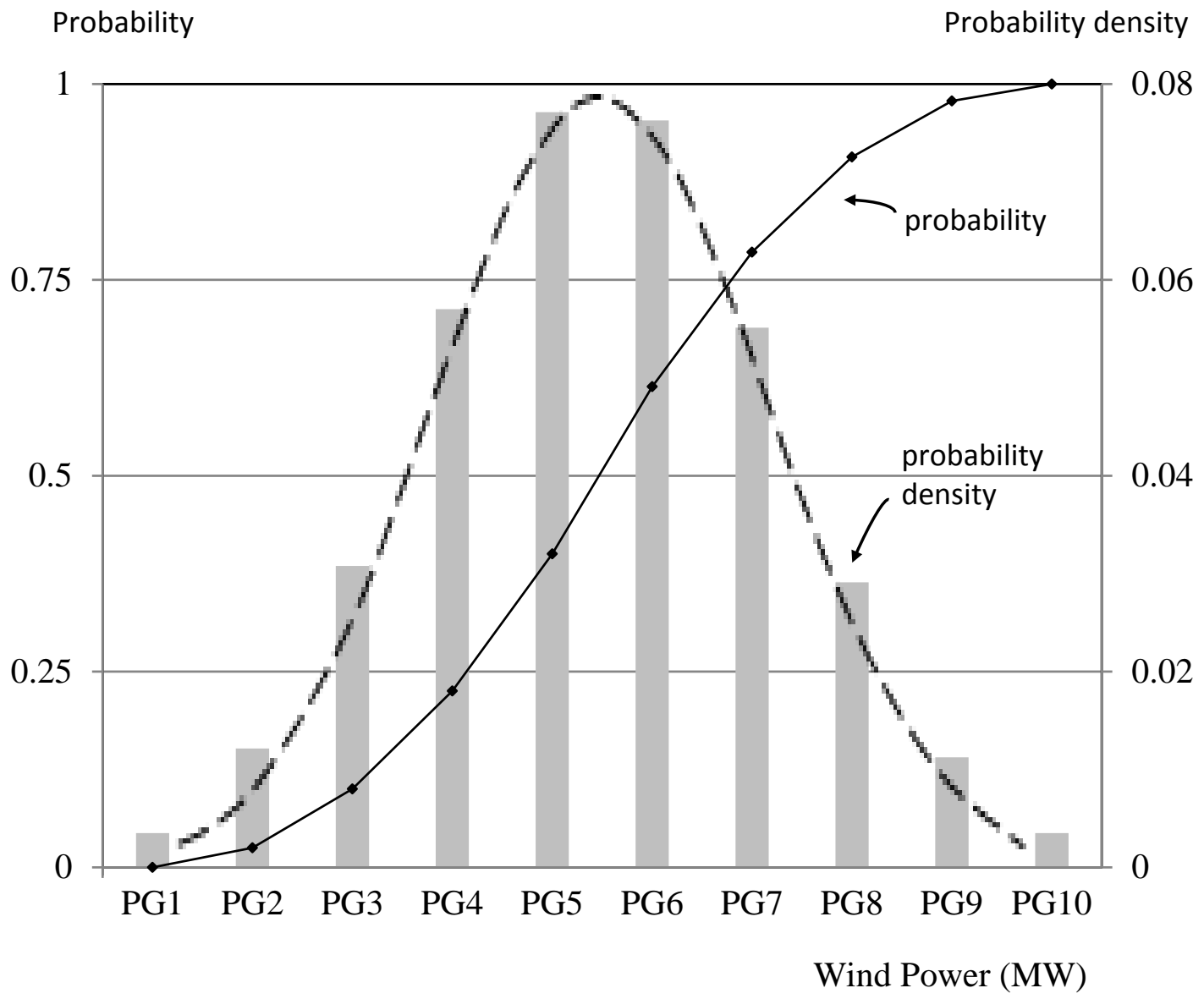

Fig. A.1 Probability density and cumulative distribution functions in classical enumeration method 


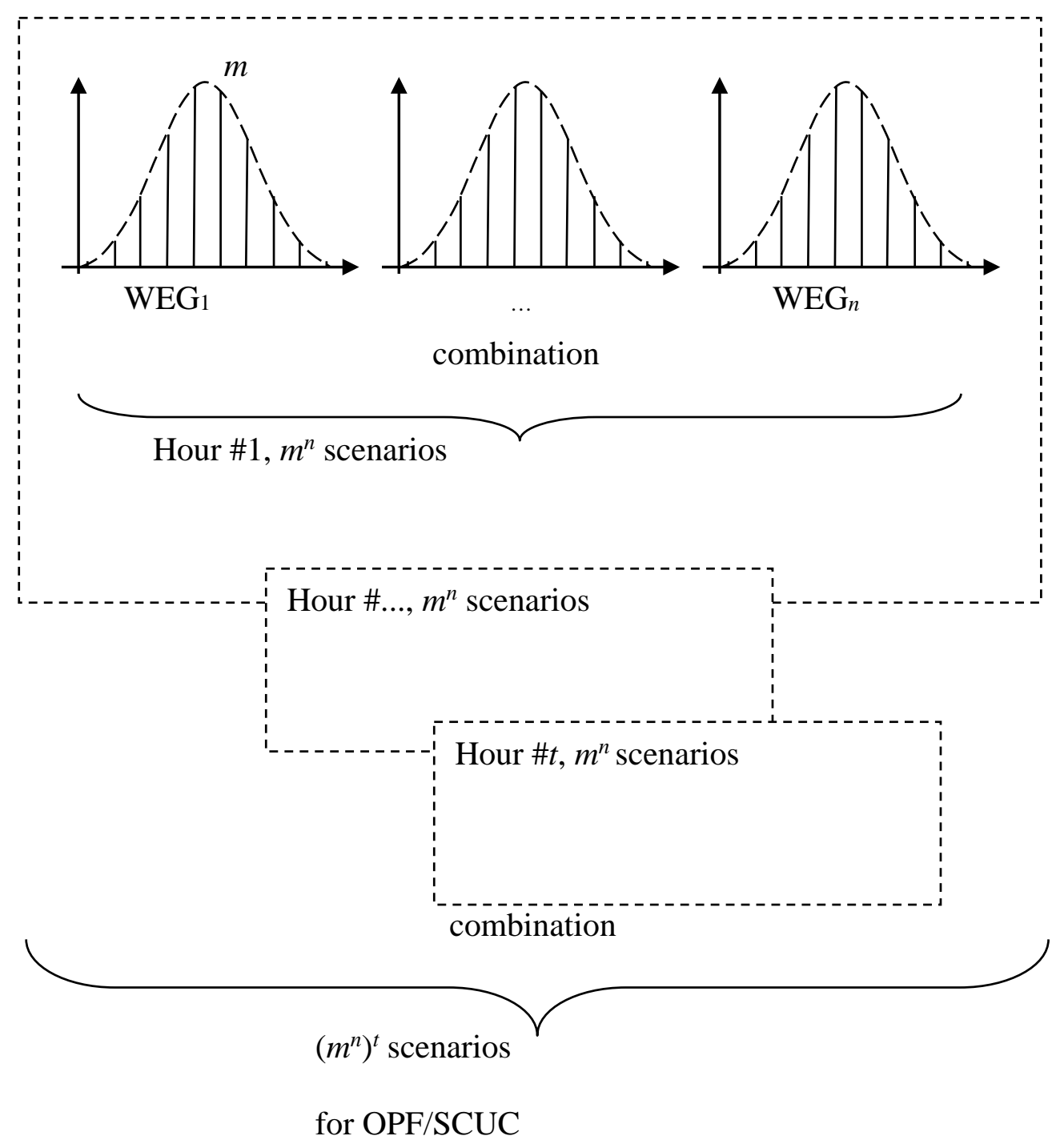

Fig. A.2 Scenarios production of power outputs of WEGs for OPF/SCUC process 
The OPF/SCUC is solved once for each scenario. In the example below, considering ' $n$ ' WEGs with ' $m$ ' possible outputs in ' $t$ ' hours ( $t=1$ for OPF), the number of scenarios equal $\left(m^{n}\right)^{t}$. Of these scenarios, OPF/SCUC is used to compute optimal schedules for each scenario. The optimal schedule with the least cost is chosen for the classical enumeration method. The algorithm of classical enumeration is shown in Figure A.3.

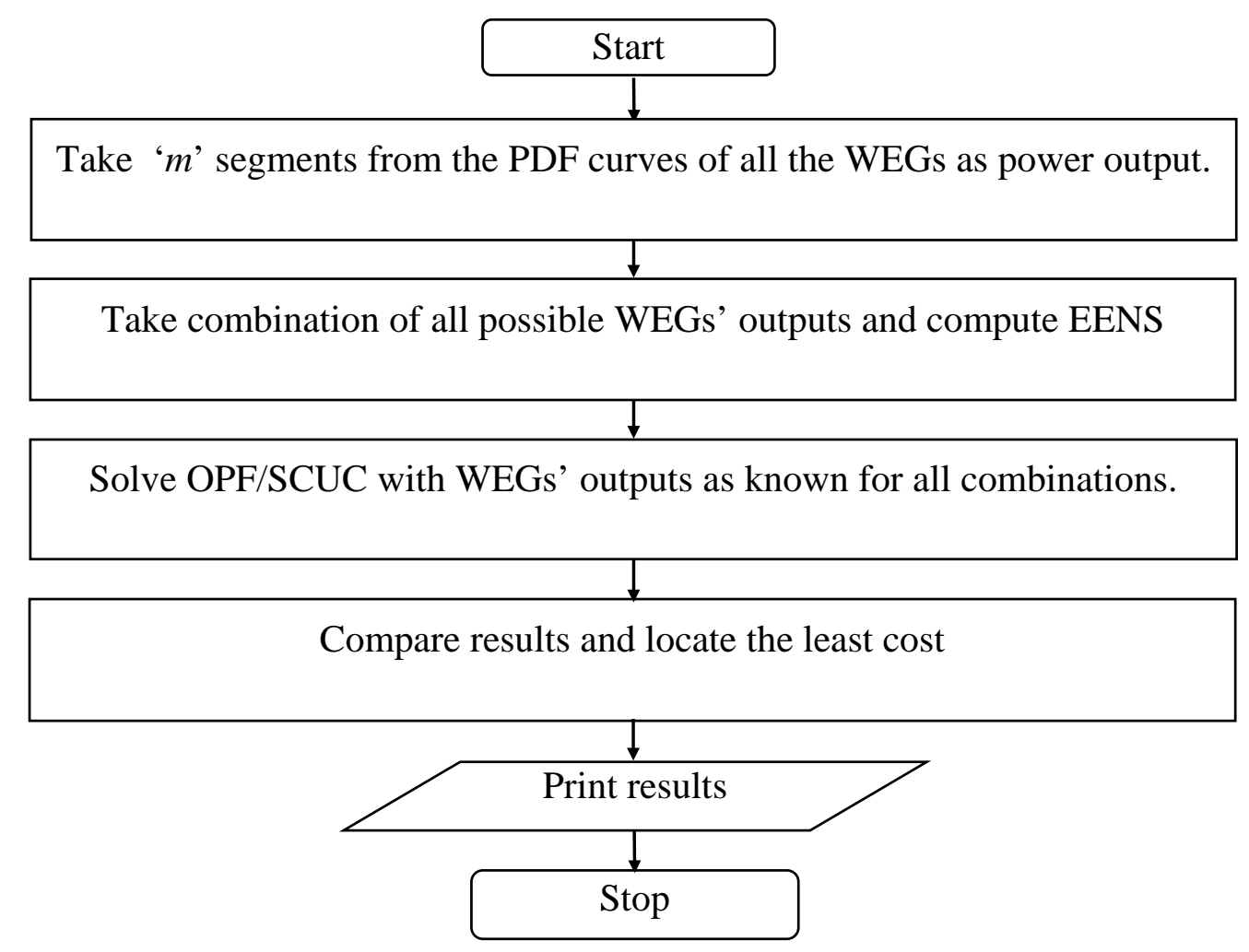

Fig. A.3 Flowchart of the classical enumeration 


\section{A.2 Power Balance Equations}

Transmission network constraints must be enforced in the OPF and UC problems to provide a network feasible solution while scheduling real power generation. Power balance equations, which are discussed as the following, are the basis for developing security constraints.

\section{A.2.1 AC Power Balance Equations}

Figure A.4 shows the net power injection of a practical bus in a power transmission system. The net power injection from the bus into the connected transmission lines, which is the function of bus voltage phase angles and magnitudes, must be equal to total power generation subtracted by the total demand on the bus. To solve these balance equations, reactive power generations, transmission line power flows, and bus voltage phase angles and magnitudes can be calculated.

$$
\begin{aligned}
& P T_{i}(V, \delta)=V_{i} \sum_{j}^{N B} V_{j \cdot} \cdot\left|Y_{i j}\right| \cdot \cos \left(\delta_{i}-\delta_{j}-\theta_{i j}\right) \forall i \\
& Q T_{i}(V, \delta)=V_{i} \sum_{j}^{N B} V_{j} \cdot\left|Y_{i j}\right| \cdot \sin \left(\delta_{i}-\delta_{j}-\theta_{i j}\right) \forall i
\end{aligned}
$$

In equations (A.1) and (A.2), $V$ is voltage magnitude of the $i^{\text {th }}$ bus, and $\delta$ is the corresponding voltage phase angle. $Y_{i j}$ is an element of the bus admittance matrix $Y$, and $\theta_{i j}$ is the phase angle of $Y_{i j}$. After solving the power balance equations, bus voltage and reactive power generation can be obtained. 


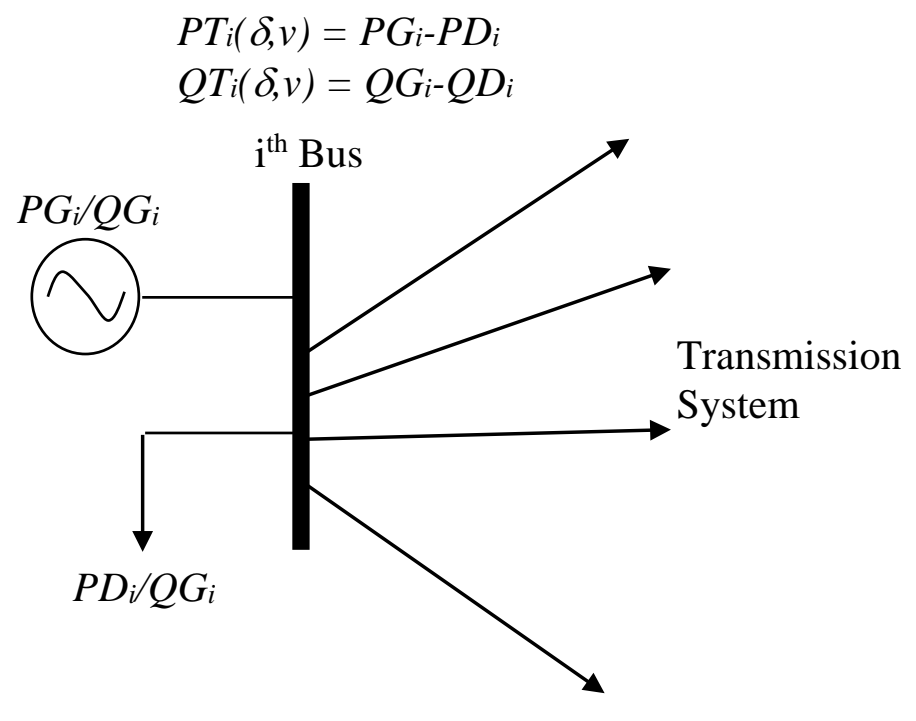

Fig. A.4 Net power injected into the transmission system by the $i^{\text {th }}$ bus

These power balance equations are called AC power balance equations and consider both real and reactive powers. These power balance equations can be solved by using load flow methods.

The load flow analysis, also called power flow, is a common computation technique used to analyze AC power balance equations [67]. Many methods, such as the Newton Raphson method, the fast decoupled method, and the Gauss Seidel method have been developed and successfully applied to solve power balance equations. A simplified method, derived from but also independent from AC power balance equations, is discussed as below. 


\section{A.2.2 DC Power Balance Equations}

DC power balance equations, solved by using the DC power flow method, are a simplified version of the AC Power balance equations. They are developed by assuming that all voltage magnitudes are constant and equal to a value of 1.0 per unit. Transmission line resistance, transmission losses, reactive power loads, and reactive power flows are completely ignored [67]-[69]. These assumptions are practically valid in transmission systems with large line reactance $(X)$ values and low line resistance $(R)$ values. With a high $X / R$ ratio in transmission systems, the real power flow mostly depends upon the bus voltage phase angles. With all of these assumptions, the real power balance equations can be simplified into linear functions of bus voltage angles and susceptance matrix.

$P T=B^{\prime} \cdot \delta$

In the (A.3), symbols $P$ and $\delta$ represent vectors of bus real power injections (excluding slack bus) and voltage phase angles (excluding slack bus). The slack bus phase angle is used as the reference, and is assumed to be zero. $B$ ' is the susceptance matrix in the bus frame of reference, with resistance in the transmission lines set to zero ohms and ignoring all shunt elements.

Although DC load flow is a less rigorous method and ignores voltage magnitude variations, it provides a very fast, linear, and direct calculation method in comparison with the full AC load flow method. The linear aspect of DC power balance equations is very computationally attractive, particularly when solving large-scale problems by employing linear optimization techniques. 


\section{A.3 Formulations of Transmission Security Constraints}

The transmission security constraints are derived from power balance equations. As mentioned in the previous section, the power balance equations can be categorized into AC/DC power balance equations. The general power balance equations are functions of bus voltage magnitudes and phase angles.

\section{A.3.1 AC Transmission Security Constraints}

The AC transmission security constraints are developed from the full AC power balance equations, which include both real and reactive power flows. The total power injection from a particular bus into a transmission system is a function of all bus voltage magnitudes and phase angles related to the bus. The power balance equations can be written in the following patterns.

Real power balance: $\quad P G_{i}-P D_{i}=P T_{i} \forall i$

Reactive power balance: $Q G_{i}-Q D_{i}=Q T_{i} \forall i$

Transmission line power flows can also be calculated using bus voltage magnitudes and phase angles with transmission line elements (line and shunt admittances). The $\pi$ model of transmission line is used to compute line power flows, as shown in Figure A.2.

The transmission line flow from bus $\mathrm{i}$ to bus $\mathrm{j}$ can be written as the following equations.

Line power flow:

$0 \leq S F_{l i} \leq \overline{S F_{l}} \forall l, i \in l$ 
$S F_{l i}=S L_{l i}\left(V_{i}, V_{j}, \delta_{i}, \delta_{j}\right)+S B_{l i}\left(V_{i}, \delta_{i}\right) \forall l, i \in l, j \in l$

Reactive power generation and bus voltage must be constrained within their bounds. In the day-ahead UC problem, reactive power has to be restricted by unit status (i.e., on-line or offline), which is an integer variable.

Reactive power generation limit:

For fast OPF: $\quad \underline{Q G_{n}} \leq Q G_{n} \leq \overline{Q G_{n}} \forall n \in C G$

For day-ahead UC: $U_{t n} \cdot Q G_{n} \leq Q G_{t n} \leq U_{t n} \cdot \overline{Q G_{n}} \forall t, n \in C G$

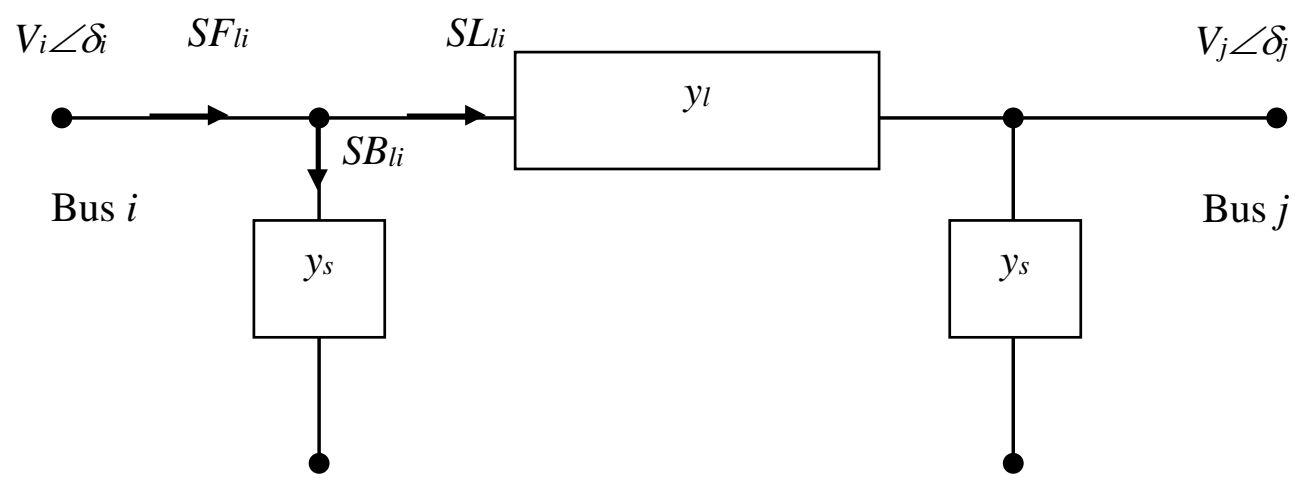

Fig. A.5 $\pi$ model of a transmission line between bus $i$ and $j$ 
Bus voltage limit:

$\underline{V_{i}} \leq V_{i} \leq \overline{V_{i}} \forall i$

$-\pi \leq \delta_{i} \leq \pi \forall i$

Compared to these security constraints, the DC transmission security constraint is a simplified version of the above equations. It is an approximation method to analyze the AC transmission system. Approximations are made for DC transmission security constraints, and the details are discussed in the next section.

\section{A.3.2 DC Transmission Security Constraints}

In a practical AC power transmission system, transmission line resistance is far smaller than line reactance. Thus, the line resistance can be ignored in system analysis. Voltage magnitudes are assumed to be at or close to unity p.u. for all buses, and reactive power generation is neglected.

The transmission line real power flow between bus $\mathrm{i}$ and bus $\mathrm{j}$ is limited within transmission line flow capacity and it can be calculated using the following equations:

$$
\begin{aligned}
& \frac{P L_{l}}{} \leq P L_{l} \leq \overline{P L_{l}} \quad \forall l \\
& P L_{l i}=\frac{\delta_{i}-\delta_{j}}{x_{i j}} \forall l, i \in l, j \in l
\end{aligned}
$$

By re-arranging equation (A.3), the voltage phase angle on buses can be computed by multiplying the inverse of B' to real power injection at buses. 
$\delta_{i}=\sum_{k} B I_{i k} \cdot P T_{k} \forall i$

$P L_{l i}=\frac{\delta_{i}-\delta_{j}}{x_{i j}}=\frac{1}{x_{i j}}\left(\sum_{k} B I_{i k} \cdot P T_{k}-\sum_{k} B I_{j k} \cdot P T_{k}\right)=\sum_{k}\left(\Theta_{l k} \cdot P T_{k}\right) \forall l, i \in l, j \in l$

The line power flow of DC transmission security constraints, equation (A.12), can be rewritten in terms of real power generation in equation (A.16).

$P L_{l i}=\sum_{k}\left[\Theta_{l k} \cdot\left(\sum_{n \in G I_{k}} P G_{n}-P D_{k}\right)\right] \forall l, i \in l, j \in l$

The coefficient matrix ' $\Theta$ ' is independent of real power output $\left(P G_{n}\right)$, and it can be calculated using the transmission line reactance and susceptance matrix. The equation (A.16) is an LP function of real power generation.

From equations (A.12) to (A.16), one may observe that the DC transmission security constraints are linear, and that they can be appropriately incorporated into an LP formulation and an MILP formulation. However, owing to simplification assumptions applied for the AC to DC conversion, the DC transmission security constraints could be less accurate than AC transmission security constraints. Therefore, it is important to consider the AC transmission security constraints in OPF and day-ahead UC.

\section{A.4 Successive Linear Programming}

The Successive Linear Programming (SLP) is a very efficient tool that has been used for the

OPF algorithm for several decades [62]. This tool was successfully applied in MILP 
formulations [63] [64], which are the bases of the UC problem in the current research work. Several variants of the SLP have been investigated in the literature [65]. The linear programming (LP) and MILP formulations are solved successively till optimality is achieved.

\section{A.5 Fuzzy Sets Theory}

Fuzzy sets theory is a mathematical technique that allows modeling of imprecise or conflicting engineering problems. In certain cases, impreciseness arises due to semantic uncertainty. In such problems, one commonly applies these processes: fuzzification, fuzzy rules/inference, and defuzzification. The fuzzy optimization procedure is another aspect of fuzzy sets theory. It provides a framework for handling optimization problems. It transforms the objective and the constraints into satisfaction functions of fuzzy sets.

The optimum result is achieved by maximizing the intersection of the satisfaction functions of the problem that are subject to other crisp constraints of the problem. Optimization of this formulation can be achieved by using an amenable optimization technique [66].

The AC transmission network constraints are complex in the MILP formulation. Getting a feasible solution is important for the SLP method. The use of fuzzy models of violating constraints is very useful for achieving a feasible solution. The fuzzy models allow for a relaxed enforcement of constraints, and as such they have been incorporated in the MILP solution technique to retain the robustness of the MILP technique [6]. 


\section{A.6 OPF used in Fuzzy MILP AC-SCUC}

The OPF algorithm has already been applied in the fuzzy MILP AC-SCUC problem in

Chapter 4. It is only performed when the power flow method fails to converge or violations occur on reactive power generation. The objective is to minimize the total change in real power generation of online generators in an hour:

Minimize: $\sum_{n \in G O N t}\left|\Delta P_{t n}\right| ; \forall t$

It is subject to:

(1) Power balance constraints,

(2) Voltage limits,

(3) Real power generation limits, and

(4) Reactive power generation limits. 


\section{APPENDIX B}

\section{DATA OF Test Systems}

\section{B.1Six-Bus System with One WEG}

The 6-bus system has already been tested in Chapter 4 and 5 for the SCUC problem. Figure B.1 shows the 6-bus system with one WEG. The diagram is used for the AC-SCUC problem in Chapter 4 by removing the WEG and increasing a multiple-connection line between Buses \#2 to \#4.

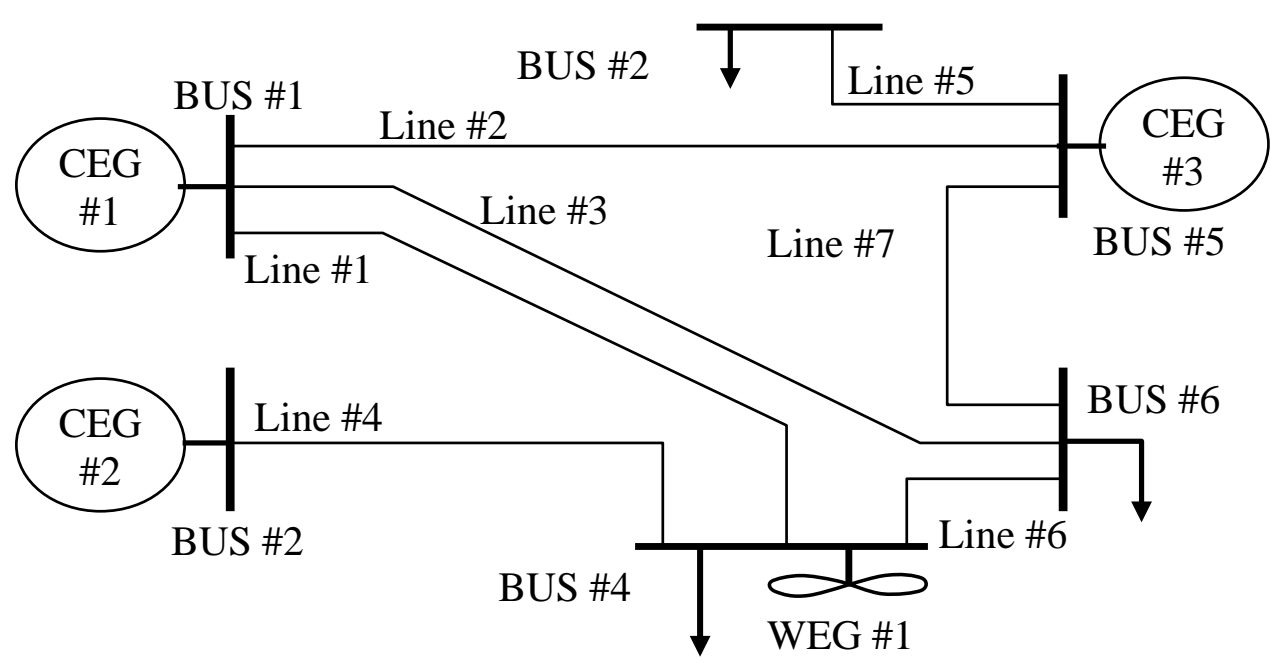

Fig. B.1 Six-bus Power System with one WEG 
Table B.1 CEG data of the 6-bus system

\begin{tabular}{|c|c|c|c|c|c|c|c|c|c|c|}
\hline Gen \# & Bus \# & $a c(\$)$ & $\begin{array}{c}b c 1 \\
(\$ / M W h)\end{array}$ & $\begin{array}{l}\overline{P G_{1}} \\
(\mathrm{MW})\end{array}$ & $\begin{array}{c}b c 2 \\
(\$ / \mathrm{MWh})\end{array}$ & $\begin{array}{l}\overline{P G_{2}} \\
(\mathrm{MW})\end{array}$ & $\begin{array}{c}b c 3 \\
(\$ / M W h)\end{array}$ & $\begin{array}{l}\overline{P G_{3}} \\
(\mathrm{MW})\end{array}$ & $\begin{array}{c}\underline{P G} \\
(\mathrm{MW})\end{array}$ & $\begin{array}{c}\overline{P G} \\
(\mathrm{MW})\end{array}$ \\
\hline 1 & 1 & 271.00 & 4.00 & 50 & 5.00 & 75 & 6.00 & 25 & 10 & 150 \\
\hline 2 & 2 & 132.00 & 8.50 & 50 & 9.50 & 100 & 11.00 & 750 & 10 & 900 \\
\hline 3 & 5 & 7.60 & 21.00 & 10 & 22.00 & 20 & 23.00 & 20 & 5 & 50 \\
\hline \multirow[t]{2}{*}{ Gen \# } & $s c$ & $r c$ & $U T$ & $D T$ & IC & $P 10$ & $R 10$ & $R 60$ & $\underline{Q_{n}}$ & $\overline{Q_{n}}$ \\
\hline & (\$/start) & (\$/MWh) & (hrs) & (hrs) & (hrs) & (MW) & $(\mathrm{MW})$ & (MW) & (Mvar) & (Mvar) \\
\hline 1 & 500.00 & 0.60 & 3 & 3 & 3 & 0 & 150 & 150 & -50 & 250 \\
\hline 2 & 300.00 & 0.11 & 3 & 2 & -2 & 0 & 900 & 900 & -50 & 140 \\
\hline 3 & 5.00 & 2.30 & 0 & 0 & -1 & 50 & 50 & 50 & -50 & 300 \\
\hline
\end{tabular}

Table B.2 WEG data of the 6-bus system

\begin{tabular}{|c|c|c|c|c|}
\hline \multirow{5}{*}{$\begin{array}{l}\# \\
\# \\
0 \\
1 \\
3\end{array}$} & Bus \# & $a w(\$)$ & $b w(\$ / M W h)$ & \\
\hline & 4 & 100 & 15 & \\
\hline & Forecasts & Hour \#1 & Hours \#2 & Hour \#3 \\
\hline & $\overline{\overline{P G}}(\mathrm{MW})$ & 80 & 100 & 60 \\
\hline & $\sigma(\mathrm{MW})$ & 8 & 10 & 6 \\
\hline
\end{tabular}


Table B.3 Load data of 6-bus system

\begin{tabular}{|c|c|c|c|c|c|c|c|}
\hline \multirow{2}{*}{$\begin{array}{l}\text { Load } \\
\qquad \#\end{array}$} & \multirow{2}{*}{$\begin{array}{c}\text { Bus } \\
\# \\
\#\end{array}$} & \multicolumn{2}{|c|}{ Hour \#1 } & \multicolumn{2}{|c|}{ Hours \#2 } & \multicolumn{2}{|c|}{ Hour \#3 } \\
\hline & & $\begin{array}{c}P D \\
\text { (MW) }\end{array}$ & $\begin{array}{c}Q D \\
\text { (Mvar) }\end{array}$ & $\begin{array}{c}P D \\
(\mathrm{MW})\end{array}$ & $\begin{array}{c}\text { QD } \\
\text { (Mvar) }\end{array}$ & $\begin{array}{c}P D \\
(\mathrm{MW})\end{array}$ & $\begin{array}{c}\text { QD } \\
\text { (Mvar) }\end{array}$ \\
\hline 1 & 4 & 71.43 & 50 & 85.72 & 60 & 78.57 & 55 \\
\hline 2 & 3 & 64.28 & 21.43 & 77.14 & 25.71 & 70.72 & 23.57 \\
\hline 3 & 6 & 114.29 & 78.57 & 137.14 & 94.29 & 125.71 & 85.43 \\
\hline
\end{tabular}

Table B.4 Network data of the 6-bus system

\begin{tabular}{c|c|c|c|c|c|c|c}
\hline Line \# & From Bus & To Bus & Number of lines & $R$ (p.u.) & $X$ (p.u.) & $B$ (p.u.) & MVA \\
\hline 1 & 1 & 4 & 1 & 0.035 & 0.225 & 0.013 & 300 \\
\hline 2 & 1 & 5 & 1 & 0.025 & 0.105 & 0.09 & 300 \\
\hline 3 & 1 & 6 & 1 & 0.04 & 0.215 & 0.011 & 300 \\
\hline 4 & 2 & 4 & 1 & 0 & 0.035 & 0 & $*$ \\
\hline 5 & 5 & 3 & 1 & 0 & 0.042 & 0 & 300 \\
\hline 6 & 4 & 6 & 1 & 0.028 & 0.125 & 0.007 & 300 \\
\hline 7 & 5 & 6 & 1 & 0.026 & 0.175 & 0.009 & 300 \\
\hline
\end{tabular}

* The capacity of transmission line between bus 2 and bus 4 is alternated for different test purposes. 


\section{B.2 IEEE 30-Bus System}

The detailed transmission system data can be found in [60]. The price data of CEG and WEG data are listed in the following tables. The system is tested for the OPF algorithm in Chapter 3.

Table B.5 CEG data of IEEE 30-Bus System

\begin{tabular}{|c|c|c|c|c|c|c|}
\hline Gen \# & $\begin{array}{c}b c 1 \\
\text { (\$/MWh) }\end{array}$ & $\begin{array}{l}\overline{P G}_{1} \\
(\mathrm{MW})\end{array}$ & $\begin{array}{c}b c 2 \\
(\$ / M W h)\end{array}$ & $\begin{array}{l}\overline{P G}_{2} \\
(\mathrm{MW})\end{array}$ & $\begin{array}{c}b c 3 \\
\text { (\$/MWh) }\end{array}$ & $\begin{array}{c}\overline{P G}_{3} \\
(\mathrm{MW})\end{array}$ \\
\hline G1 & 2.00 & 84 & 2.10 & 58 & 2.20 & 108 \\
\hline G2 & 3.00 & 50 & 3.20 & 50 & 3.40 & 50 \\
\hline G3 & 8.00 & 50 & 8.30 & 50 & 8.60 & 50 \\
\hline G4 & 10.00 & 34 & 10.40 & 8 & 10.80 & 33 \\
\hline G5 & 20.00 & 7 & 20.50 & 2 & 21.00 & 1 \\
\hline G6 & 50.00 & 7 & 50.60 & 2 & 51.20 & 1 \\
\hline Gen \# & $a c(\$)$ & $\begin{array}{c}\underline{P G} \\
\text { (MW) }\end{array}$ & $\begin{array}{c}\overline{P G} \\
(\mathrm{MW})\end{array}$ & $\begin{array}{c}\text { R10 } \\
\text { (MW) }\end{array}$ & $\begin{array}{c}\text { PGSTEP } \\
\text { (MW) }\end{array}$ & $\begin{array}{c}\text { VSTEP } \\
\text { (p.u.) }\end{array}$ \\
\hline G1 & 10.00 & 25 & 250 & 900 & 25 & 0.01 \\
\hline G2 & 10.00 & 25 & 150 & 900 & 15 & 0.01 \\
\hline G3 & 10.00 & 25 & 150 & 900 & 15 & 0.01 \\
\hline G4 & 10.00 & 25 & 75 & 900 & 10 & 0.01 \\
\hline G5 & 10.00 & 5 & 10 & 900 & 2 & 0.01 \\
\hline G6 & 10.00 & 5 & 10 & 900 & 2 & 0.01 \\
\hline
\end{tabular}


Table B.6 WEG Data of IEEE 30-Bus System

\begin{tabular}{c|c|c|c|c|c}
\hline Gen \# & $a w(\$)$ & $b w(\$ / M W h)$ & $\overline{\overline{P G}}(\mathrm{MW})$ & $\sigma$ & PGSTEP (MW) \\
\hline WEG1 & 10.00 & 5.00 & 20 & 2 & 2 \\
\hline WEG2 & 10.00 & 4.00 & 30 & 3 & 3 \\
\hline WEG3 & 10.00 & 2.50 & 50 & 5 & 5 \\
\hline
\end{tabular}

\section{B.3 IEEE 118-Bus System}

The transmission network and CEG data of modified IEEE 118-bus system are available in [46]. The costs of CEGs are linearized in Table B.9 to fit the LP/MILP formulation.

Table B.7 WEG Data of IEEE 118-Bus System in 3 hours

\begin{tabular}{|c|c|c|c|c|c|c|}
\hline Gen \# & Bus \# & $a w(\$)$ & $b w(\$ / M W h)$ & $\overline{\overline{P G}}(\mathrm{MW})$ & $\sigma$ & PGSTEP (MW) \\
\hline WEG1 & 10 & 80.00 & 5.00 & 300 & 30 & 30 \\
\hline WEG2 & 80 & 70.00 & 6.00 & 300 & 30 & 30 \\
\hline WEG3 & 89 & 70.00 & 7.00 & 300 & 30 & 30 \\
\hline
\end{tabular}


Table B.8 Forecasted output of WEGs in the 118-bus system (MW) in 24 hours

\begin{tabular}{|c|c|c|c|c|c|c|}
\hline \multirow{8}{*}{$\begin{array}{l}\# \\
\# \\
0 \\
\text { I1 } \\
3\end{array}$} & Hour \#1 & Hour \#2 & Hour \#3 & Hour \#4 & Hour \#5 & Hour \#6 \\
\hline & 313 & 269 & 312 & 292 & 318 & 284 \\
\hline & Hour \#7 & Hour \#8 & Hour \#9 & Hour \#10 & Hour \#11 & Hour \#12 \\
\hline & 280 & 292 & 309 & 302 & 350 & 285 \\
\hline & Hour \#13 & Hour \#14 & Hour \#15 & Hour \#16 & Hour \#17 & Hour \#18 \\
\hline & 292 & 281 & 320 & 343 & 288 & 273 \\
\hline & Hour \#19 & Hour \#20 & Hour \#21 & Hour \#22 & Hour \#23 & Hour \#24 \\
\hline & 298 & 268 & 344 & 260 & 340 & 306 \\
\hline \multirow{8}{*}{$\begin{array}{l}\text { \# } \\
0 \\
\text { I } \\
3\end{array}$} & Hour \#1 & Hour \#2 & Hour \#3 & Hour \#4 & Hour \#5 & Hour \#6 \\
\hline & 264 & 255 & 344 & 349 & 349 & 317 \\
\hline & Hour \#7 & Hour \#8 & Hour \#9 & Hour \#10 & Hour \#11 & Hour \#12 \\
\hline & 319 & 311 & 306 & 259 & 286 & 339 \\
\hline & Hour \#13 & Hour \#14 & Hour \#15 & Hour \#16 & Hour \#17 & Hour \#18 \\
\hline & 272 & 323 & 251 & 328 & 321 & 277 \\
\hline & Hour \#19 & Hour \#20 & Hour \#21 & Hour \#22 & Hour \#23 & Hour \#24 \\
\hline & 313 & 333 & 261 & 299 & 260 & 328 \\
\hline \multirow{8}{*}{$\begin{array}{l}\text { m } \\
0 \\
0 \\
1 \\
3\end{array}$} & Hour \#1 & Hour \#2 & Hour \#3 & Hour \#4 & Hour \#5 & Hour \#6 \\
\hline & 272 & 261 & 286 & 345 & 327 & 275 \\
\hline & Hour \#7 & Hour \#8 & Hour \#9 & Hour \#10 & Hour \#11 & Hour \#12 \\
\hline & 303 & 326 & 309 & 322 & 348 & 296 \\
\hline & Hour \#13 & Hour \#14 & Hour \#15 & Hour \#16 & Hour \#17 & Hour \#18 \\
\hline & 263 & 329 & 335 & 255 & 323 & 318 \\
\hline & Hour \#19 & Hour \#20 & Hour \#21 & Hour \#22 & Hour \#23 & Hour \#24 \\
\hline & 274 & 327 & 269 & 270 & 255 & 282 \\
\hline
\end{tabular}


Table B.9 CEG data of IEEE 118-Bus System

\begin{tabular}{|c|c|c|c|c|c|c|}
\hline Gen \# & $\begin{array}{c}b c 1 \\
(\$ / M W h)\end{array}$ & $\begin{array}{l}\overline{P G}_{1} \\
(\mathrm{MW})\end{array}$ & $\begin{array}{c}b c 2 \\
(\$ / \mathrm{MWh})\end{array}$ & $\begin{array}{l}\overline{P G}_{2} \\
(\mathrm{MW})\end{array}$ & $\begin{array}{c}b c 3 \\
(\$ / M W h)\end{array}$ & $\begin{array}{l}\overline{P G}_{3} \\
(\mathrm{MW})\end{array}$ \\
\hline G1 & 13.13 & 13.33 & 13.35 & 8.33 & 13.52 & 8.33 \\
\hline G2 & 12.13 & 13.33 & 12.35 & 8.33 & 12.52 & 8.33 \\
\hline G3 & 13.13 & 13.33 & 13.35 & 8.33 & 13.52 & 8.33 \\
\hline G4 & 10.67 & 266.67 & 14.50 & 116.67 & 16.83 & 116.67 \\
\hline G5 & 10.67 & 166.67 & 13.00 & 66.67 & 14.33 & 66.67 \\
\hline G6 & 12.17 & 16.67 & 12.40 & 6.67 & 12.53 & 6.67 \\
\hline G7 & 11.50 & 50.00 & 12.25 & 25.00 & 12.75 & 25.00 \\
\hline G8 & 12.13 & $\begin{array}{l}13.33 \\
\end{array}$ & 12.35 & 8.33 & 12.52 & 8.33 \\
\hline G9 & 13.13 & 13.33 & 13.35 & 8.33 & 13.52 & 8.33 \\
\hline G10 & 10.17 & 166.67 & 12.50 & 66.67 & 13.83 & 66.67 \\
\hline Gen \# & $a c(\$)$ & $\begin{array}{c}\underline{P G} \\
(\mathrm{MW})\end{array}$ & $\begin{array}{c}\overline{P G} \\
(\mathrm{MW})\end{array}$ & $\begin{array}{c}\text { R10 } \\
\text { (MW) }\end{array}$ & $\begin{array}{l}\text { PGSTEP } \\
\text { (MW) }\end{array}$ & $\begin{array}{l}\text { VSTEP } \\
\text { (p.u.) }\end{array}$ \\
\hline G1 & 130 & 5 & 30 & 30 & 5 & 0.005 \\
\hline G2 & 120 & 5 & 30 & 30 & 5 & 0.005 \\
\hline G3 & 130 & 5 & 30 & 30 & 5 & 0.005 \\
\hline G4 & 80 & 150 & 500 & 500 & 30 & 0.005 \\
\hline G5 & 90 & 100 & 300 & 300 & 30 & 0.005 \\
\hline G6 & 120 & 10 & 30 & 30 & 5 & 0.005 \\
\hline G7 & 110 & 25 & 100 & 100 & 17 & 0.005 \\
\hline G8 & 120 & 5 & 30 & 30 & 5 & 0.005 \\
\hline G9 & 130 & 5 & 30 & 30 & 5 & 0.005 \\
\hline G10 & 85 & 100 & 300 & 300 & 30 & 0.005 \\
\hline
\end{tabular}


Table B.9 CEG data of IEEE 118-Bus System (Cont')

\begin{tabular}{|c|c|c|c|c|c|c|}
\hline Gen \# & $\begin{array}{c}b c 1 \\
(\$ / M W h)\end{array}$ & $\begin{array}{l}\overline{P G}_{1} \\
(\mathrm{MW})\end{array}$ & $\begin{array}{c}b c 2 \\
(\$ / \mathrm{MWh})\end{array}$ & $\begin{array}{l}\overline{P G}_{2} \\
(\mathrm{MW})\end{array}$ & $\begin{array}{c}b c 3 \\
(\$ / M W h)\end{array}$ & $\begin{array}{l}\overline{P G}_{3} \\
(\mathrm{MW})\end{array}$ \\
\hline G11 & 9.83 & 183.33 & 12.50 & 83.33 & 14.17 & 83.33 \\
\hline G12 & 13.15 & 15.33 & $\begin{array}{l}13.38 \\
\end{array}$ & 7.33 & 13.53 & 7.33 \\
\hline G13 & 12.15 & 15.33 & 12.38 & 7.33 & 12.53 & 7.33 \\
\hline G14 & 11.50 & 50.00 & 12.25 & 25.00 & 12.75 & 25.00 \\
\hline G15 & 12.15 & 15.33 & 12.38 & 7.33 & 12.53 & 7.33 \\
\hline G16 & 11.50 & 50.00 & 12.25 & 25.00 & 12.75 & 25.00 \\
\hline G17 & 15.15 & 15.33 & $\begin{array}{l}15.38 \\
\end{array}$ & 7.33 & 15.53 & 7.33 \\
\hline G18 & 14.15 & 15.33 & 14.38 & 7.33 & 14.53 & 7.33 \\
\hline G19 & 12.50 & 50.00 & 13.25 & 25.00 & 13.75 & 25.00 \\
\hline G20 & 11.17 & 116.67 & 13.00 & 66.67 & 14.33 & 66.67 \\
\hline Gen \# & $a c(\$)$ & $\begin{array}{c}\underline{P G} \\
(\mathrm{MW})\end{array}$ & $\begin{array}{c}\overline{P G} \\
(\mathrm{MW})\end{array}$ & $\begin{array}{l}\text { R10 } \\
\text { (MW) }\end{array}$ & $\begin{array}{c}\text { PGSTEP } \\
\text { (MW) }\end{array}$ & $\begin{array}{l}\text { VSTEP } \\
\text { (p.u.) }\end{array}$ \\
\hline G11 & 80 & 100 & 350 & 350 & 30 & 0.005 \\
\hline G12 & 130 & 8 & 30 & 30 & 5 & 0.005 \\
\hline G13 & 120 & 8 & 30 & 30 & 5 & 0.005 \\
\hline G14 & 110 & 25 & 100 & 100 & 17 & 0.005 \\
\hline G15 & 120 & 8 & 30 & 30 & 5 & 0.005 \\
\hline G16 & 110 & 25 & 100 & 100 & 17 & 0.005 \\
\hline G17 & 150 & 8 & 30 & 30 & 5 & 0.005 \\
\hline G18 & 140 & 8 & 30 & 30 & 5 & 0.005 \\
\hline G19 & 120 & 25 & 100 & 100 & 17 & 0.005 \\
\hline G20 & 100 & 50 & 250 & 250 & 30 & 0.005 \\
\hline
\end{tabular}


Table B.9 CEG data of IEEE 118-Bus System (Cont')

\begin{tabular}{c|c|c|c|c|c|c}
\hline Gen \# & $b c 1$ & $\overline{P G}_{1}$ & $b c 2$ & $\overline{P G}_{2}$ & $b c 3$ & $\overline{P G}_{3}$ \\
\hline G21 & 12.17 & 116.67 & 14.00 & 66.67 & 15.33 & 66.67 \\
\hline G22 & 12.50 & 50.00 & 13.25 & 25.00 & 13.75 & 25.00 \\
\hline G23 & 13.50 & 50.00 & 14.25 & 25.00 & 14.75 & 25.00 \\
\hline G24 & 12.00 & 100.00 & 13.50 & 50.00 & 14.50 & 50.00 \\
\hline G25 & 12.00 & 100.00 & 13.50 & 50.00 & 14.50 & 50.00 \\
\hline G26 & 13.50 & 50.00 & 14.25 & 25.00 & 14.75 & 25.00 \\
\hline G27 & 11.07 & 206.67 & 14.20 & 106.67 & 16.33 & 106.67 \\
\hline G28 & 11.53 & 206.67 & 13.80 & 106.67 & 15.27 & 106.67 \\
\hline G29 & 8.57 & 153.33 & 11.70 & 73.33 & 13.83 & 73.33 \\
\hline G30 & 10.47 & 46.67 & 11.10 & 16.67 & 11.43 & 16.67 \\
\hline & & $\underline{P G}$ & $\overline{P G}$ & $R 10$ & PGSTEP & VSTEP \\
\hline Gen \# & $a c(\$)$ & & & & & \\
\hline G21 & 110 & 50 & 250 & 250 & 30 & 0.005 \\
\hline G22 & 120 & 25 & 100 & 100 & 17 & 0.005 \\
\hline G23 & 130 & 25 & 100 & 100 & 17 & 0.005 \\
\hline G24 & 110 & 50 & 200 & 200 & 30 & 0.005 \\
\hline G25 & 110 & 50 & 200 & 200 & 30 & 0.005 \\
\hline G26 & 130 & 25 & 100 & 100 & 17 & 0.005 \\
\hline G27 & 90 & 100 & 420 & 420 & 30 & 0.005 \\
\hline G28 & 100 & 100 & 420 & 420 & 30 & 0.005 \\
\hline G29 & 65 & 80 & 300 & 300 & 30 & 0.005 \\
\hline G30 & 100 & 30 & 80 & 80 & 14 & 0.005 \\
\hline & & & & & & \\
\hline & & $(\mathrm{MW})$ & $(\mathrm{MW})$ & $(\mathrm{MW})$ & $(\mathrm{MW})$ & $(\mathrm{p} . \mathrm{M})$. \\
\hline & & & & & \\
\hline & & & & & \\
\hline
\end{tabular}


Table B.9 CEG data of IEEE 118-Bus System (Cont')

\begin{tabular}{c|c|c|c|c|c|c}
\hline Gen \# & $b c 1$ & $\overline{P G}_{1}$ & $b c 2$ & $\overline{P G}_{2}$ & $b c 3$ & $\overline{P G}_{3}$ \\
& $(\$ / M W h)$ & $(\mathrm{MW})$ & $(\$ / \mathrm{MWh})$ & $(\mathrm{MW})$ & $(\$ / \mathrm{MWh})$ & $(\mathrm{MW})$ \\
\hline G31 & 11.17 & 16.67 & 11.40 & 6.67 & 11.53 & 6.67 \\
\hline G32 & 12.13 & 13.33 & 12.35 & 8.33 & 12.52 & 8.33 \\
\hline G33 & 11.10 & 10.00 & 11.25 & 5.00 & 11.35 & 5.00 \\
\hline G34 & 10.50 & 50.00 & 11.25 & 25.00 & 11.75 & 25.00 \\
\hline G35 & 9.50 & 50.00 & 10.25 & 25.00 & 10.75 & 25.00 \\
\hline G36 & 9.67 & 266.67 & 13.50 & 116.67 & 15.83 & 116.67 \\
\hline G37 & 10.50 & 50.00 & 11.25 & 25.00 & 11.75 & 25.00 \\
\hline G38 & 10.17 & 16.67 & 10.40 & 6.67 & 10.53 & 6.67 \\
\hline G39 & 12.50 & 350.00 & 17.50 & 150.00 & 20.50 & 150.00 \\
\hline G40 & 9.67 & 266.67 & 13.50 & 116.67 & 15.83 & 116.67 \\
\hline Gen \# & $a c(\$)$ & $\underline{P G}$ & $\overline{P G}$ & $R 10$ & PGSTEP & VSTEP \\
\hline G31 & 110 & 10 & 30 & 30 & 5 & 0.005 \\
\hline G32 & 120 & 5 & 30 & 30 & 5 & 0.005 \\
\hline G33 & 110 & 5 & 20 & 20 & 4 & 0.005 \\
\hline G34 & 100 & 25 & 100 & 100 & 17 & 0.005 \\
\hline G35 & 90 & 25 & 100 & 100 & 17 & 0.005 \\
\hline G36 & 70 & 150 & 500 & 500 & 30 & 0.005 \\
\hline G37 & 100 & 25 & 100 & 100 & 17 & 0.005 \\
\hline G38 & 100 & 10 & 30 & 30 & 5 & 0.005 \\
\hline G39 & 90 & 200 & 650 & 650 & 30 & 0.005 \\
\hline G40 & 70 & 150 & 500 & 500 & 30 & 0.005 \\
\hline & & & & & & \\
\hline & & $(\mathrm{MW})$ & $(\mathrm{MW})$ & $(\mathrm{MW})$ & $(\mathrm{MW})$ & $(\mathrm{p}$. u. $)$ \\
\hline
\end{tabular}


Table B.9 CEG data of IEEE 118-Bus System (Cont')

\begin{tabular}{|c|c|c|c|c|c|c|}
\hline Gen \# & $\begin{array}{c}b c 1 \\
(\$ / M W h)\end{array}$ & $\begin{array}{l}\overline{P G}_{1} \\
(\mathrm{MW})\end{array}$ & $\begin{array}{c}b c 2 \\
(\$ / \mathrm{MWh})\end{array}$ & $\begin{array}{l}\overline{P G}_{2} \\
(\mathrm{MW})\end{array}$ & $\begin{array}{c}b c 3 \\
(\$ / M W h)\end{array}$ & $\begin{array}{l}\overline{P G}_{3} \\
(\mathrm{MW})\end{array}$ \\
\hline G41 & 12.12 & 12.00 & 12.28 & 4.00 & 12.36 & 4.00 \\
\hline G42 & 12.30 & 30.00 & 12.70 & 10.00 & 12.90 & 10.00 \\
\hline G43 & 10.67 & 166.67 & 13.00 & 66.67 & 14.33 & 66.67 \\
\hline G44 & 13.67 & 166.67 & 16.00 & 66.67 & 17.33 & 66.67 \\
\hline G45 & 9.67 & 166.67 & 12.00 & 66.67 & 13.33 & 66.67 \\
\hline G46 & 13.12 & 12.00 & 13.28 & 4.00 & 13.36 & 4.00 \\
\hline G47 & 12.50 & 50.00 & 13.25 & 25.00 & 13.75 & 25.00 \\
\hline G48 & 11.50 & 50.00 & 12.25 & 25.00 & 12.75 & 25.00 \\
\hline G49 & 13.12 & 12.00 & 13.28 & 4.00 & 13.36 & 4.00 \\
\hline G50 & 12.33 & 33.33 & 12.75 & 8.33 & 12.92 & 8.33 \\
\hline Gen \# & $a c(\$)$ & $\begin{array}{c}\underline{P G} \\
(\mathrm{MW})\end{array}$ & $\begin{array}{c}\overline{P G} \\
(\mathrm{MW})\end{array}$ & $\begin{array}{c}\text { R10 } \\
\text { (MW) }\end{array}$ & $\begin{array}{c}\text { PGSTEP } \\
\text { (MW) }\end{array}$ & $\begin{array}{l}\text { VSTEP } \\
\text { (p.u.) }\end{array}$ \\
\hline G41 & 120 & 8 & 20 & 20 & 4 & 0.005 \\
\hline G42 & 120 & 20 & 50 & 50 & 9 & 0.005 \\
\hline G43 & 90 & 100 & 300 & 300 & 30 & 0.005 \\
\hline G44 & 120 & 100 & 300 & 300 & 30 & 0.005 \\
\hline G45 & 80 & 100 & 300 & 300 & 30 & 0.005 \\
\hline G46 & 130 & 8 & 20 & 20 & 4 & 0.005 \\
\hline G47 & 120 & 25 & 100 & 100 & 17 & 0.005 \\
\hline G48 & 110 & 25 & 100 & 100 & 17 & 0.005 \\
\hline G49 & 130 & 8 & 20 & 20 & 4 & 0.005 \\
\hline G50 & 120 & 25 & 50 & 50 & 9 & 0.005 \\
\hline
\end{tabular}


Table B.9 CEG data of IEEE 118-Bus System (Cont')

\begin{tabular}{c|c|c|c|c|c|c}
\hline Gen \# & $b c 1$ & $\overline{P G}_{1}$ & $b c 2$ & $\overline{P G}_{2}$ & $b c 3$ & $\overline{P G}_{3}$ \\
& $(\$ / M W h)$ & $(\mathrm{MW})$ & $(\$ / M W h)$ & $(\mathrm{MW})$ & $(\$ / \mathrm{MWh})$ & $(\mathrm{MW})$ \\
\hline G51 & 11.50 & 50.00 & 12.25 & 25.00 & 12.75 & 25.00 \\
\hline G52 & 11.50 & 50.00 & 12.25 & 25.00 & 12.75 & 25.00 \\
\hline G53 & 10.50 & 50.00 & 11.25 & 25.00 & 11.75 & 25.00 \\
\hline G54 & 10.33 & 33.33 & 10.75 & 8.33 & 10.92 & 8.33 \\
\hline Gen \# & $a c(\$)$ & $\underline{P G}$ & $\overline{P G}$ & $R 10$ & PGSTEP & VSTEP \\
\hline G51 & 110 & 25 & 100 & 100 & 17 & 0.005 \\
\hline G52 & 110 & 25 & 100 & 100 & 17 & 0.005 \\
\hline G53 & 100 & 25 & 100 & 100 & 17 & 0.005 \\
\hline G54 & 100 & 25 & 50 & 50 & 9 & 0.005 \\
\hline
\end{tabular}




\section{REFERENCE LIST}

[1] Renewable Energy Technology Roadmap 20\% by 2020. Retrieved from http://www.erec.org/fileadmin/erec_docs/Documents/Publications/Renewable_Energy_ Technology_Roadmap.pdf

[2] Status Update on Renewables Integration. Retrieved from http://www.ieso.ca/imoweb/pubs/consult/windpower/wpsc-20100923-RenewablesIntegration-update.pdf

[3] World Market Recovers and Sets A New Record: 42 GW of New Capacity in 2011, Total at 239 GW. Retrieved from http://www.wwindea.org/

[4] Park, G. L., Member, S., \& Schlueter, R. A., et al. (1985). A modified unit commitment and generation control for utilities with large wind generation penetrations. IEEE Transactions on Power Apparatus and Systems, PAS-104(7), 1630-1636.

[5] Foley, A. M., Leahy, P. G., \& Marvuglia, A., et al. (2012). Current methods and advances in forecasting of wind power generation. Renewable Energy, 37(1), 1-8.

[6] Venkatesh, B., Yu, P., \& Gooi, H. B., et al. (2008). Fuzzy MILP unit commitment incorporating wind generators. IEEE Transaction on Power Systems, 23(4), 1738-1746.

[7] Choling, D., Yu, P., \& Venkatesh, B. (2009). Effects of security constraints on unit commitment with wind generators. 2009 Power \& Energy Society General Meeting, IEEE, pp. 1-6, Jul. 2009. 
[8] Kamalinia, S., \& Shahidehpour, M. (2010). Generation expansion planning in windthermal power systems. IET Generation, Transmission \& Distribution, 4(8), 940-951.

[9] Dukpa, A., Duggal, I., \& Venkatesh, B., et al. (2010). Optimal participation and risk mitigation of wind generators in an electricity market. IET Renewable Power Generation, 4(2), 165-175.

[10] Yan, Y., Yang, S., \& Wen, F., et al. (2009). Generation scheduling with volatile wind power generation. 2009 International Conference on Sustainable Power Generation and Supply, pp. 1-7, Apr. 2009.

[11] Siahkali, H., \& Vakilian, M. (2010). Stochastic unit commitment of wind farms integrated in power system. Electric Power Systems Research, 80(9), 1006-1017.

[12] Wang, J., Botterud, A., \& Bessa, R., et al. (2011). Wind power forecasting uncertainty and unit commitment. Applied Energy, 88(11), 4014-4023.

[13] Normal distribution. Retrieved from https://en.wikipedia.org/wiki/Normal_distribution

[14] Camina, A., \& Lewis, B. (2011). An introduction to enumeration. New York, NY: Springer.

[15] Happ, H. H., \& Wirgau, K. A. (1981). A review of the optimal power flow. Journal of the Franklin Institute, 312(3/4), 231-264.

[16] Chowdhury, B. H., \& Rahman, S. (1990). A review of recent advances in economic dispatch. IEEE Transactions on Power Systems, 5(4), 1248-1259.

[17] Huneault, M., \& Galiana, F. D. (1991). A survey of the optimal power flow literature. IEEE Transactions on Power Systems, 6(2), 762-770. 
[18] Momoh, J. A., El-Hawary, M. E., \& Ramababu, A. (1999). A review of selected optimal power flow literature to 1993. I. Non-linear and quadratic programing approaches. IEEE Transactions on Power Systems, 14(1), 96-104.

[19] Unive-sity, H., El-Hawary, M. E., \& Adapa, R. (1999). A review of selected optimal power flow literature to 1993. II. Newton, linear programing and interior point methods. IEEE Transactions on Power Systems, 14(1), 105-111.

[20] Yamin, H. Y. (2004). Review on methods of generation scheduling in electric power systems. Electric Power Systems Research, 69(2-3), 227-248.

[21] Pandya, K. S., \& Joshi, S. K. (2008). A survey of optimal power flow methods. Journal of Theoretical and Applied Information Technology, 4(5), 450-458.

[22] Xia, X., \& Elaiw, A. M. (2009). Dynamic economic dispatch: A review. The Online Journal on Electronics and Electrical Engineering, 2(2), 234-245.

[23] Muthyala, M. B. M. S., \& Maheswarapu, S. (2010). Security constraint optimal power flow (SCOPF) - A comprehensive survey. International Journal of Computer Applications, 11(6), 42-52.

[24] Capitanescu, F., Martinez Ramos, J. L., \& Panciatici, P., et al. (2011). State-of-the-art, challenges, and future trends in security constrained optimal power flow. Electric Power Systems Research, 81(8), 1731-1741.

[25] Kumar, C., \& Alwarsamy, T. (2011). Dynamic economic dispatch - A review of solution methodologies. European Journal of Scientific Research, 64(4), 517-537.

[26] Dommel, H. W., \& Tinney, W. F. (1968). Optimal power flow solutions. IEEE Transactions on Power Apparatus and Systems, 87(10), 1866-1876. 
[27] Alsac, O., \& Stott, B. (1974). Optimal load flow with steady-state security. IEEE Transactions on Power Apparatus and Systems, PAS-93(3), 745-751.

[28] Stott, B., \& Marinho, J. L. (1979). Linear programming for power-system network security applications. IEEE Transactions on Power Apparatus and Systems, PAS-98(3), 837-848.

[29] Shao, W., \& Vittal, V. (2006). LP-based OPF for corrective FACTS control to relieve overloads and voltage violations. IEEE Transactions on Power Systems, 21(4), 18321839.

[30] Schweppe, F. C., Caramanis, M. C., \& Tabors, R. D., et al. (1988). Spot pricing of electricity. Norwell, MA: Kluwer.

[31] Sen, S., \& Kothari, D. (1998). Optimal thermal generating unit commitment: A review. Electrical Power \& Energy Systems, 20(7), 443-451.

[32] Yong, T., \& Lasseter, R. H. (2000). Stochastic Optimal Power Flow: Formulation and solution. IEEE Power-Engineering-Society Summer Meeting, 1, 237-242.

[33] Li, G., \& Zhang, X. P. (2011). Stochastic optimal power flow approach considering correlated probabilistic load and wind farm generation. IET Conference on Reliability of Transmission and Distribution Networks (RTDN 2011), Dexter House, London, UK, pp. 1-7, Nov. 2011.

[34] Madrigal, M., Ponnambalam, K., \& Quintana, V. H. (1998). Probabilistic optimal power flow. IEEE Canadian Conference on Electrical and Computer Engineering, Kitchener Waterloo, ON, Canada, vol. 1, pp. 385-388, May 1998. 
[35] Schellenberg, A., Rosehart, W., \& Aguado, J. (2005). Cumulant based Stochastic Optimal Power Flow (S-OPF) for variance optimization. 2005 IEEE Power Engineering Society General Meeting, San Francisco, CA, USA, vol. 1-3, pp. 473-478, Jun. 2005.

[36] Verbic, G., Schellenberg, A., \& Rosehart, W., et al. (2006). Probabilistic Optimal Power Flow Applications to Electricity Markets. IEEE 9th International Conference on Probabilistic Methods Applied to Power Systems, KTH, Stockholm, Sweden, vol. 1-2, pp. 348-353, Jun. 2006.

[37] Zhang, H., \& Li, P. (2010). Probabilistic analysis for Optimal Power Flow under uncertainty. IET Generation Transmission \& Distribution, 4, 553-561.

[38] Hu, Z., Wang, X., \& Taylor, G. (2010). Stochastic optimal reactive power dispatch: Formulation and solution method. International Journal of Electrical Power \& Energy Systems, 32(6), 615-621.

[39] Padhy, N. P. (2001). Unit commitment using hybrid models: A comparative study for dynamic programming, expert system, fuzzy system and genetic algorithms. Electrical Power \& Energy Systems, 23, 827-836.

[40] Padhy, N. P. (2004). Unit commitment - A bibliographical survey. IEEE Transactions on Power Systems, 19(2), 1196-1205.

[41] Salam, S. (2007). Unit commitment solution methods. World Academy of Science, Engineering and Technology, 35, 320-325.

[42] Farhat, I. A., \& El-Hawary, M. E. (2009). Optimization methods applied for solving the short-term hydrothermal coordination problem. Electric Power Systems Research, 79(9), 1308-1320. 
[43] Federal Energy Regulatory Commission. (2011). Recent ISO software enhancements and future software and modeling plans (staff report).

[44] Venkatesh, B., Jamtsho, T., \& Gooi, H. B. (2007). Unit commitment - A fuzzy mixed integer Linear Programming solution. IET Generation, Transmission \& Distribution, 1(5), 836-846.

[45] Guy, J. D. (1970). Security constrained unit commitment. IEEE Summer Power Meeting and EHV Conference, Los Angeles, CA, pp. 12-17, Jul. 1970.

[46] Fu, Y., Shahidehpour, M., \& Li, Z. Y. (2005). Security-constrained unit commitment with AC constraints. IEEE Transactions on Power Systems, 20(3), 1538-1550.

[47] Wang, J. H., Shahidehpour, M., \& Li, Z. Y. (2008). Security-constrained unit commitment with volatile wind power generation. IEEE Transaction on Power Systems, 23(3), 1319-1327.

[48] Fu, Y., \& Shahidehpour, M. (2007). Fast SCUC for large-scale power systems. IEEE Transaction on Power Systems, 22(4), 2144-2151.

[49] Wu, L., Shabidehpour, M., \& Li, T. (2007). Stochastic security-constrained unit commitment. IEEE Transaction on Power Systems, 22(2), 800-811.

[50] Fu, Y., Shahidehpour, M., \& Li, Z. Y. (2006). AC contingency dispatch based on security-constrained unit commitment. IEEE Transaction on Power Systems, 21(2), 897908.

[51] Martinez-Crespo, J., Usaola, J., \& Fernandez, J. L. (2006). Security-constrained optimal generation scheduling in large-scale power systems. IEEE Transaction on Power Systems, 21(1), 321-332. 
[52] Sifuentes, W. S., \& Vargas, A. (2007). Short-term hydrothermal coordination considering an AC network modeling. International Journal of Electrical Power and Energy Systems, 29(6), 488-496.

[53] Sifuentes, W. S., \& Vargas, A. (2007). Hydrothermal scheduling using Benders decomposition: Accelerating techniques. IEEE Transaction on Power Systems, 22(3), 1351-1359.

[54] Grey, A., \& Sekar, A. (2008). Unified solution of security-constrained unit commitment problem using a linear programming methodology. IET Generation. Transmission. \& Distribution, 2(6), 856-867.

[55] Bai, X., \& Wei, H. (2009). Semi-definite programming-based method for securityconstrained unit commitment with operational and optimal power flow constraints. IET Generation. Transmission. \& Distribution, 3(2), 182-197.

[56] Fu, Y., Shahidehpour, M., \& Li, Z. (2007). Security-constrained optimal coordination of generation and transmission maintenance outage scheduling. IEEE Transaction on power systems, 22(3), 1302-1313.

[57] Fu, Y., Li, Z., \& Shahidehpour, M., et al. (2009). Coordination of midterm outage scheduling with short-term security-constrained unit commitment. IEEE Transaction on power systems, 24(4), 1818-1830.

[58] Wu, L., Shahidehpour, M., \& Fu, Y. (2010). Security-constrained generation and transmission outage scheduling with uncertainties. IEEE Transaction on power systems, 25(3), 1674-1685. 
[59] Northeast Power Coordinating Council (NPCC) operating reserve criteria. Document A06. Retrieved from https://www.npcc.org/publicFiles/reliability/criteriaGuides Procedures/new/A-06.pdf

[60] IEEE 30-bus system. Retrieved from http://www.ee.washington.edu/research/pstca/pf30/pg_tca30bus.htm

[61] Green Energy Act. Retrieved from http://www.energy.gov.on.ca/en/green-energy-act/

[62] Pai, M. A., \& Paranjothi, S. R. (1975). Optimal power flow with security constraints using successive linear programming. IEEE PES Summer Meeting, Paper A 75 455-6, San Francisco, CA, Jul. 1975.

[63] Zhang, T., Elkasrawy, A., \& Venkatesh, B. (2009). A new computational method for reactive power market clearing. International Journal of Electrical Power and Energy Systems, 31(6), 285-293.

[64] Dukpa, A., Venkatesh, B., \& Chang, L. (2011). Fuzzy stochastic programming method: Capacitor planning in distribution systems with wind generators. IEEE Transactions on Power Systems, 26(4), 1971-1979.

[65] Venkatesh, B., Sadasivam, G., \& Khan, M. A. (2000). A new optimal reactive power scheduling method for loss minimization and voltage stability margin maximization using successive multi-objective fuzzy LP technique. IEEE Transactions on Power Systems, 15(2), 844-851.

[66] Zimmermann, H. J. (2001). Fuzzy set theory and its applications. Dordrecht, NL: Kluwer Academic Press.

[67] Glover, J. D., \& Sarma, M. S. (2008). Power system analysis and design (4th ed.). Toronto, ON: Thomson. 
[68] Overbye, T. J., \& Cheng, X., et al. (2004). A comparison of the AC and DC power flow models for LMP calculations. Proceedings of the 37th Annual International Conference on System Sciences, Jan. 2004.

[69] Purchala, K., Meeus, L., \& Van Dommelen, D., et al. (2005). Usefulness of DC power flow for active power flow analysis. 2005 Power Engineering Society General Meeting, IEEE, 1, 454-459.

[70]North American Electric Reliability Corporation (NERC). Reliability Concepts. Retrieved from http://www.nerc.com/files/concepts_v1.0.2.pdf. 